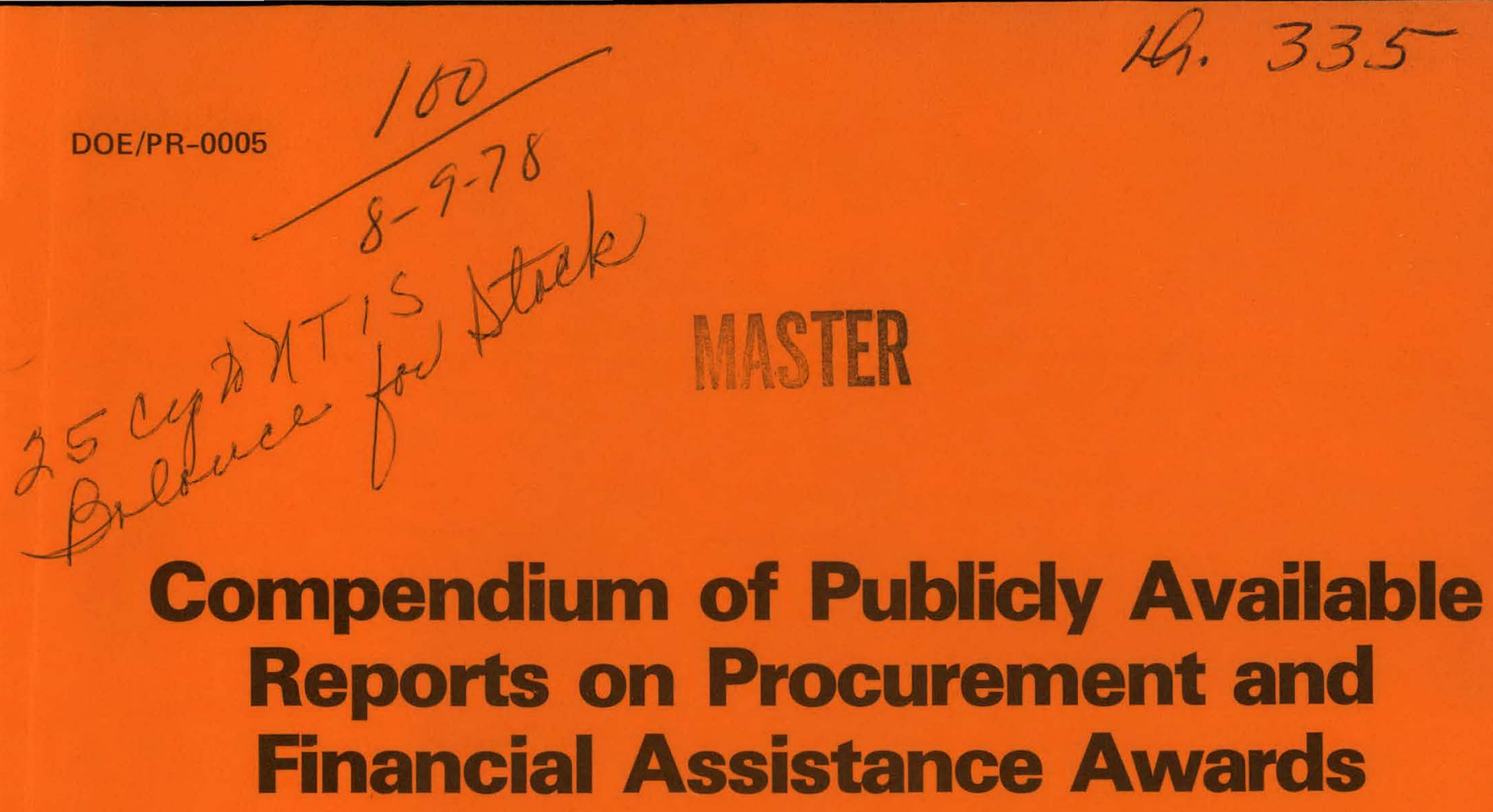

\title{
Compendium of Publicly Available Reports on Procurement and Financial Assistance Awards
}

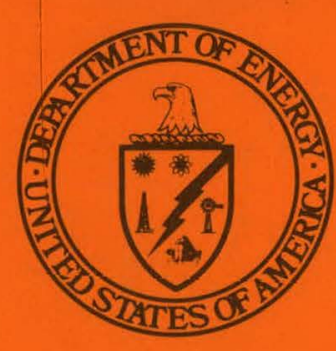

July 1978

\section{U.S. Department of Energy}

Directorate of Procurement and Contracts Management

Procurement Management Systems and Analysis Division 


\section{DISCLAIMER}

This report was prepared as an account of work sponsored by an agency of the United States Government. Neither the United States Government nor any agency Thereof, nor any of their employees, makes any warranty, express or implied, or assumes any legal liability or responsibility for the accuracy, completeness, or usefulness of any information, apparatus, product, or process disclosed, or represents that its use would not infringe privately owned rights. Reference herein to any specific commercial product, process, or service by trade name, trademark, manufacturer, or otherwise does not necessarily constitute or imply its endorsement, recommendation, or favoring by the United States Government or any agency thereof. The views and opinions of authors expressed herein do not necessarily state or reflect those of the United States Government or any agency thereof. 


\section{DISCLAIMER}

Portions of this document may be illegible in electronic image products. Images are produced from the best available original document. 
Available from:

National Technical Information Service (NTIS)

U.S. Department of Commerce

5285 Port Royal Road

Springfield, Virginia 22161

Price: Printed copy: $\$ 6.50$

Microfiche: $\$ 3.00$ 


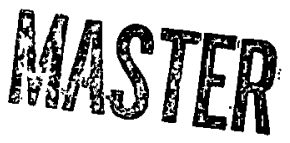

\section{Compendium of Publicly Available Reports on Procurement and Financial Assistance Awards}

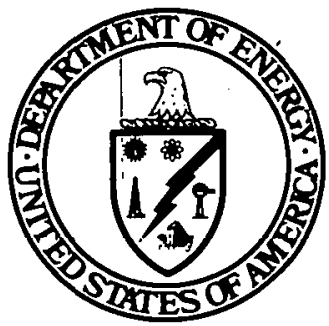

July 1978
This NOTICE

This report was prepared as an account of work sponsored by the United States Government. Neither the United States not the United States Department of

Energy, nor any of their employees, nor any of their

contractors, subcontractors, or their employees, makes

any warranty, express or implied, or assumes any lesal

liability or responsibility for the accuracy, completeness

or usefulness of any information.

process disclosed, or represents that its use would ar

process disclosed, or represents that its use would not
infringe privately owned rights.

\section{U.S. Department of Energy}

Directorate of Procurement and Contracts Management

Procurement Management Systems and Analysis Division

Washington, D.C. 20545 
Foreword .............Page $V$

I. Introduction..........Page VII

II. Published Reports......Pages $1-37$

A. United States Department of Energy

Summary of Total Department Prccurement and Financial Assistance Obligations by Federal Region

B. United States Department of Energy Summary of Department Procurement and Financial Assistance Obligations to Government Entities by Federal Region

C. United States Department of Energy Summary of Department Procurement and Financial Assistance Cbligations to Non-Government Entities by Federal Region

D. United States Department of Energy Summary of Total Procurement Awards

by Federal Region--Prime

E. United States Department of Energy Summary of Procurement Awards to Government Entities by Federal Region--Prime

F. United States Department of Energy Summary of Procurement Awards to Non-Government Entities by Federal Region--Frime

G. Unitea States Department of Energy Summary of Total Financial Assistance Awards by Feoieral Region

H. Linited States Department of Energy Summary of Financial Assistance Awards to Government Entities by Federal Region

I. United States Department of Energy Summary of Financial Assistance Awards to Non-Government Entities by Federal Region 
J. United States Department of Energy Summary of Total Department. Procurement and Financial Assistance Obligations by State

K. United States Department of Energy Summary of Department.Procurement and Financial Assistance Obligations to Government Entities by State

L. United States Department of Energy Summary of Department Procurement and Financial Assistance Obligations. to Non-Government Entities by State

M. United States Department of Energy Summary of Total Frocurement Awards

by State--Prime

N. United States Department of Energy Summary of Procurement Awards to Government.

Ent1ties by state--Prime.

O. United States Department of Energy Summary of Procurement Awards to Non-Government Entities by State--Prime

P. United States Department of Energy Summary of Total Financial Assistance Awards by State

Q. United States Department of Energy Summary of Financial Assistance Awards to Government. Entities by State

R. United States Department of Energy Summary of Financial Assistance Awards to Non-Government Entities by State

III. Unpublished Reports ........Pages 38-67

A. Active Prime Contracts by State, by Program

B. Active Prime Contracts by Program, by State

C. Active Prime Contracts--Alphabetical

D. Active Prime Contracts by Type of Work 
E. Active Prime Operating Contracts

F. Active DOE Grants

G. Quick-Look Index--Alphabetical

H. Quick-Look Index--Numeric

I. Alphabetical Index

J. Type of Contract Index

K. Type of Work Index

L. Statistics Report

M. Contractor Ranking by Current FY Obligations

N. Summary of Top 101 Contractors

IV. Special Reports .....Page 69

V. Acting Regional Representatives of the Secretary..Page 71

VI. Energy Extension Services Offices........Pages 73-74

VII. Appendix ............Pages 75-end 


\section{THIS PAGE}

\section{WAS INTENTIONALLY LEFT BLANK}


Energy is a dynamic, innovative and challenging field, which encompasses everyone--users, producers, researchers and investors. Therefore, there is a growing public interest in energy. The private sector is deeply involved in energy and has responsibilities which parallel those of the Federal Government.

The public has shown a particular interest in the Department of Energy's procurement, contracting and financial assistance programs. Procurement systems reports involve extensive information on over 6,000 active prime contracts, grants, loan guarantees and agreements of several types; plus over 30,000 small purchase transactions.

In their quest for information, the public, Congress and DOE personnel experience the frustration of wending their way through bureaucratic mazes, countless people and phone numbers as well as letter and memo exchanges. Hopefully, this Compendium will eliminate some confusion and will serve the needs of most of the people who wonder what kind of procurement systems reports are available from the Department of Energy. Since our systems are undergoing continual enhancement, this publication will be revised periodically. 


\section{THIS PAGE}

\section{WAS INTENTIONALLY \\ LEFT BLANK}


This Compenaium has three major sections.

Published Reports--This section includes computergenerated reports, which will be published annually with a cumulative monthly update. The annual reports will be published as of september 30 , the end of the Federal Fiscal Year. Alongside each report is a summary of the report showing the data elements included in the report.

When available, these reports can be obtained from your nearest Energy Extension Service Office. A list of the Department of Energy's ten Energy Extension Service offices is Included in this publication.

Unpublished Reports--This section is comprised of available, computer-generated reports with a summary of each report and a listing of data elements included in the report. Unpublished reports are reformats or subsets of information available in the published reports and are generated on a limited basis for repetitive Departmental needs. They are available at $\$ 0.10$ per page. (No charge is made for requests of ten pages or less.)

Requests for unpublished reports should be addressed to:

\author{
Reports and Analysis Branch \\ Procurement Management Systems \\ and Analysis Division \\ Procurement and Contracts \\ Management Directorate \\ U.S. Department of Energy \\ Washington, D.C. 20545
}

Special Reports--Special report formats or subsets of this information can be obtained by writing to the same above address. In addition to the per page costs, setup and computer costs are also charged the requester.

One man is available part-time to fill all requests. Therefore, responses to requests for unpublished, available reports require two to three weeks for delivery. However, if special programming is necessary, delivery may take six weeks to three months. 
II. Published Reports

1 
UNITED STATES DEPARTMENT OF ENERGY

SUMMARY OF TOTAL DEPARTMENT PROCUREMENT AND FINANCIAL ASSISTANCE

OBLIGATIONS BY FEDERAL REGION

This report incluces the following data for each region and summarized for all regions, U.S. Possessions, foreign countries and all of these:

- Total Active Awards (1)

- Current Fiscal Year's Obligations (2)

- Prior Fiscal Year's Obligations (3)

- Earlier Fiscal Years' Obligations (4)

- Total Obligations to date (5)

- Face Value of Awards (6) 


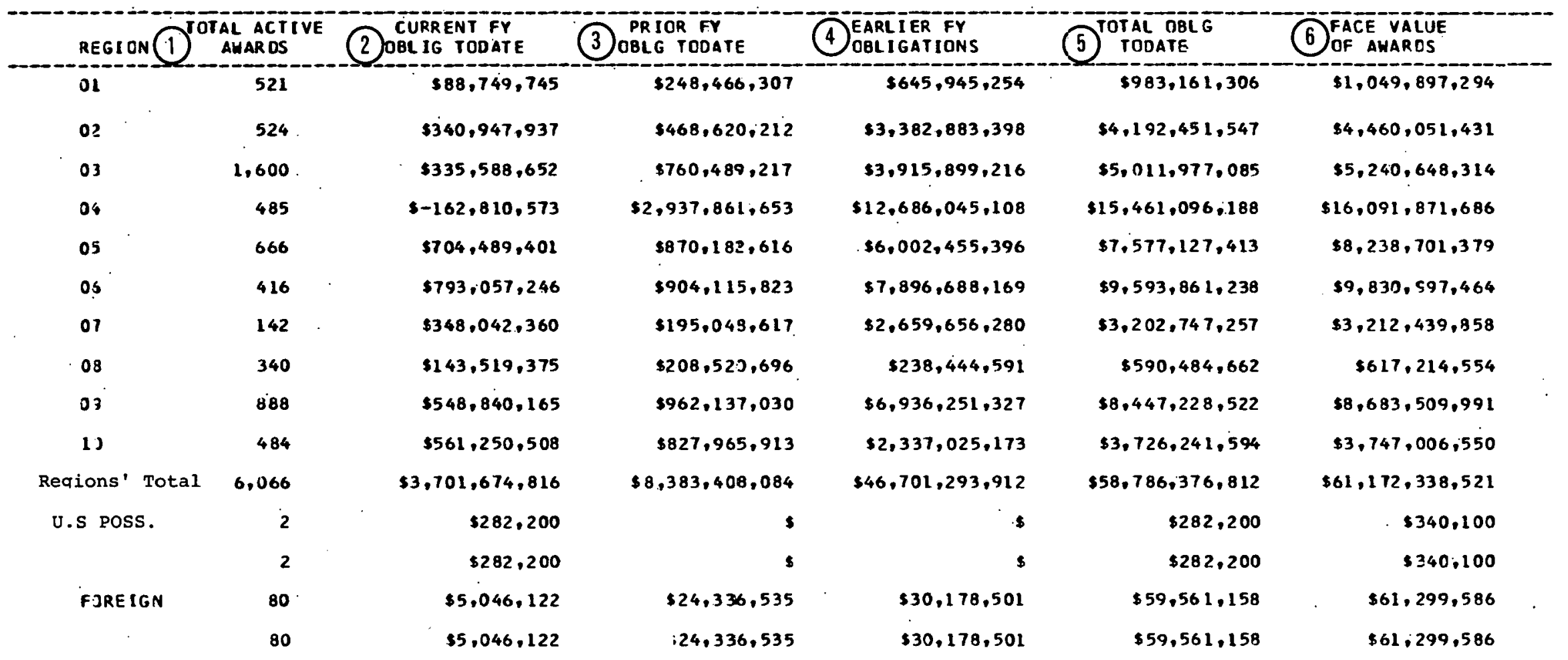


UNITED STATES DEPARTMENT OF ENERGY
SUMMARY OF DEPARTMENT PROCUREMENT AND FINANCIAL ASSISTANCE
OBLIGATIONS TO GOVERNMENT ENTITIES BY FEDERAL REGION

This report includes the following data for each region and summarized for all regions, U.S. Possessions, foreign countries and all of these:

- Total Active Awards (1)

- Current Fiscal Year's Obligations (2)

- Prior Fiscal Year's Obligations (3)

- Earlier Fiscal Years' Obligations (4)

- Total Obligations to date (5)

- Face Value of Awards (6) 
IPROGRAM NUMBER - M0402651)

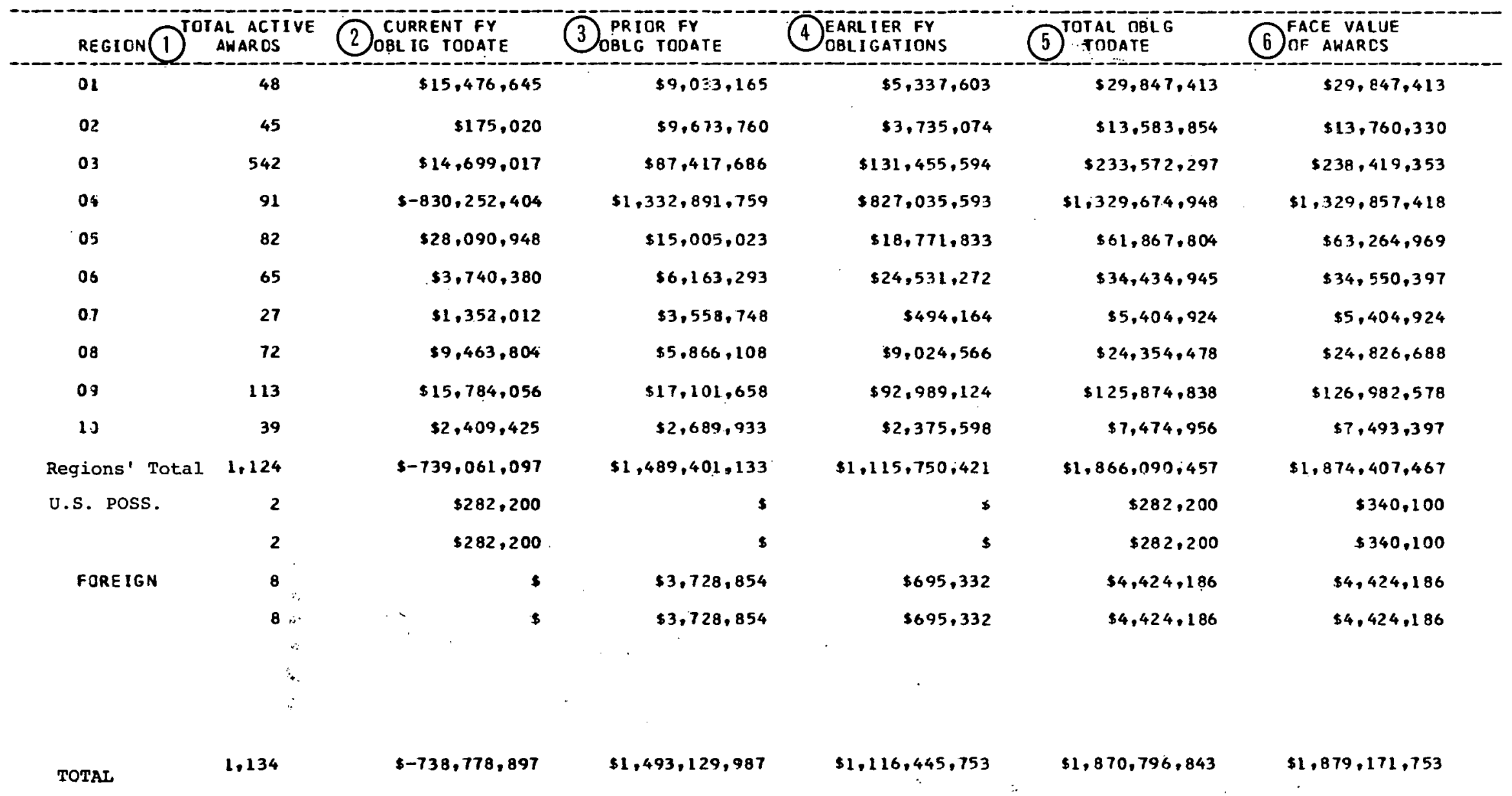


UNITEE STATES DEPARTMENT OF ENERGY
SUMMARY OF DEPARTMENT PROCUREMENT AND FINANCIAL ASSISTANCE
OBLIGATIONS TO NON-GOVERNMENT ENTITIES BY FEDERAL REGION

This report includes the following data for each region and summarized for all regions, U.S. Possessions, foreign countries and all of these:

- Total Active Awards (1)

- Current Fiscal Year's Obligations (2)

- Prior Fiscal Year's Obligations (3)

- Earlier Fiscal Years' Obligations (4)

- Total Obligations to date (5)

- Face Value of Awards (6) 
UNI TED STATES DEPARTMENT OF ENERGY
SUMMARY OF TOTAL DEPARTMENT PROCUREMENT AND FINANCIAL ASSISTANCE

OBL IGATIONS TO NON-GOVERNMENT ENTITIES BY FEDERAL REGION

PROGRAM NUMBER - M04026511

\begin{tabular}{|c|c|c|c|c|c|c|}
\hline REGICN 1$)^{\operatorname{To}}$ & $\begin{array}{l}\text { AL ACT I } \\
\text { AHARDS }\end{array}$ & $\begin{array}{l}\text { CURRENT FY } \\
\text { OBLIG TCOATE }\end{array}$ & $\begin{array}{l}\text { PRIOR FY } \\
\text { OBLG TOCATE }\end{array}$ & $\begin{array}{l}\text { EARLIER FY } \\
\text { GBLIGATIONS }\end{array}$ & $5)^{\text {TOTAL OBLG }}$ & $\begin{array}{l}\text { FACE VALUE } \\
\text { OF AWARDS }\end{array}$ \\
\hline 01 & 470 & $\$ 73,273,100$ & $\$ 238,961,299$ & $\$ 640,470,151$ & $\$ 952,704,550$ & $\$ 1,019,435,721$ \\
\hline 02 & 476 & $\$ 340.772 .917$ & $\$ 458,946,452$ & $\$ 3,378,401,208$ & $\$ 4,178,120,577$ & $\$ 4,445,543, \$ 85$ \\
\hline 03 & 1.043 & $\$ 320,837,135$ & $\$ 672,438.707$ & $\$ 3,783,547,803$ & $\$ 4,776,823,645$ & $\$ 5.000 .572,433$ \\
\hline 04 & 387 & $\$ 671.383 .038$ & $\$ 1,614,148,691$ & $\$ 11,803,020,185$ & $\$ 14,088,551,914$ & $\$ 14,719,144,942$ \\
\hline 05 & 574 & $\$ 676,387,953$ & $\$ 855,159,593$ & $55,974,095,239$ & $\$ 7,505,642,785$ & $\$ 8,165,819,586$ \\
\hline 06 & 351 & $\$ 789,316,866$ & $\$ 897,952.530$ & $\$ 7,872,156,897$ & $\$ 9.559,426.293$ & $\$ 9.796 .447 .067$ \\
\hline 07 & {$[11$} & $\$ 346,690,348$ & $\$ 191.482 .394$ & $\$ 2,658,953,998$ & $\$ 3,197,126,740$ & $\$ 3,206,808,598$ \\
\hline 08 & 266 & $\$ 134,055,571$ & $\$ 202,533,369$ & $\$ 228,965,909$ & $5565.554,849$ & $\$ 591,802,637$ \\
\hline 09 & 768 & $\$ 532.758 .746$ & $\$ 944,896,677$ & $.56,842,317,877$ & $\$ 8,319,973,300$ & . $\$ 8,555,067,613$ \\
\hline 10 & 443 & $\$ 558,784,405$ & $\$ 825.275 .980$ & $\$ 2.334 .649 .575$ & $\$ 3,718,709,960$ & $\$ 3,739,456,475$ \\
\hline $\begin{array}{l}\text { Regions' } \\
\text { Total }\end{array}$ & 4,887 & $\$ 4.444 .260 .079$ & $\$ 6,901,795,692$ & $\$ 45,516,578,842$ & $\$ 56,862,634,613$ & $\$ 59,240,099.457$ \\
\hline \multirow[t]{2}{*}{ FOREIGN } & 57 & $\$ 4.968 .669$ & $\$ 20.231,020$ & $\$ 27.796 .730$ & $\$ 52,996,419$ & $\$ 54,734,847$ \\
\hline & 57 & $\$ 4,968,669$ & $\$ 20.231 .020$ & $\$ 27,796,730$ & $\$ 52,996,419$ & $\$ 54,734,847$ \\
\hline
\end{tabular}

TOTAL 
UNITED STATES DEPARTMENT OF ENERGY

SUMMARY OF TOTAL PROCUREMENT AWARDS

BY FEDERAL REGION--PRIME

This report includes the following data for each region and summarized for all regions, U.S. Possessions, foreign countries and all of these:

- Total Active Awards (1)

- Current Fiscal Year's Obligations (2)

- Prior Fiscal Year's Obligations (3)

- Earlier Fiscal Years' Obligations (4)

- Total obligations to date (5)

- Face Value of Awards (6) 
(PRDGRAM NUMBER - M0402651)

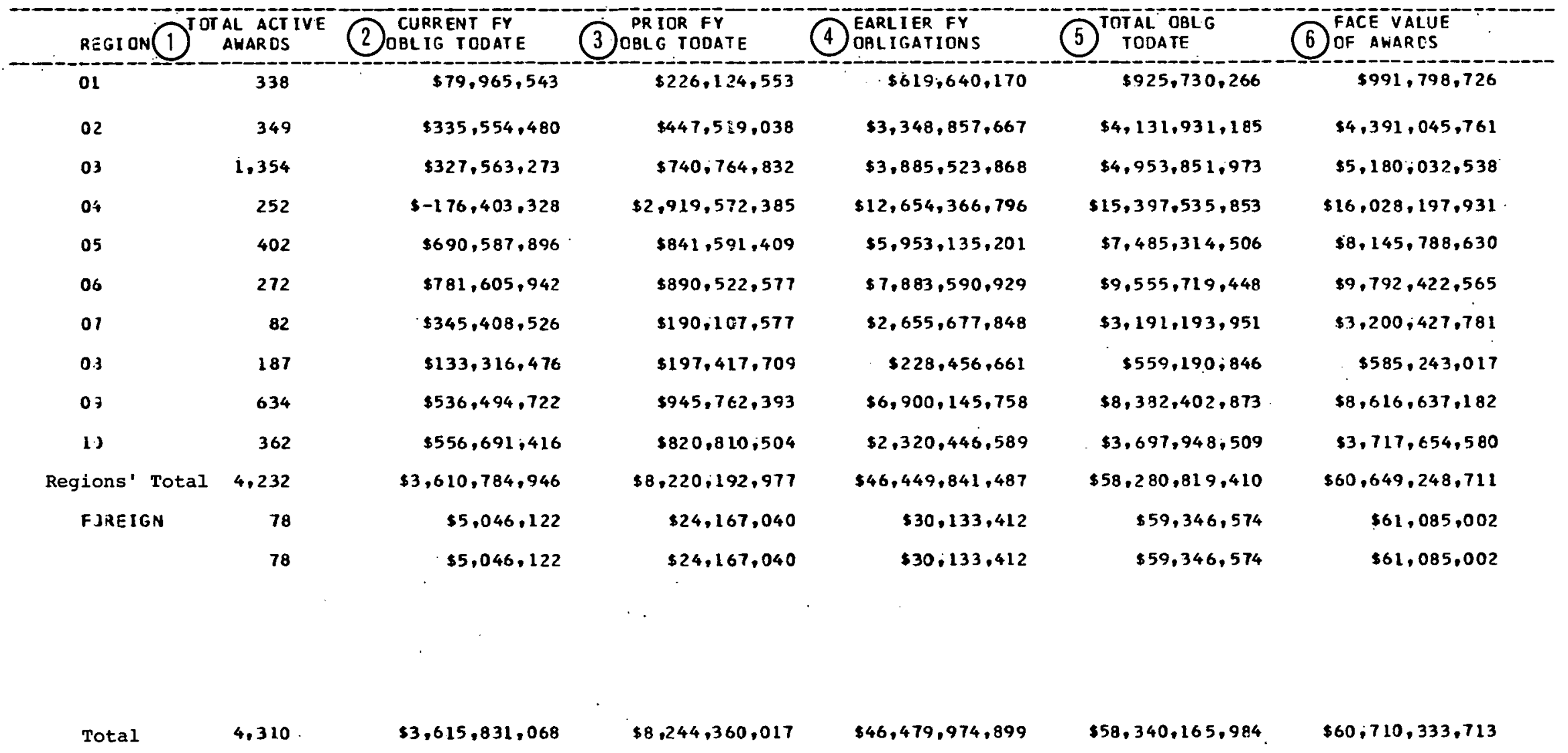




\section{UNITED STATES DEPARTMENT OF ENERGY}

SUMMARY OF PROCUREMENT AWP.RDS

TO GOVERNMENT ENTITIES BY FEDERAL REGION--PRIME

This report includes the following data for each region and summarized for all regions, U.S. Possessions, foreign countries and all of these:

- Total Active Awards (1)

- Current Fiscal Year's Obligations (2)

- Prior Fiscal Year's Obligations (3)

- Earlier Fiscal Years' Obligations (4)

- Total Obligations to date (5)

- Face Value of Awards (6) 


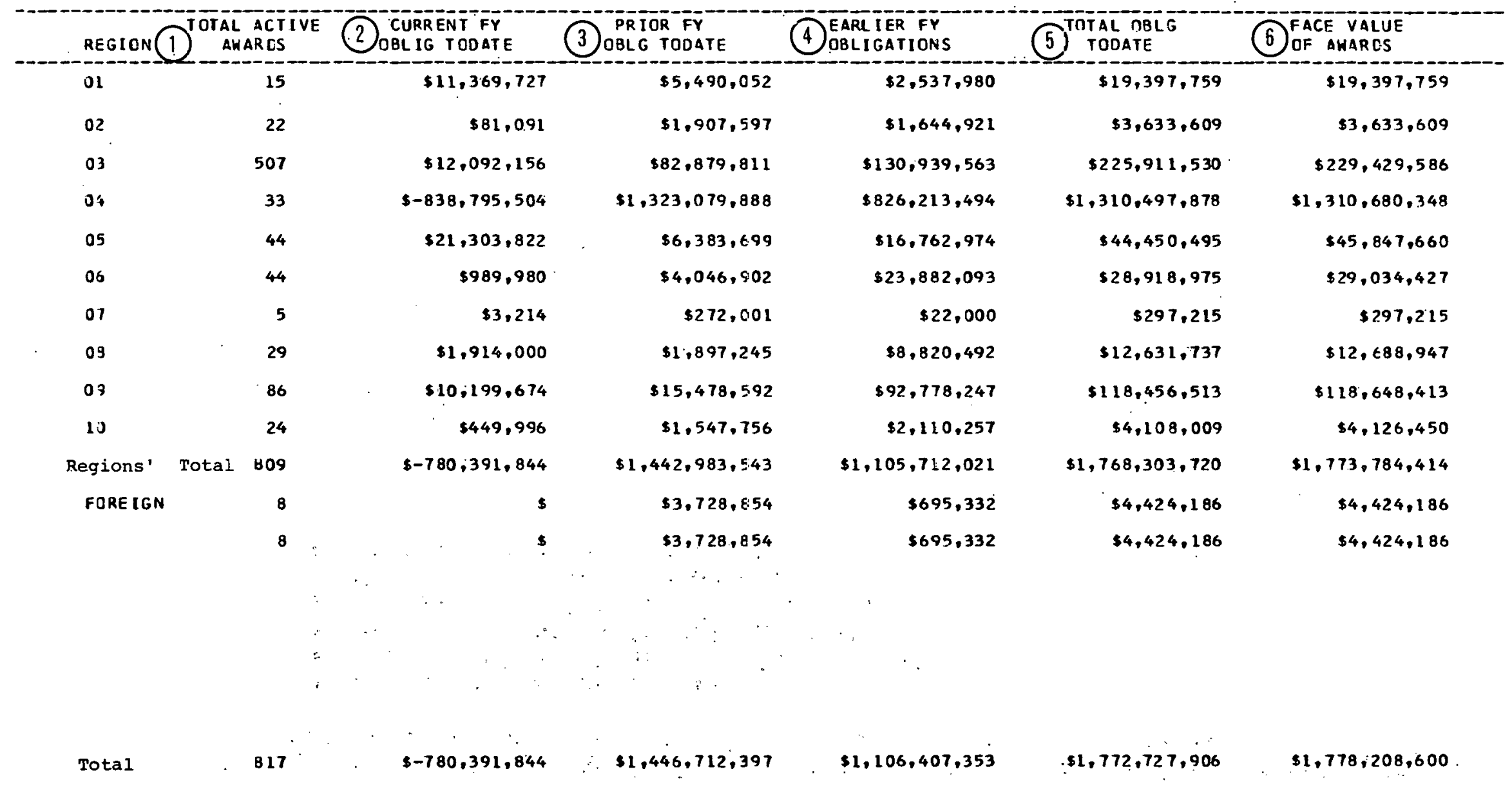




\section{UNITED STATES DEPARTMENT OF ENERGY}

SUMMARY OF PROCUREMENT AWARDS

TO NON-GCVERNMENT ENTITIES BY FEDERAL REGION--PRIME

This report includes the following data for each region and summarized for all regions, U.S. Possessions, foreign countries and all of these:

- Total Active Awards (1)

- Current Fiscal Year's Obligations (2)

- Prior Fiscal Year's Obligations (3)

- Earlier Fiscal Years' Obligations (4)

- Total Obligations to date (5)

- Face Value of Awards (6) 


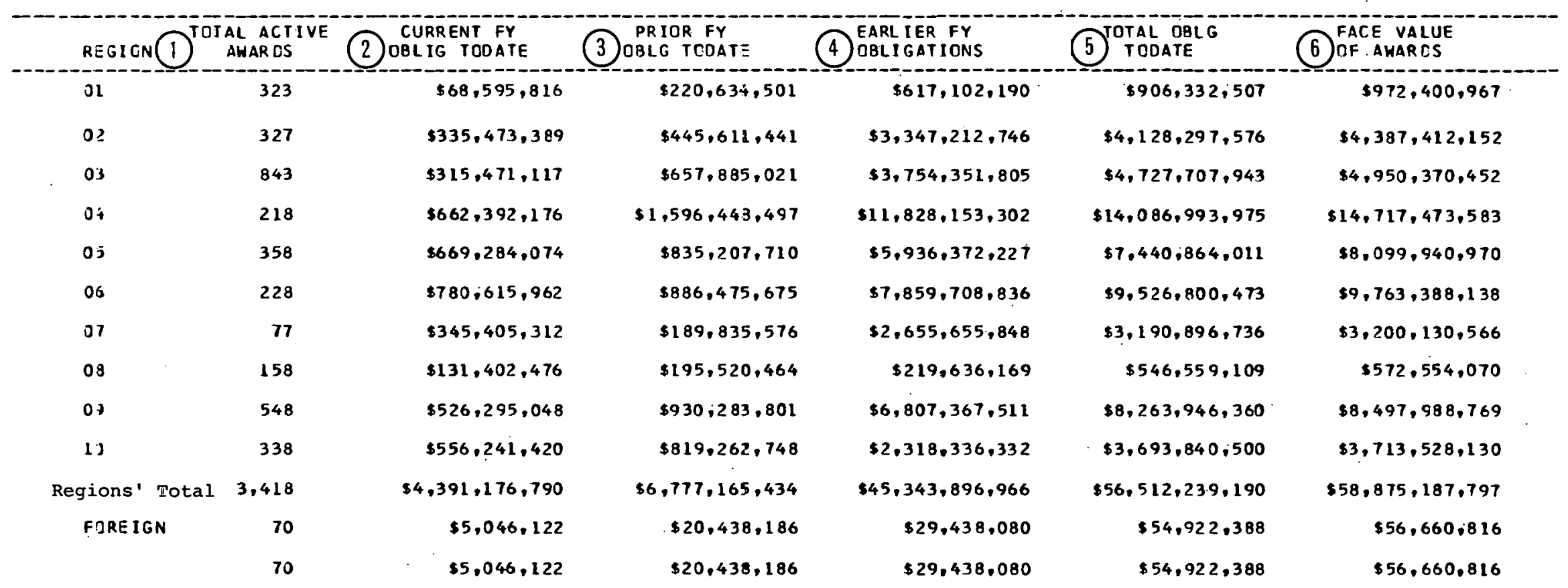


UNITED STATES DEPARTMENT OF ENERGY SUMMARY OF TOTAL FINANCIAL ASSISTANCE AWARDS

BY FEDERAL REGION

This report includes the following data for each region and summarized for all regions, U.S. Possessions, foreign countries and all of these:

- Total Active Awaras (1)

- Current Fiscal Year's Obligations (2)

- Prior Fiscal Year's Obligations (3)

- Earlier Fiscal Years' Obligations (4)

- Total Obligations to date (5)

- Face Value of Awards (6) 
IPROGRAM NUMBER - M04026511

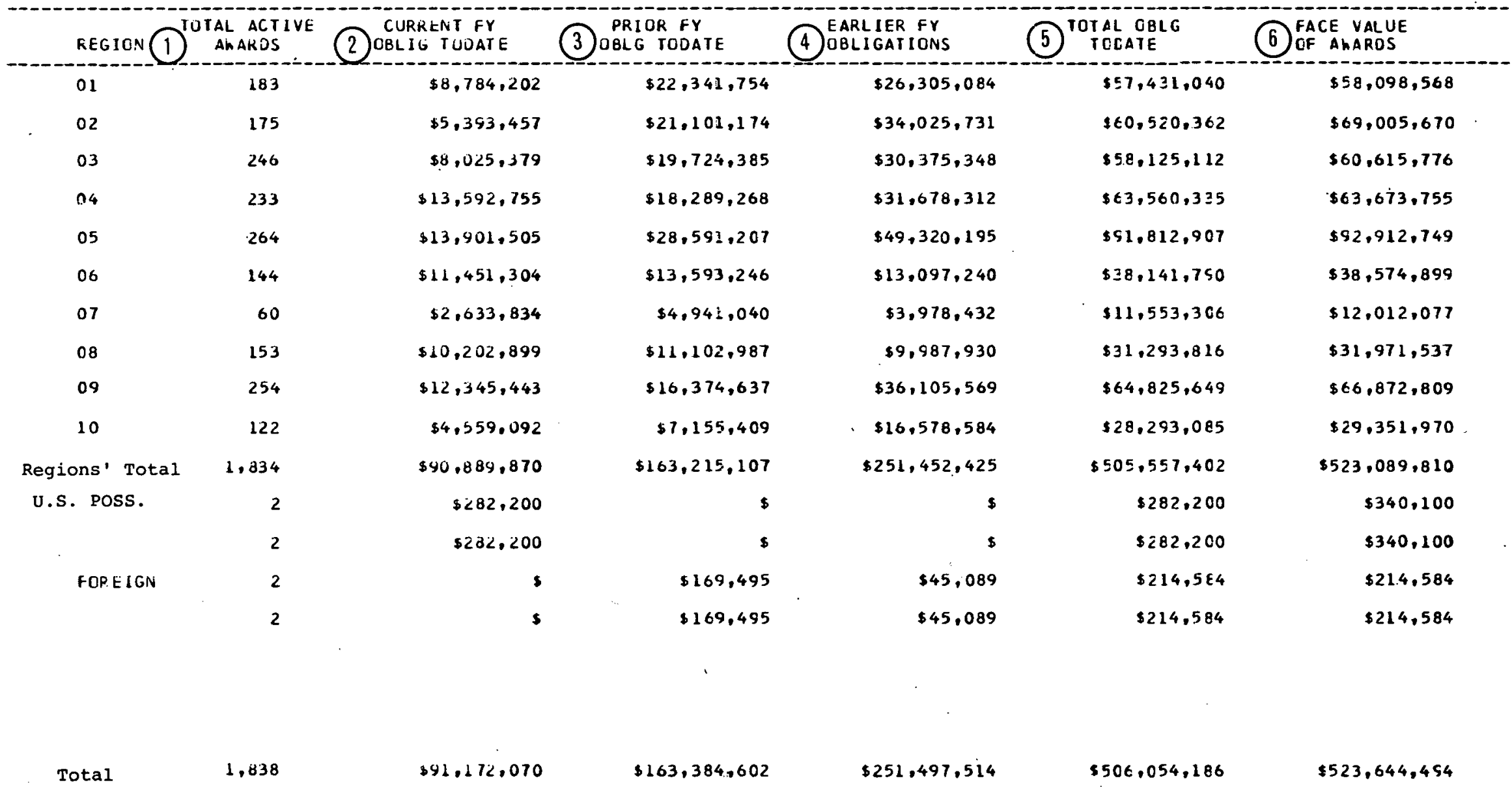


UNITED STATES DEPARTMENT OF ENERGY SUM!IARY OF FINANCIAL ASSISTANCE AWARDS TO GOVERNMENT ENTITIES BY FEDERAL REGION

This report includies the following data for each region and summarized for all regions, U.S. Possessiors, foreign countries and all of these:

- Total Active Awards (1)

- Current Fiscal lear's Obligations (2)

- Prior Fiscal Year's Obligations (3)

- Earlier Fiscal lears' Obligations (4)

- Total Obligations to date (5)

- Face Value of Awards (6) 


\begin{tabular}{|c|c|c|c|c|c|c|}
\hline REGION $1{ }^{\text {TOT }}$ & $\begin{array}{l}\text { ACT I } \\
\text { ARDS }\end{array}$ & $\begin{array}{l}\text { RRENI FY } \\
\text { IG TOOATE }\end{array}$ & $\begin{array}{l}110 R \text { FY } \\
\text { G TODATE }\end{array}$ & $\begin{array}{l}\text { LI IER FY } \\
\text { I GAT IONS }\end{array}$ & $\begin{array}{l}\text { IAL OBLG } \\
\text { TOCATE }\end{array}$ & $\begin{array}{l}\text { ACE VALUE } \\
\text { F AKAROS }\end{array}$ \\
\hline 01 & 33 & $\$ 4,106,918$ & $\$ 3,543,113$ & $\$ 2.799,623$ & $\$ 10,449.654$ & $\$ 10,449,654$ \\
\hline 02 & 23 & $\$ 93.929$ & $\$ 7.766,163$ & $62,090,153$ & $19,950,245$ & $\$ 10,126,721$ \\
\hline 03 & 35 & $\$ 2,600,861$ & $\$ 4,537,875$ & $\$ 516,031$ & $17,660,767$ & $\$ 8,989,767$ \\
\hline 04 & 58 & $\$ 8,543,100$ & $59,811,871$ & $\$ 822,099$ & $\$ 19,177,070$ & $\$ 19.177,070$ \\
\hline 05 & 38 & $\$ 6,787.126$ & $\$ 8,621,324$ & $\$ 2,008,859$ & $\$ 17,417,309$ & $\$ 17,417,309$ \\
\hline 06 & 21 & $\$ 2,750,400$ & $\$ 2,116,391$ & $\$ 649.179$ & $15,515,570$ & $\$ 5.515 .970$ \\
\hline 07 & 22 & $\$ 1,348,798$ & $\$ 3,286,747$ & $\$ 472,164$ & $\$ 5,107,709$ & $\$ 5,107,709$ \\
\hline 08 & 43 & $\$ 7,549,804$ & $\$ 3,968,863$ & $\$ 204,074$ & $\$ 11,722,741$ & $\$ 12,137,741$ \\
\hline 09 & 27 & $85,584,382$ & $\$ 1,623,066$ & $\$ 210.877$ & $\$ 7,418,325$ & $\$ 8,334,165$ \\
\hline 10 & 15 & $\$ 1,959,4<9$ & $\$ 1.142,177$ & $\$ 265,341$ & $\$ 3.36 t, 9.47$ & $\$ 3,366,947$ \\
\hline Regions' Total & 315 & $\$ 41,330,747$ & $\$ 46,417,590$ & $\$ 10,038,400$ & $\$ 57,786,737$ & $\$ 100,623,053$ \\
\hline \multirow[t]{2}{*}{ U.S. POSS. } & 2 & $\$ 282,200$ & 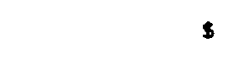 & $\$$ & $\$ 282,200$ & $\$ 340,100$ \\
\hline & 2 & $\$ 282.200$ & $\$$ & $\$$ & & $\$ 340,100$ \\
\hline Total & 317 & $\$ 41,612,947$ & $\$ 46,417,590$ & $\$ 10.038,400$ & $558,068,937$ & $\$ 100,963,153$ \\
\hline
\end{tabular}


UNITED STATES DEPARTMENT OF ENERGY

SUMMARY OF FINANCIAL ASSISTANCE AWARDS

TO NON-GOVERNMENT ENTITIES BY FEDERAL REGION

This report includes the following data for each region and summarized for all regions, U.S. Possessions, foreign countries and all of these:

- Total Active Awards (1)

- Current Fiscal Year's Obligations (2)

- Prior Fiscal Year's Obligations (3)

- Earlier Fiscal Years' Obligations (4)

- Total Obligations to date (5)

- Face Value oミAwards (6) 
BY FEUERAL REGION - NON GJVERNMENT

(PROGRAM NUMBER - M04026511

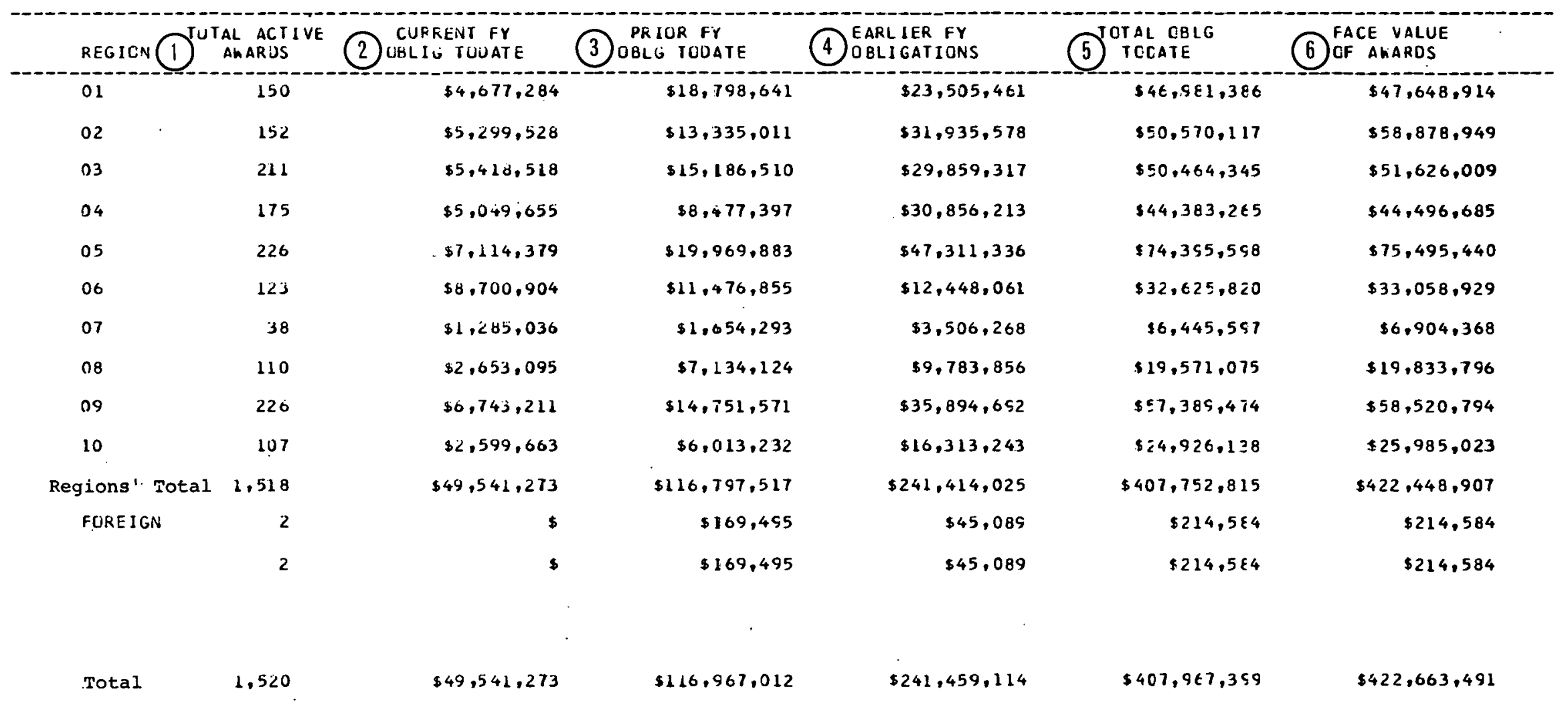


UNITED STATES DEPARTMENT OF ENERGY

SUMMARY OF TOTAL DE?ARTMENT PROCUREMENT AND FINANCIAL ASSISTANCE

OBLIGATIONS BY STATE

This report includes the following data for each state and summarized for all States, U.S. Possessions, foreign countries and all of these:

- Total Active Awards (1)

- Current Fiscal Year's Obligations (2)

- Prior Fiscal Year's Obligations (3)

- Earlier Fiscal Years' Obligations (4)

- Total Obligations to date (5)

- Face Value of Awards (6) 
IPROGRAM NUMBER - M04026.511

\begin{tabular}{|c|c|c|c|c|c|c|}
\hline STATE & $\begin{array}{l}\text { AL ACTI } \\
\text { AWAR DS }\end{array}$ & $\begin{array}{l}\text { PRENT FY } \\
\text { IG TODATE }\end{array}$ & $\begin{array}{l}\text { RIOR FY } \\
\text { LG TODATE }\end{array}$ & $\begin{array}{l}\text { RLIER FY } \\
\text { LIGATIONS }\end{array}$ & $\begin{array}{l}\text { OTAL OBLG } \\
\text { TODATE }\end{array}$ & $\begin{array}{l}\text { ACE VALUE } \\
\text { F AWARDS }\end{array}$ \\
\hline MISCONSIN & 37 & $\$ 1,452,373$ & $\$ 4,002,991$ & $\$ 8.274 .760$ & $\$ 13,730,124$ & $\$ 13,734,455$ \\
\hline WYCMING & 14 & $\$ 868,700$ & $\$ 679.992$ & $\$ 807,501$ & $\$ 2.356 .193$ & $\$ 2,356,193$ \\
\hline States' Total & 1,834 & $\$ 90,889,870$ & $\$ 163,215,107$ & $\$ 251.452,425$ & $\$ 505,557,402$ & $\$ 523,089,810$ \\
\hline \multirow[t]{2}{*}{ GJA,Y } & 2 & $\$ 282,200$ & $\$$ & s & $\$ 282,200$ & $\$ 340,100$ \\
\hline & 2 & $\$ 282,200$ & $\$$ & $\$$ & $\$ 282,200$ & $\$ 340,100$ \\
\hline AUSIRIA & 1 & $\$$ & $\$ 110,000$ & $\$$ & $\$ 110,000$ & $\$ 110,000$ \\
\hline \multirow[t]{2}{*}{ CANADA } & 1 & $\$$ & $\$ 59,495$ & $\$ 45,089$ & $\$ 104,584$ & $\$ 104,584$ \\
\hline & 2 & $\$$ & 1169,495 & $\$ 45,089$ & $\$ 214,584$ & $\$ 214,584$ \\
\hline Total. & 1,838 & $.891,172,070$ & $\$ 163,384,602$ & $\$ 251.497,514$ & $\$ 506,054,186$ & $\$ 523,644,4.94$ \\
\hline
\end{tabular}


UNITED STATES DEPARTMENT OF ENERGY

SUMMARY OF DEPARTMENT PROCUREMENT AND FINANCIAL ASSISTANCE OBLIGATIONS TO GOVERNMENT ENTITIES BY STATE

This report includes the follcwing data for each state and summarized for all States, U.S. Possessions, foreign countries and ali of these:

- Total Active Awards (1)

- Current Fiscal Year's Dbligations (2)

- Prior Fiscal Year's Obligations (3)

- Earlier Fiscal Years' Obligations (4)

- Total Obligations to date (5)

- Face Value of Awards (6) 
IPROGRAM NUMBER - MO4026511

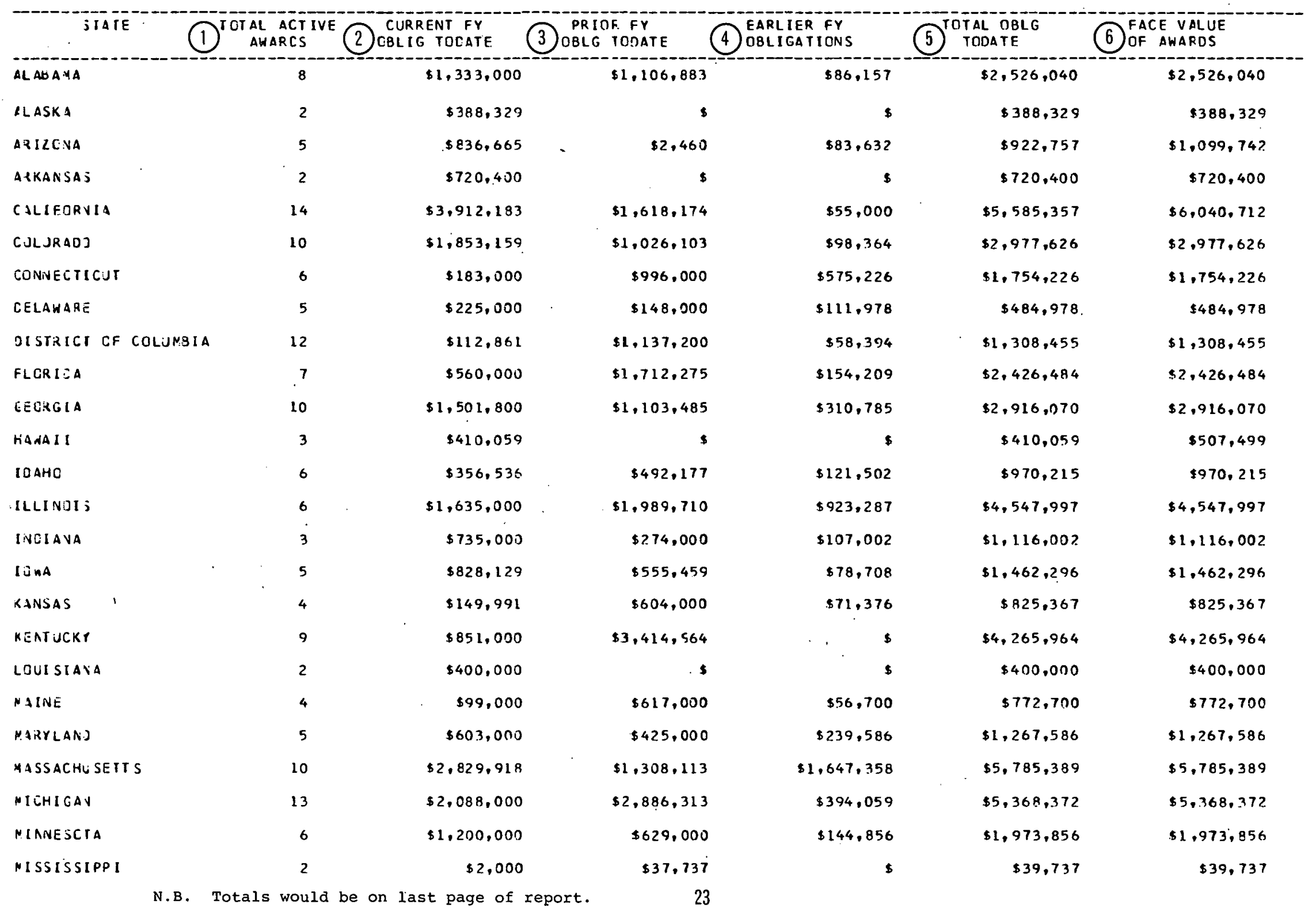




\begin{abstract}
UNIZED STATES DEPARTMENT OF ENERGY
SUMMARY OF JEPARTMENT PROCUREMENT AND FINANCIAL ASSISTANCE

OBLIGATIONS TO NON-GOVERNMENT ENTIRIES BY STATE
\end{abstract}

This report inclides the following data for each state and summarized for all States, U.S. Possessions, foreign countries and all of these:

- Total Active Awards (1)

- Current Fiscal Year's Obligations (z)

- Prior Fiscal Year's Obligations (3)

- Earlier Fiscal Years' Obligations ( 4 )

- Total Obligazions to date (5)

- Face Value of Awards (6) 
(PROGRAM NUMBER - M0402651)

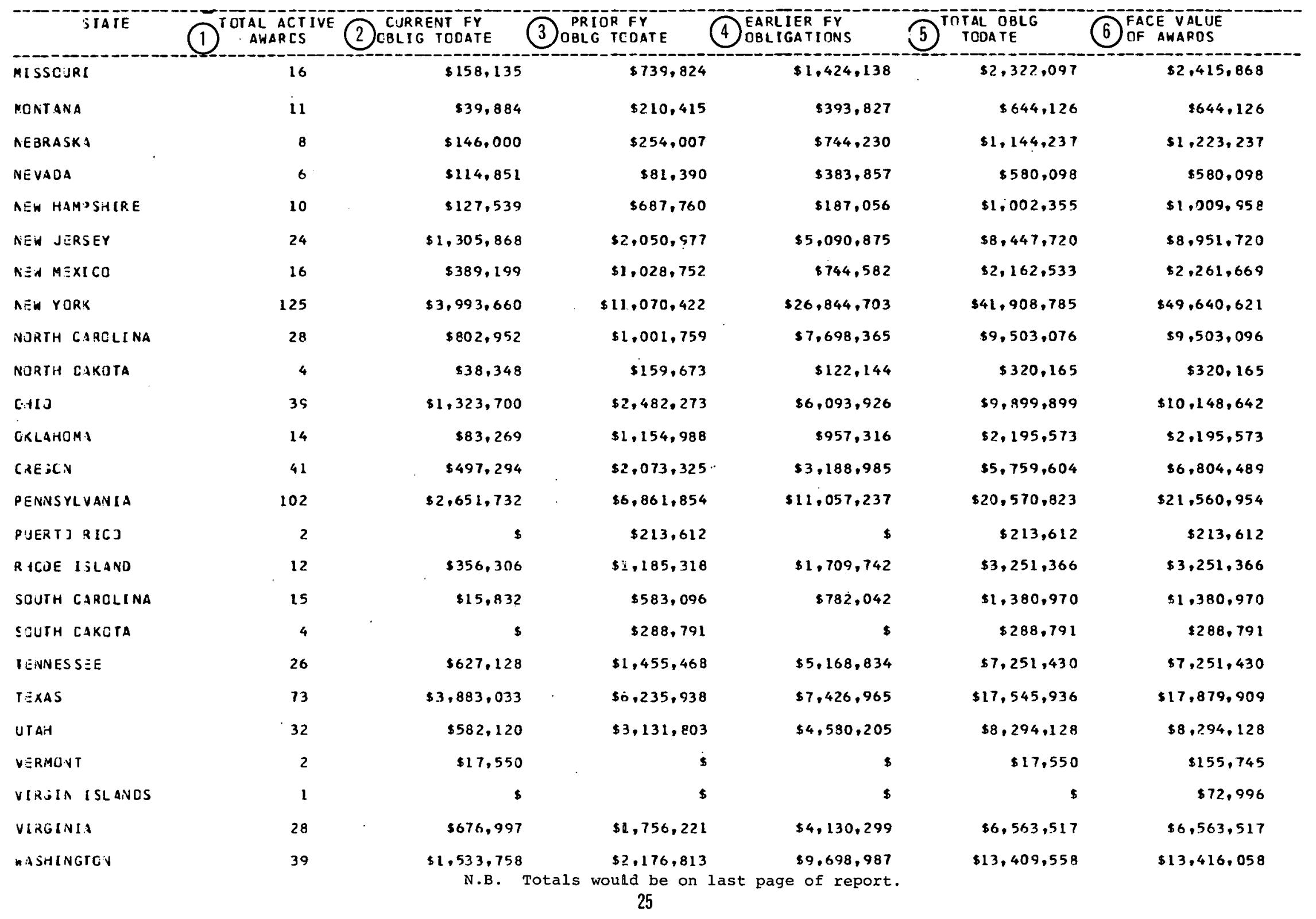


UNITED STATES DEPARTMENT OF ENERGY

SUMMARY OF TOTAL PROCUREMENT AWARDS

BY STATE--PRIME

This report inclides the following data for eash state and summarized for all States, U.S. Possessions, foreign countries and all of these:

- Total Active Awards (1)

- Current Fiscal Year's Obligations (2)

- Prior Fiscal Year's Obligations (3)

- Earlier Fiscal Years' Obligations (4)

- Total Obligations to date (5)

- Face Value of Awards (6) 
BY STATE-PRIME

(PROGRAM NUMBER - MO402651)

\begin{tabular}{|c|c|c|c|c|c|c|c|}
\hline STATE & $1^{r}$ & $\begin{array}{l}\text { AL ACT IVE } \\
\text { AWARDS }\end{array}$ & $\begin{array}{l}\text { CURRENT FY } \\
\text { OBLIG TOOATE }\end{array}$ & $\begin{array}{l}\text { PRIOR } \\
\text { OBLG TODATE }\end{array}$ & $\begin{array}{l}\text { EARLIER FY } \\
\text { OBLIGATIONS }\end{array}$ & (5) TOOATE & $\begin{array}{l}\text { D) FAC AHARDS } \\
\text { OF }\end{array}$ \\
\hline WISCCNSIN & & 47 & $\$ 2,520,753$ & $\$ 6 ; 799,753$ & $\$ 44,956,075$ & $\$ 54,276,581$ & $\$ 54,480,912$ \\
\hline WYCH ING & & 21 & $\$ 1,095,487$ & $\$ 20.962,424$ & $\$ 11,875,331$ & $\$ 33,933,242$ & $\$ 34,158,549$ \\
\hline States' & Total & 6,066 & $\$ 3,701,674,816$ & $\$ 8,383,408,084$ & $\$ 46,701,293,912$ & $\$ 58,786,376,812$ & $\$ 61,172,338,521$ \\
\hline \multirow[t]{2}{*}{ G JAM } & & 2 & $\$ 282,200$ & $\$$ & $\$$ & $\$ 282,200$ & $\$ 340,100$ \\
\hline & & 2 & $\$ 282,200$ & s & s & $\$ 282,200$ & $\$ 340,100$ \\
\hline AUSTRIA & & 2. & s & $\$ 171,400$ & $\$ 68,500$ & $\$ 239,900$ & $\$ 239,900$ \\
\hline RELGIJMM & & 3 & $\$$ & $\$ 60,000$ & $\$ 65,000$ & $\$ 125,000$ & $\$ 125,000$ \\
\hline CANADA & & 7 & $\$ 2,178$ & $\$ 280,692$ & $\$ 142,808$ & $\$ 425,678$ & $\$ 425,678$ \\
\hline CENMARK & & 1 & $\$$ & $\$ 146,000$ & $\$$ & $\$ 146,000$ & $\$ 146,000$ \\
\hline ENGLAND & & 8 & s & $53,656,776$ & $\$ 1,882,830$ & $55,539,606$ & $\$ 5,539,606$ \\
\hline FINLAND & & 1 & $\$ 75,275$ & $\$ 75,275$ & $\$ 455,807$ & $\$ 606,357$ & $\$ 606.357$ \\
\hline FRANCE & & 7 & $\$ 1,032,800$ & $\$ 785.511$ & $, \$ 3,224,670$ & $\$ 5,042,981$ & $\$ 5,072,981$ \\
\hline IGELANC & & 2 & s & $\$ 84,850$ & $\$ 919.766$ & $\$ 1.004 .616$ & $\$ 1,004,616$ \\
\hline I SRAEL & & 2 & $\$$ & $\$ 11,900$ & $\$ 406,659$ & $\$ 418,559$ & $\$ 418,559$ \\
\hline IT ALY & & 4 & $\$ 352$ & $\$ 254,550$ & $\$ 874.248$ & $\$ 1,129,150$ & $\$ 1,594,010$ \\
\hline JAPAN & & 19 & $\$ 3,879,728$ & $\$ 18,362,494$ & $\$ 17,095,901$ & $\$ 39,338,123$ & $\$ 40,438,245$ \\
\hline SOUTH AFRICA & & 1 & $\$$ & $\$$ & s & 5 & $\$$ \\
\hline S AEDEN & & 7 & $\$ 44.764$ & $\$ 126,844$ & $\$ 2,409,038$ & $\$ 2,580,646$ & $\$ 2,703,892$ \\
\hline S IITZERL AND & & 9 & $\$ 11,025$ & $\$ 148,803$ & $\$ 2.427,643$ & $\$ 2,587,471$ & $\$ 2.607 .671$ \\
\hline UNITED KINGDOM & & 1 & $\$$ & $, \$ 151,440$ & $\$$ & $\$ 151,440^{\circ}$ & $\$ 151,440$ \\
\hline \multirow[t]{3}{*}{ WEST GERMANY } & & 6 & $\$$ & $\$ 20.000$ & $\$ 205,631$ & $\$ 225,631$ & $\$ 225,631$ \\
\hline & & 80 & $\$ 5,046,122$ & $\$ 24,336,535$ & $\$ 30,178,501$ & $\$ 59,561,158$ & $\$ 61,299,586$ \\
\hline & TOTAL & 6,148 & $\$ 3,707,003,138$ & $\$ 8,407,744,619$ & $\$ 46,731,472,413$ & $\$ 58,846,220,170$ & $\$ 61,233,978,207$ \\
\hline
\end{tabular}


UNITED STATES DEPARTMENT OF ENERGY

SUMMARY OF PROCUREMENT AWARDS

TO GOVERNMENT ENTITIES BY STATE--PRIME

This report includes the following data for each state and summarized for all states, U.S. Possessions, foreign countries and all of these:

- Total Active Awards (1)

- Current Fiscal Year's Obligations (2)

- Prior Fiscal Year's Obligations (3)

- Earlier Fiscal Years' Obligations (4)

- Total Obligations to date (5)

- Face Value of Awards (6) 
(PROGRAM NIJMBER - M0402651)

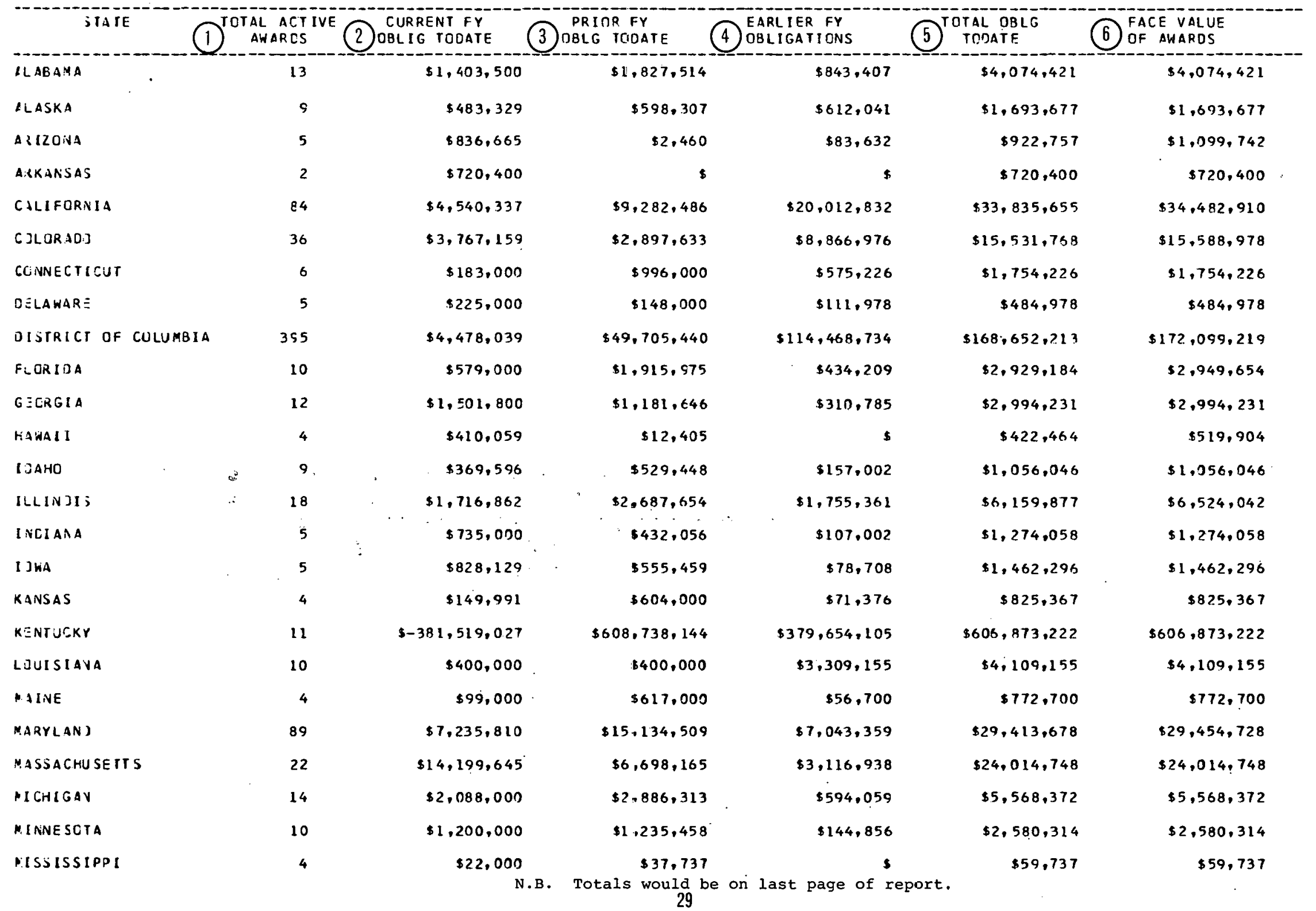


UNITED STATES DEPARTMENT OF ENERGY

SUMMARY OF PROCUREMENT AWARDS

TO NON-GOVERNMENT ENTITIES BY STATE--PRIME

This report includes the following data for each state and summarized for all States, U.S. Possessions, foreign countries and all of these:

- Total Active Airaras (1)

- Current Fiscal Year's Obligations (2)

- Prior Fiscal Year's Obligations (3)

- Earlier Fiscal Years' Obligations (4)

- Total Obligations to date (5)

- Face Value of Awards (6) 


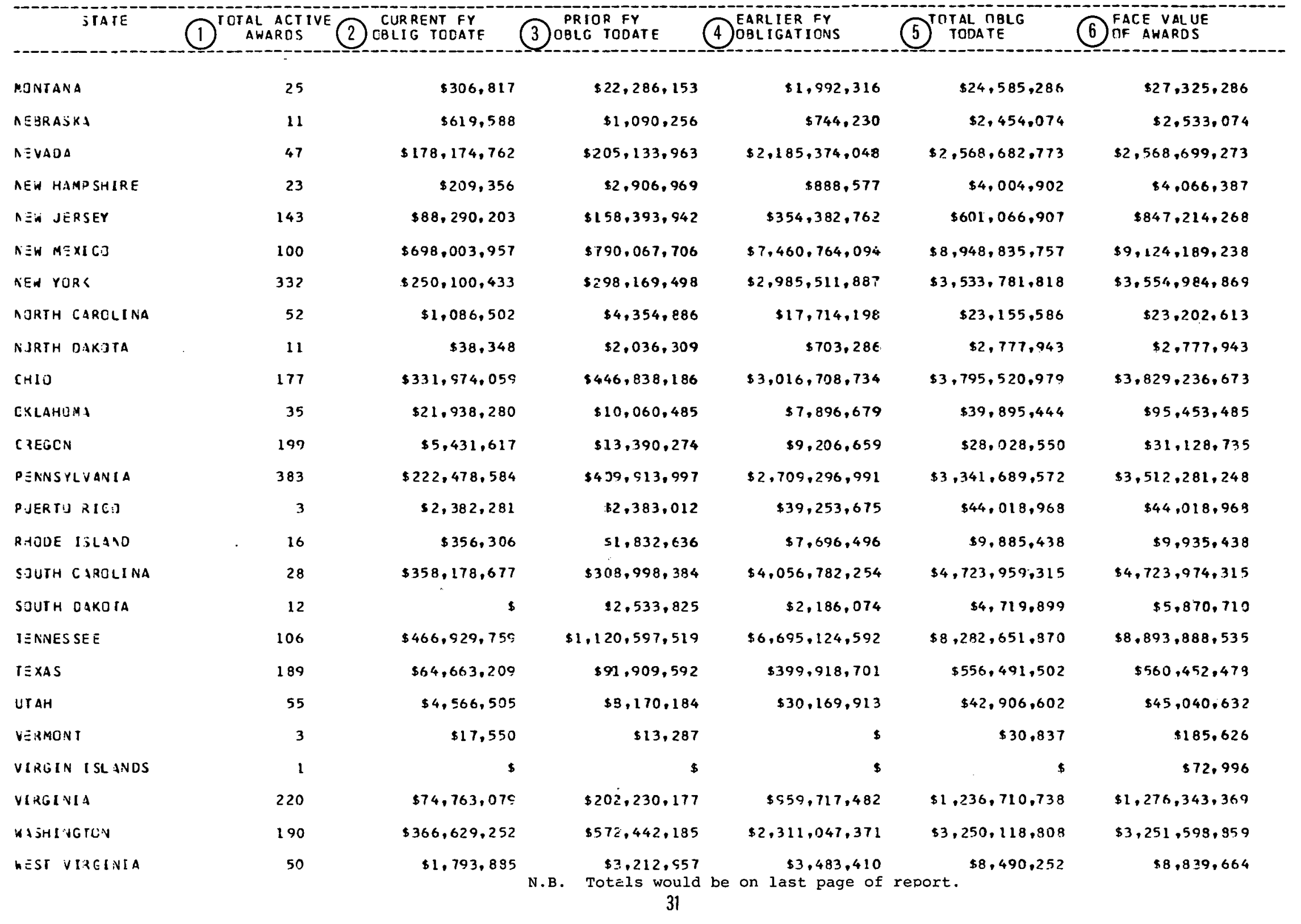




\section{UNITED STATES DEPARTMENT OF INERGY SUMNARY JF TOTAL FINANCIAL ASSISTANCE AWARDS \\ BY STATE}

This report includes the following data for each state and summarized for all States, U.S. Possessions, foreign countries and all of these:

- Total Active Awards (1)

- Current Fiscal Year's Obligations (2)

- Prior Fiscal Year's Obligations (3)

- Earlier Fiscal Years' Obligations (4).

- Total Obligations to date (5)

- Face Value of Awaras (6) 
UNI TED STATES DEPAR TMENT OF ENERGY
SUMMARY OF TOTAL FINANCI AL ASSISTANCE AHAROS

BY STATE

(PRCGRAM NUMBER - M0402651)

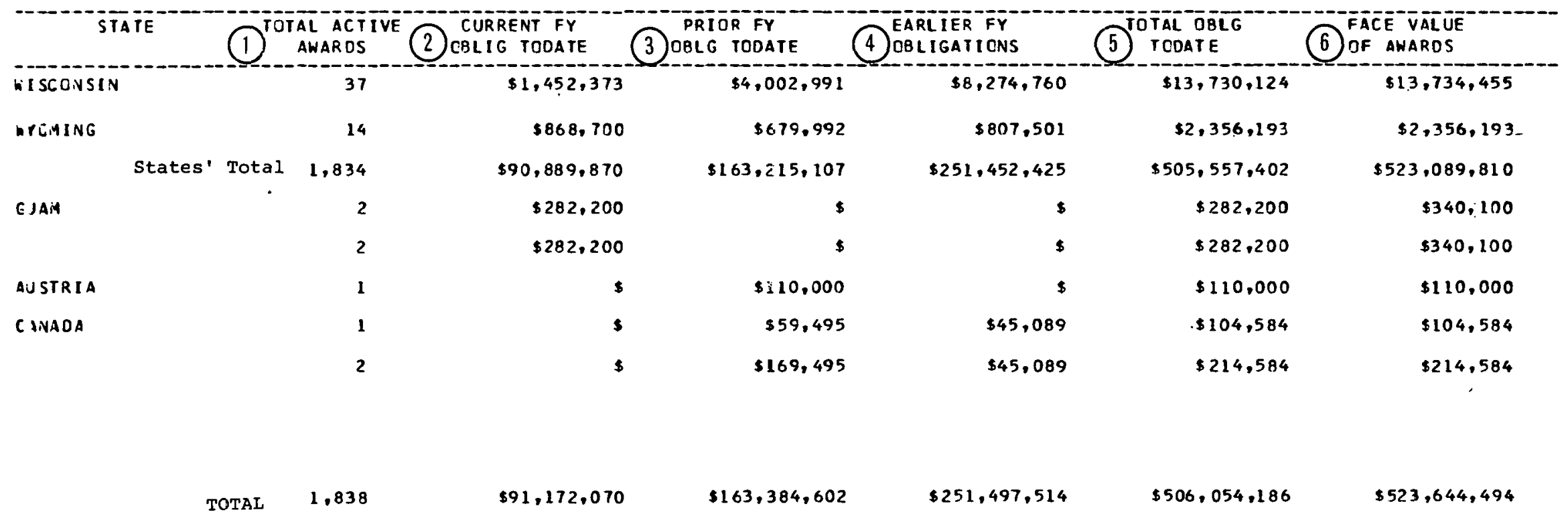


UNITED STATES DEPARTMENT OF ENERGY SUMNARY OF FINANCIAL ASSISTANCE AWARDS TO GOVERNMENT ENTITIES BY STATE

This report includes the following data for each state and summarized for all States, U.S. Possessions, foreign countries and all of these:

- Total Active Awards (1)

- Current Fiscal Year's Obligations (2)

- Prior Fiscaí Year's Obligations (3).

- Earlier Fiscal Years' Obligations (4)

- Total Obligations to date (5)

- Face Value of Awards (6) 
CPROGRAM BY STATE

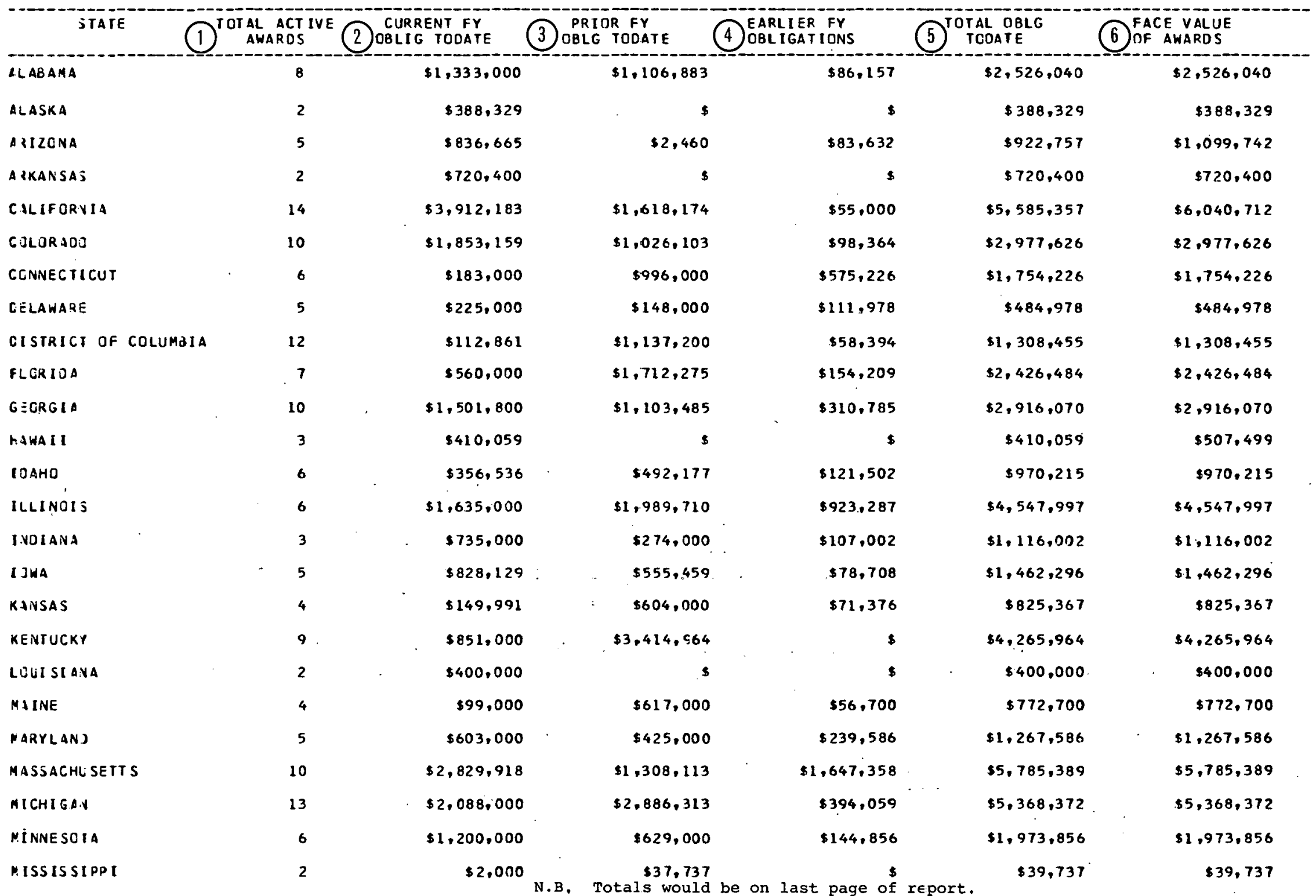


UNITED STATES DEPARTMENT OF ENERGY SUMMARY CF FINANCIAL ASSISTANCE AWARDS TO NON-GOVERNMENT ENTITIES BY STATE

This report includes the following data for each state and summarized for all States, U.S. Possessions, foreign countries and all of these:

- Total Active Awards (1)

- Current Fiscal Year's Obligations (2)

- Prior Fisical Year's Obligations (3)

- Earlier Fiscai Years' Obligations (4)

- Total Obligations to date (5)

- Face Value of Awards (6) 


$$
\text { BY STATE }
$$

(PROGRAM NUMBER $=$ M0402651)

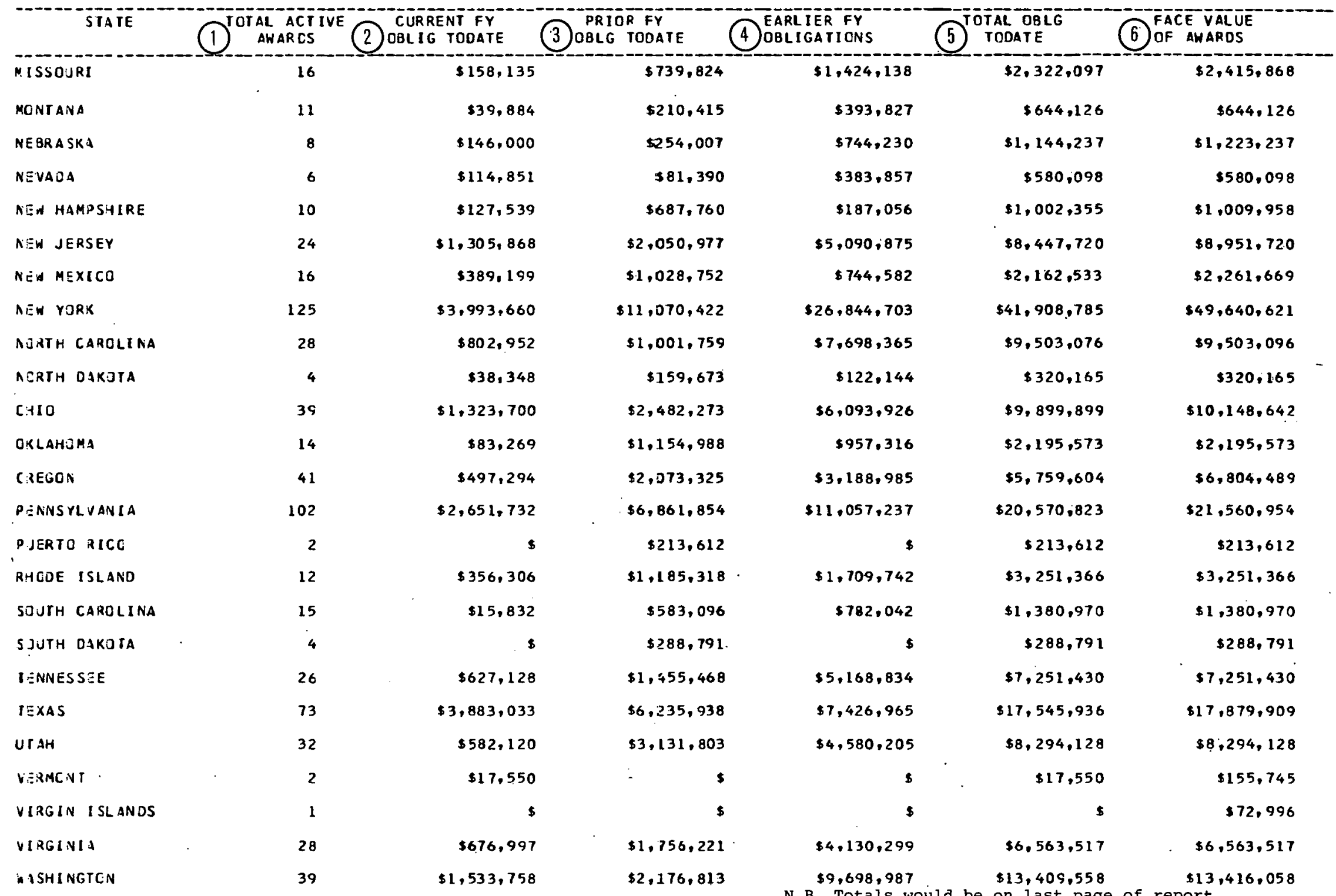


III. Unpublished Reports 
THIS PAGE

\section{WAS INTENTIONALLY LEFT BLANK}




\section{ACTIVE PRIME CONTRACTS BY STATE, BY PROGRAM}

This monthly report lists active prime contracts by program under each state. It provides:

- Organizational Code (2)

- Contract Number (3)

- Task Order or Project Agreement Number (4)

- Award Date (5)

- Total Cumulative Obligations (6)

- City and State Work Site (7)

- Contract Status (8)

- Work Category (9)

- Budget Activity Number (10)

- Contract Type (11)

- Action Category (12)

- Completion Date (13)

- Current FY Obligation (14)

- Total Contract Amount (15)

- Congressional District Code (16)

- Procurement Source (17)

- Prior FY Obligations (18)

- Brief Work Description (19)

See Appendix for coding information.

$\approx$ 
STATE - PA

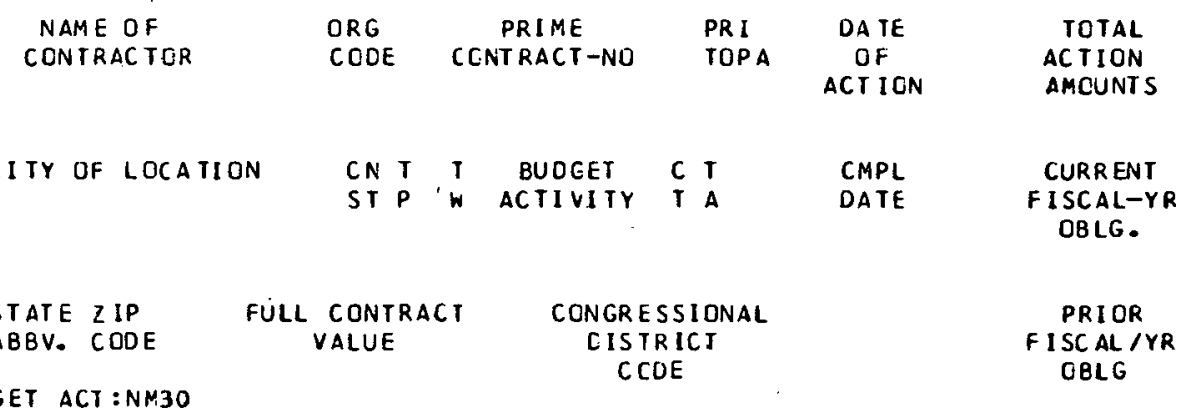

BUDGET ACT : NM3O

OBLG

BLD-ACT TOTAL

8,421

(6) $\$ 8,421$

58,421

$\$ 120,160$

WEST INGHOUSE ELECTRIC 2CHS EY-76-C-02-4003 506/15/76 MADISON

$\begin{array}{lllll}10 & 8 & 3\end{array}$ $07 / 31 / 77$

ELC-ACT TUTAL

$\$ 120,160^{\circ}$

EUDGET ACT:RTOL

(1) Alleghenit general hosp PITTSBURGH

$$
\text { PA } 15212
$$

PITISBURGH UNIV OF (7) PITTSBURGH

$$
\text { PA } 15261
$$

\section{(3)}

CH9 EY-76-S-02-3097 $06 / 01 / 70$ $108 \quad 3(10$ RT 01 10821495

CH9 EE-77-S-02-4552
8. $108.3 \quad$ RTO1 SO

(17) $09 / 01 / 77$ $05 / 31 / 78$

$04 / 19 / 71$

WESTINGHOUSE ELECTRIC LARGE PA 15025

BLDACT TOTAL

BUOGET ACT :R 102

CREXEL UNIVERS ITY PENNSYLVANIA

$$
\text { PA } 1910.4
$$

CH9 EY-76-C-02-3043 (15) $9.940^{3} .372^{\text {RTO1 }} 42 \quad 20^{P}$ D

$12 / 31 / 77$

\section{$9,954,899$}

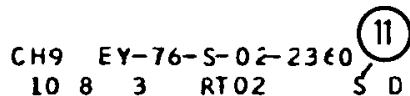

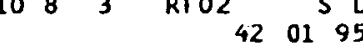

$08 / 01 / 75$

$10 / 31 / 76$

pennsylvania state univ cha ey-76-5-02-2223 4 $\mathrm{xX} 05101172$ UNI VERSI TY PK PA 16802
1083 RTO2 32395 (13) $10 / 31 / 77$

(9)

\begin{abstract}
$42 \quad 2395$
\end{abstract}
$4 !$
DESCR IPTION

CF WORK
$\$ 200,234$

(18) $537,290^{\circ}$

14,527

14,527

$10,651,719$

$1,648,531$ $10,866,480$ (14) $1,700,348$

$\$ 326,982$ 19 EFFECTS OF MES OSCALE WEATHER DISTURBANCES ON $\$ 64,200$

151,929

28,890 SYS FORMERLY-AT-30-1-4205 AND FRCM hOFKEFS AT FERC CE FCRMERLY-AT-30-1-4277

CONTAMINATION CONCENTRATIONS

SPIN-LABELED MEMBRANES

MODEL SYSTEMS WILL ATTEMPT TO CHARACTERIZE
STUOY TO EVALUATE

ION HYBRID REACTOR PLANT

FEASIB IL ITY OF LASER FUS

EFFECT OF RADIATICN CN NORMAL HEMATOPOIESIS AN D ON VIRAL INDUCED CANCERS OF THE HEMATOPOIETIC

AN EVALUATION OF CARBON MONOXIOE IN BLGOC SAMPLE. $S$ FROM THE SECOND HEALTH AND NUTRITION SURVEY

EVALUATION OF PRACTI CABILITY OF A RADIOISGTOPE THERMAL CONVERTER FOR AN ARTIFIC IAL HEART DEVI

ROTATIONAL AND TRANSLATIONAL MOLECULAR MOTIO 
ACTIVE PRIME CONTRACTS BY PROGRAM, BY STATE

This monthly report lists active prime contracts by state under each program. It provides:

- Contractor (1)

- Organizational Code (2)

- Contract Number (3)

- Task Order or Project Agreenent Number (4)

- Award Date (5)

- Total Cumulative Cbligations (6)

- City and State Work Site (7)

- Contract Status (8)

- Work Category (9)

- Budget Activity Number (10)

- Contract Type (11)

- Action Category (12)

- Completion Date (13)

- Current FY Obligation (14)

- Total Contract Amount (15)

- Congressional District Code (16)

- Procurement. Source (17)

- Prior FY Obligations (18)

- Brief Work Description (19)

See Appendix for coding infcrmation. 
FRCGFAM: $39 K K$

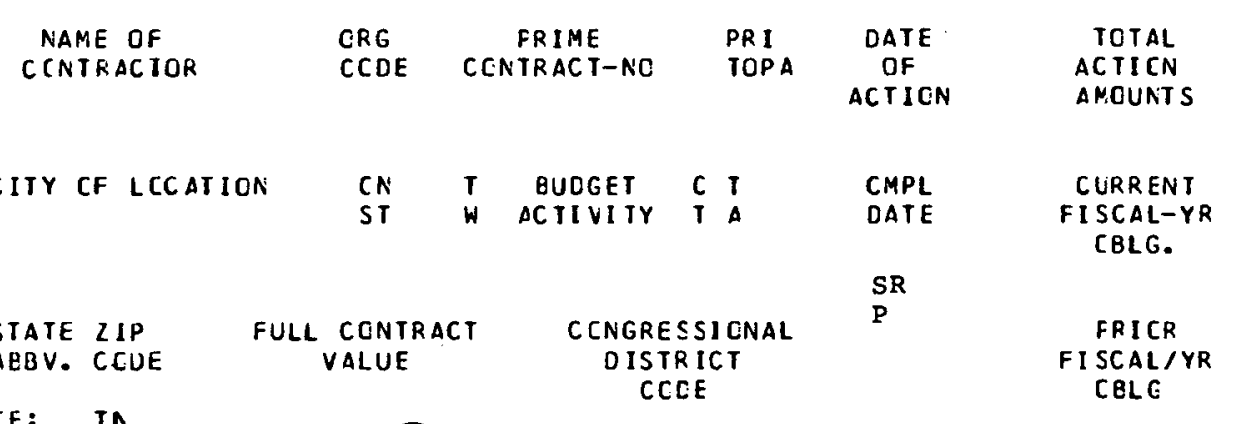

STATE: TA

(1)Tiptca e reynclos

CAK RICGE TN 37830
(5) $04 / 20 / 77$ $05 / 05 / 78$ 10525 39KK
$611,586 \quad 470395$

OR9 EY-77-C-05-5350 1052 3SKK F D 1307/29/79 $2,369,392 \bigcirc 40395$

TITAN SOUTHERN STATES ORAK RIDGE
(7) $37830^{\circ}$

TRI STATE ROUFG LF TENA OAK RIUGE

$105{ }^{\mathrm{E}} 39 \mathrm{KK}$ F

$06 / 01 / 77$
$03 / 07 / 78$

$$
\text { TN } 37830
$$
IRI-STATE PIPING \& ENG
GAK RIDGE

$$
\text { TN } 37830
$$

S 0 R 9

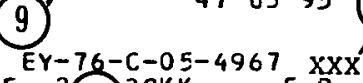

STATE TOTAL

$$
\text { STATE: UI } 6.271 .943
$$

JACGBSEN CCNSIRUCIIGN CO IC9 EY-76-C-07-1568 SALT LAKE CIIY $\quad 10622$ 39KK $10 / 10 / 77$ SALT LAKE CIIY
UT 84122

STATE TGTAL

$$
\text { (15) } 818,243^{39 k K} 1 \epsilon 02^{2} 95 \text { (12) }
$$

$12 / 12 / 75$
$01 / 04 / 77$ (17) $\mathrm{xxx}$

\section{(6)} $\$ 611,58$ CONSTRUCTION GF GUARD

DESCR I PTION OF WCRK

CASHLIAE TCTAL

$$
818,243
$$

\section{2,369,392 19 CONSTRUCT INTERMEDIATE LEVEL WASTE NANAGEMENT} $2,369,392$

$2,5 \varepsilon 7, \varepsilon 67$

$2,587, \varepsilon \in 7$

$1,047,583$

4.633

$\$ 22,379,121$

$\$ 16,586,588$

$\$ 818,243$ $\$ 9,202$ $\$ 31,034$

CONSTRUCTION CF CPP FACILITY

RERDOFING OF BUILDINGS K-301-1 THRU K-312-3 CAK RIDGE SASEOUS DIFFUSICN PLANT CONSTRIJCTION OF K-892-C CLARIFIER OAK RIDGE EASE
OUS DIFFUSION PLANT

\section{$14 \stackrel{818,243}{\$, 202}$} $14) \$ 39,202$

$\$ 55,055,712$

(18) $\$ 25,942,815$

$t E, 249,35 t$ 
This monthly report lists alphabetically all active contracts. It provides:

- Contractor (1)

- Organizational Code (2)

- Contract Number (3)

- Task Order or Project Agreement Number. (4)

- Award Date (5)

- Total Cumulative Obligations (6)

- City and State Nork Site (7)

- Contract Status (8)

- Work Category (9)

- Budget Activity Number $\left(z_{-}\right)$

- Contract Type (11)

- Action Category (12)

- Completion Date (13)

- Current FY Obligation (14)

- Total Contract Amount (15)

- Congressional District Code (16)

- Procurement Source (17:

- Prior FY Obligations (18)

- Brief Work Description (19)

See Appendix for coding information. 


\begin{tabular}{|c|c|c|c|c|c|c|}
\hline $\begin{array}{l}\text { NAME UF } \\
\text { CCINTRALIUR }\end{array}$ & $\begin{array}{l}\text { UKKG } \\
\text { CULE }\end{array}$ & $\begin{array}{l}\text { PRIME } \\
\text { CONTRACT-NO }\end{array}$ & $\begin{array}{l}\text { PF.I } \\
\text { TGPA }\end{array}$ & $\begin{array}{l}\text { UATE } \\
\text { UF } \\
\text { ACTIUN }\end{array}$ & $\begin{array}{l}\text { TOIAL } \\
\text { ACTION } \\
\text { AMOUNTS }\end{array}$ & $\begin{array}{l}\text { DESCRIPIICK } \\
\text { OF hORK }\end{array}$ \\
\hline CITY OF LOCATIUN & $\begin{array}{l}\text { CN } \\
\text { S I }\end{array}$ & $\begin{array}{l}\text { T BUULET } \\
\text { W C } \\
\text { ACTIVITY I }\end{array}$ & $\begin{array}{ll}\text { I } & S K \\
\text { A } & P\end{array}$ & $\begin{array}{l}\text { CMPL } \\
\text { OATE }\end{array}$ & $\begin{array}{l}\text { CURRENT } \\
\text { FISCAL-YR } \\
\text { CBLG. }\end{array}$ & \\
\hline $\begin{array}{l}\text { SIATE ZIP } \\
\text { ABBV. CUJE }\end{array}$ & $\begin{array}{l}\text { CUNTK } \\
\text { VALUE }\end{array}$ & $\begin{array}{l}\text { CUNUKESS } \\
\text { OISTRL } \\
\text { CUUE }\end{array}$ & & & $\begin{array}{l}\text { PRIOR } \\
\text { FISCALIYR } \\
\text { OBLG }\end{array}$ & \\
\hline $\begin{array}{c}\text { WILL IAM TAU \& ASSUC INC } \\
\text { SI LCLIS } \\
\text { MO } E 3110\end{array}$ & $\begin{array}{r}\text { WA2 } \\
10\end{array}$ & 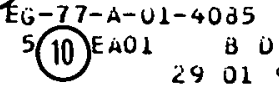 & 020 & $\begin{array}{l}08 / 31 / 77 \\
08 / 30 / 82\end{array}$ & $\begin{array}{r}\$ 9,512 \\
\$ 9,512\end{array}$ & SOLAR SPACE HEATING AND HOI WATER SYSTEM \\
\hline $\begin{array}{l}\text { WILLIAMS OG G SKAGOS KL } \\
(7)^{\text {LAS VEGAS }} 8910 \mathrm{~s}\end{array}$ & $\begin{array}{r}\text { ALQ } \\
10\end{array}$ & 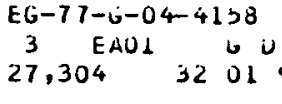 & $\underset{95}{20}$ & $\begin{array}{l}09 / 30 / 77 \\
04 / 30 / 78\end{array}$ & $\begin{array}{l}27,304 \\
27,304\end{array}$ & SOLAR PAFAEOLIC IHROUCH FORMING PRCCESS \\
\hline $\begin{array}{l}\text { WILLIAMS PATENT.CHUSHER } \\
\text { ST. LCUIS } \\
\text { MO } 63102\end{array}$ & $8)_{10}^{\mathrm{CH} 9}$ & $\begin{array}{l}E Y-77-C-02-4200 \\
5 \quad 16 \quad 29 \text { OADI }\end{array}$ & $\underset{\$ 5}{20}$ & $\begin{array}{l}01 / 19 / 77 \\
08 / 31 / 77\end{array}$ & $\begin{array}{l}88,096 \\
88,096\end{array}$ & $\begin{array}{l}\text { SUPPLY CCVTRACT FGR CEALPULVERIZING AND DRYING } S \\
\text { YSTEM }\end{array}$ \\
\hline $\begin{array}{l}\text { WILLIAMS/TREUILCUCK/WHIT } \\
\text { PENNSYLVANIA } \\
\text { FA } 13 L 12\end{array}$ & $\begin{array}{r}8 M 1 \\
10\end{array}$ & 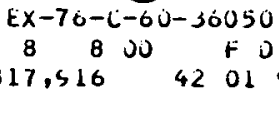 & $\begin{array}{l}0 \\
05 \\
020\end{array}$ & $\begin{array}{l}06 / 15 / 77 \\
06 / 16 / 77\end{array}$ & $\begin{array}{l}317,916 \\
317,916\end{array}$ & $\begin{array}{l}\text { UESIGA CF COAL PFEPARATICA FRCCESS CEVELOPMENT F } \\
\text { ACILITY }\end{array}$ \\
\hline $\begin{array}{l}\text { WILMOT-FLEMINL ENGK. CO } \\
\text { PHILACELPHIA } \\
\text { PA } 19111\end{array}$ & $\begin{array}{r}\text { CH9 } \\
10\end{array}$ & $\begin{array}{l}E Y-77-\lambda-02-4071 \\
5.35 \mathrm{NN} \quad F P\end{array}$ & 20 & $\begin{array}{l}11 / 26 / 76 \\
09 / 30 / 77\end{array}$ & $\begin{array}{l}16,005 \\
16,005\end{array}$ & FABRICATI:N CF SARPLE \\
\hline $\begin{array}{l}\text { WILSEY } \& \text { HAM } \\
\text { FUSTER CITY } \\
\text { CA } 94404\end{array}$ & $\begin{array}{r}S F \theta \\
10\end{array}$ & 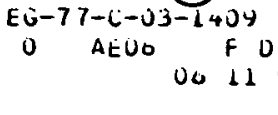 & 20 & $\begin{array}{l}03 / 01 / 77 \\
02 / 28 / 78\end{array}$ & & ANALYZE GECTHEFMAL LOAN GLARANTY APPLICATICNS \\
\hline $\begin{array}{l}\text { WILSUN GERALU L } \\
\text { WAYLAND } \\
\text { MA } 01778\end{array}$ & ${ }^{n A 2}$ & $\left\{\begin{array}{l}\mathrm{C}-77-\lambda-01-3001 \\
\mathrm{HAOL}\end{array}\right.$ & 20 & $\begin{array}{l}11 / 05 / 76 \\
01 / 31 / 78\end{array}$ & $\begin{array}{l}7,391 \\
7,391\end{array}$ & $\begin{array}{l}\text { REPORTS CENCEFNING THE FRELIMINARY DESIGNS OF L } \\
\text { ARGE SUPERCONCLCTING CENERATURS }\end{array}$ \\
\hline $\begin{array}{l}\text { WIRE INSTALLATION CONT } \\
\text { HCT SPRINGS } \\
\text { MT } 5 S 845\end{array}$ & $\begin{array}{r}8+1 \\
10\end{array}$ & 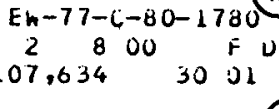 & $\overbrace{\substack{y \\
u_{0}}}$ & $\begin{array}{l}09 / 07 / 77 \\
11 / 04 / 77\end{array}$ & $\begin{array}{l}107,634 \\
107,634\end{array}$ & CCNSTFLCTION \\
\hline $\begin{array}{l}\text { WIRTH ASSOCIATES } \\
\text { BILLINGS } \\
\text { MT } 59102\end{array}$ & $\frac{10}{15}$ & $\begin{array}{l}E W-77-L-80-1930 \\
008 \text { v0 } \\
10,000 \quad 3002\end{array}$ & $\underbrace{(4)}_{30} \times x$ & $\begin{array}{l}09 / 06 / 77 \\
10 / 15 / 77\end{array}$ & 14 $\int_{10,000}^{10,000}$ & SERVICES \\
\hline $\begin{array}{l}\text { WISCONSIN STATE UF } \\
\text { MADISON } \\
\text { WI } 53702\end{array}$ & $\begin{array}{r}\text { FK5 } \\
10\end{array}$ & $\begin{array}{ll}E W-76-0-45-0016 \\
8 \quad C 701 & 50 D \\
58.239 & 5502\end{array}$ & sis & $\begin{array}{l}09 / 22 / 76 \\
06 / 30 / 77\end{array}$ & 98,239 & $\begin{array}{l}\text { PROVICES GRANTS TO STATETO OEVELUFE AND INPLEMEN } \\
\text { T COMPRETENSIVE SIATE ENERGY CGNSERVATION PLANS } \\
\text { IC RECUCE EY E9 CR MOFE PROJECTEO CONSUMPIION IN } \\
1980\end{array}$ \\
\hline
\end{tabular}


This monthly report lists active prime contracts according to work type. It provides:

- Contractor (1)

- Crganizational Code (2)

- Contract Number (3)

- Task Order or Project Agreement Number (4)

- Award Date (5)

- Total Cumulative obligations (6)

- City and State Work Site (7)

- Contract Status (8)

- Work Category (9)

- Eudget Activity Number (10)

- Contract Type (11)

- Action Category (12)

- Completion Date (13)

- Current FY Obligation (14)

- Total Contract Amount (15)

- Congressional District Code (16)

- Procurement Source (17)

- Prior FY Obligations (18)

- Erief Work Description (19)

See Appendix for coding information. 
IYPE WORK: 7

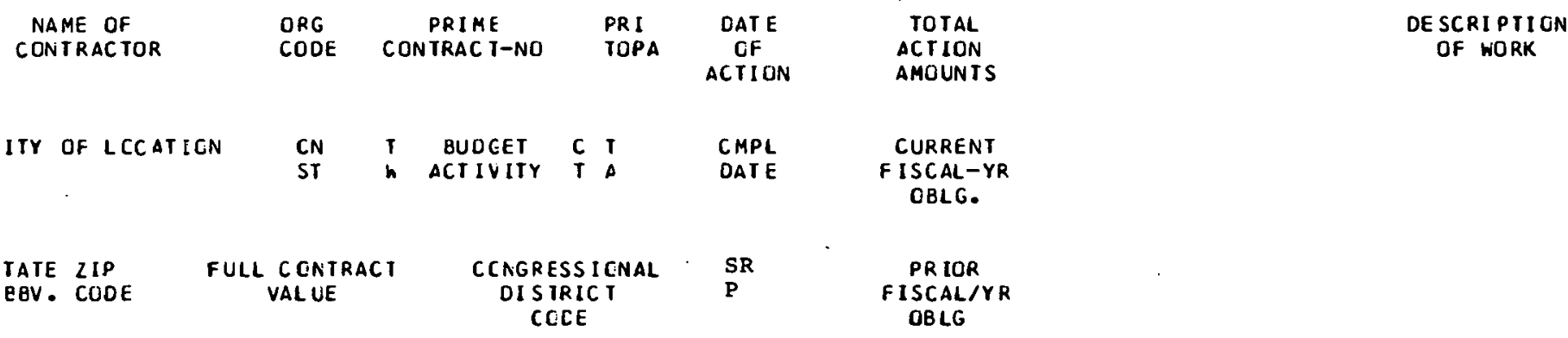

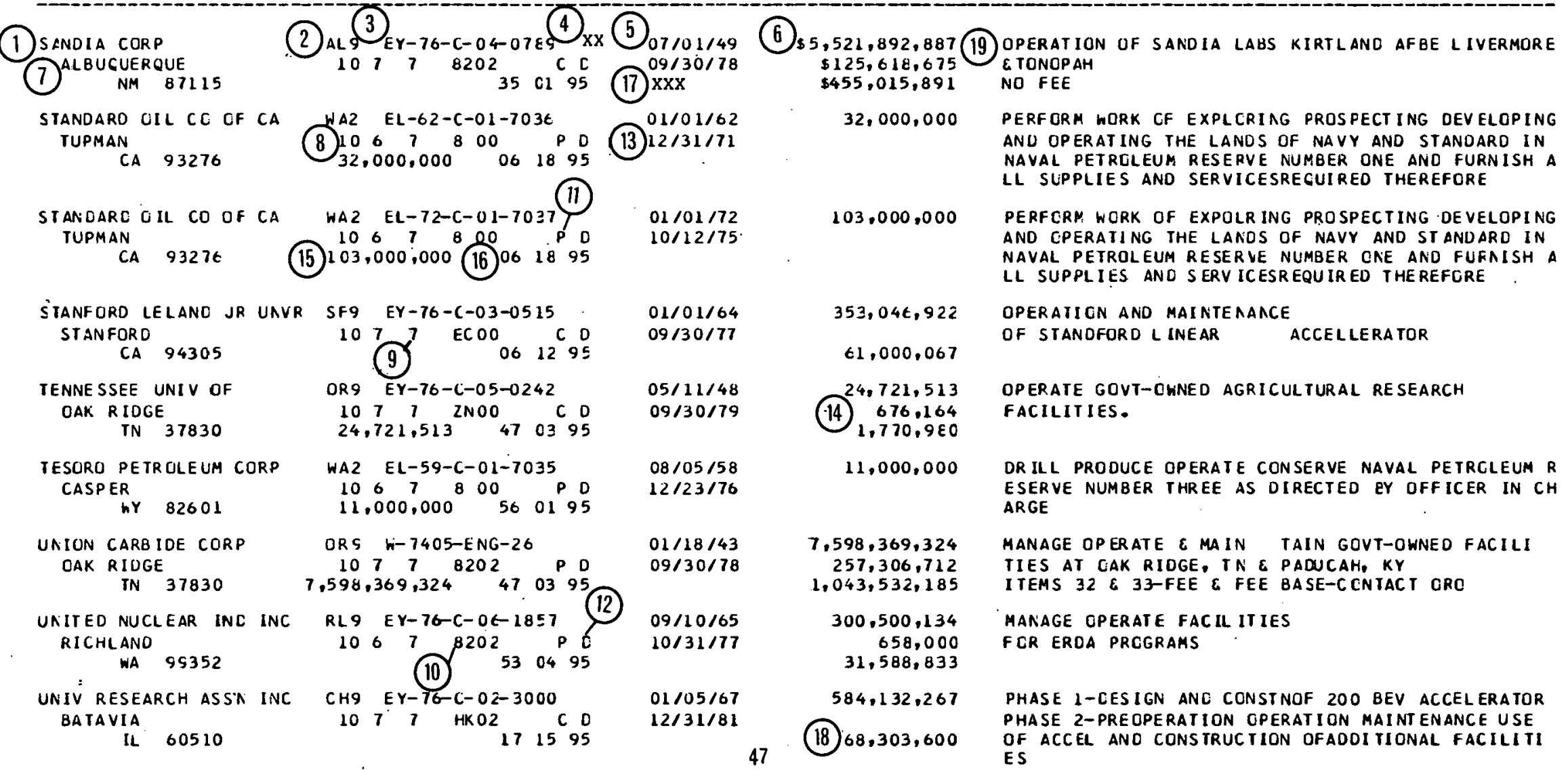




\section{ACT IVE PRIME OPERATING CONTRACTS}

This monthly report lists all active prime operating contracts. It provides:

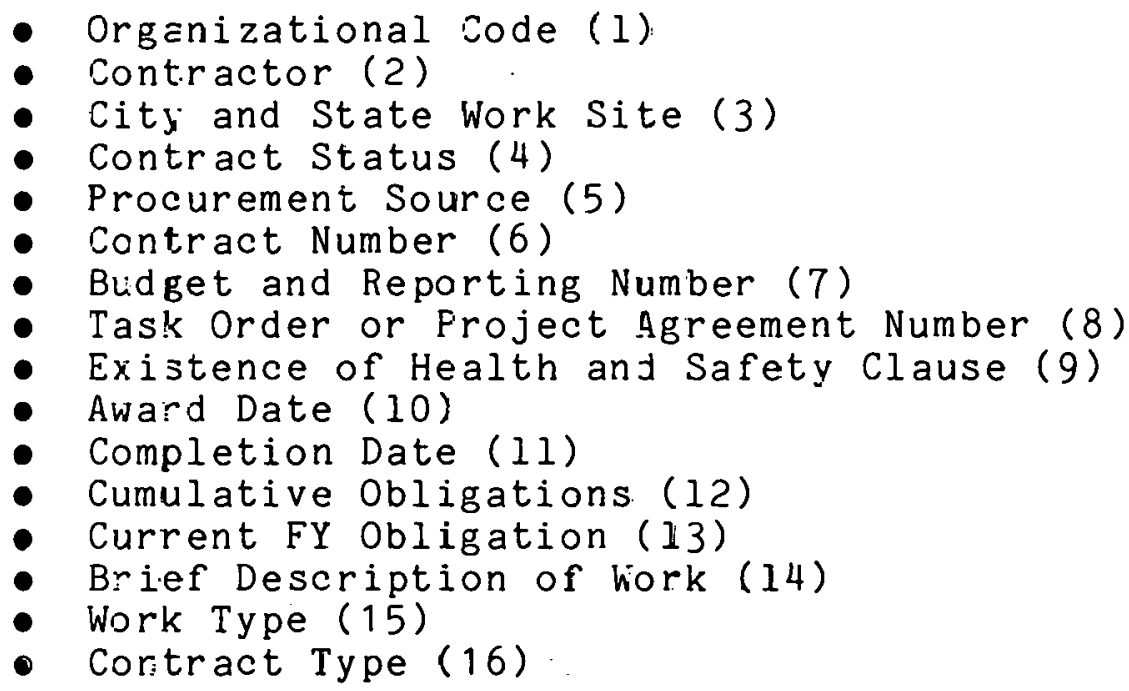

See Appendix for coding information. 
$12 / 31 / 77$

CRG CONE $=\mathrm{CH}$

NAME OF CONTRACTUR

CRG

PR IME
CONTRACT-NO

PRI T DATE

ACTION

TOTAL

ACIICN

AMOUNTS

CITY OF LCCATION

CN BUDGET
ST ACTIVITY

STATE

$\triangle B B V$.

SR

$\begin{array}{ccc}\text { C } & \text { CMPL } & \text { CURRENT } \\ \text { T } & \text { DATE } & \text { FISCAL-YR } \\ & & \text { OQLG. } \\ \text { H } & & \end{array}$

ASSOCIATED UNIVERSITIES UPTCN

(4) 10

$E Y-76-C-02-0016$
$E C O 1$ NY

\section{(10)}

7040846 (2) ausa AND IL

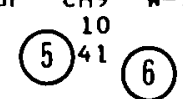

AUA AND CHICAGD. LNIV OF IDAHO FALLS

I $C$

AUA ANC CHICAGO UNIV OF
ARGONNE IL

IOWA STATE UNIV - AMES
AMES AMES

$$
\text { I A }
$$

MATSCO

PHILADELPHIA

PA

PRINCETON UNIV

PR I NCETON

NJ

UNIV RESEARCH ASSN INC C 093080

$-31-109-E N G-38 \quad V 0.1$
KG 10

(6) CH9 H-31-109-ENG-38 10 BAOB

7040846 C 093077

$H-31-109-E N G-38$

092

7040846 c 093077

10 (7) $\mathrm{BAOP}$

CH9 H-7405-ENG-82

10 AKOI C 093078

(15) $\begin{aligned} & 7 \\ & 7\end{aligned} 071576$

30

BA05 $-02-4049$

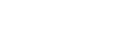

CH9 EY-76-C-02-3073

10 EDOO

(16) $C_{y}^{7} \begin{aligned} & 070151 \\ & 093077\end{aligned}$

7010567

$\mathrm{CH} 9$
10 EY-
$10 \mathrm{ECO}-\mathrm{C}-02-3000$

(9)
$\$ 1,393,155,229$

(12) $2,334,989,210$ (13) 38.755 .709

$2,500,000$

$350,802,827$
DESCRIPTION

OF WORK
OPERATION CF BRODKHAVEN OPERATION OF EROOKHAVEN OTHER ACTIVITIES BA EB RU RH AND UF CONTRACTOR FEE INC RETROYO 070175

OPERATION OF ARCONNE NATIONAL LABORATORY ARGONNE LUCATIDN

14 TO IOENTIFY SEPARATE WORK LOCATION FOR FINANCIAL REPORTS - IOAHO LOCATION

TO ESTABLISH TO/PA FOR PROCUREMENT DIRECTIVES RECEIVED

MULTIPROGRAM NATIONAL LABORATCRY OEALING PRIMARI YIIN PHYSICAL RESEARCH INCLUDING RARE EARTHS $\&$ U $S$ OF AMES RESEARCH REACTOR

OPERATION OF MHO COAL COMBUSTOR AT PERC

RESEARCH AND EXPER IMENTATION IN THE FIELO OF APPLIED PHYSICS

FORMERLY-AT-30-1-1238

PHASE 1-DESIGN AND CONSTNOF 200 BeV ACCELERATCR PHASE 2-PREOP ERATION OPERATION MAINTENANCE USE ACCEL AND CONSTRUCTION ES 


\section{ACTIVE, DOE GRANTS}

This monthly report lists all active prime grants awarded by DOE, ERDA, and AEC. It provides:

- Contractor (1)

- City and State Work Site (2)

- Organizational Code (3)

- Contract Number (4)

- Budget and Reporting Number (5)

- Work Category (6)

- Awarc Date (7)

- Completion Date (8)

- Cumulative Obligations (9)

- Brief Work Description (10)

- Task Order or Project Agreement Number (11)

See Appendix for coding information. 
INAME UF CONTRALTUKN

\section{UR $\bar{G}$}

CODE
PRIME

CONTKRATT-RIE

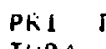

PRi 5

UATE

ACTION

TOTAL

ACTION

AMGUNATS
CESCR IP I ICN

OF WORK

CITY OF LOCATION DUDGET CMPL

STATE

ABBV.

AMES DESIGN CLLLABCKAIIV ALG EG-77-G-04-4130 AIAES

(1) NRCHITECIURAL ALLIIANICE MINNEAFUL. IS $M N$

(4) 111979
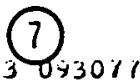

010280 EAOI

af IzCNA UNIV JT

(2) $\mathrm{TP}$.

ARRANSAS STATE JF LIITLE FOCK AR

ARK ANSAS INIIV OF FAYETTEVILLL: $A K$

WA2 EF-77-C-01-2745 3081577
081478

FKO $1-W-78-C-40-0003$ $\mathrm{C} 702$

$8 \quad 101877$ U8317\%

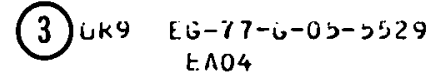

ASSUC GF AMERILAN COLLS OKG EE-77-U-UD-jOLO WASHINGION

$$
\text { (5) } 12106
$$

UR9 $[\dot{v}-77-j-0 j-34]$ EAO 1

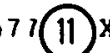

$11) \times 0,00177$ ATLANTA C.

AUBURN UNIV AURUPN

OKG EC-77-G-05-j340

$A L$ EAO 1

BANWELL W'HIIE ARNCLU INL AL9 EG-77-G-04-4139 HANOQVEK EAO1 CALIFORNIA INSI JF TECH SF9 EY-76-Ö-03-LSOS
PASADENA CA 113077

3010377

3093077 113079

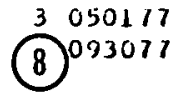

$30 d 1 b 17$ 103077 $09017 \%$ 083177
$\$ 54,500$

19,300

20,000

(10) DEVELCPNEAT CF FICHLUG FLCK LABCFATCRY REACTCR FCR CGAL LIGLEFACIICN

(9) 142,000

12,000

3,500

1,475

1,896

67,716

SUPPLEMEATAL STATE ENERGY CONSERVATICN PRCGRAN A RKANSAS

MIO-SCLTH MORKSHCF CN

ENERGY FROM BIOMASS

NATICAAL CCNFEFENCE OA ENERGY EDUCATION

TOWNES SCHCCL SGLAR

FREJECT ACCESS STAIRCASE

PROCEECINES CIF SLLAR LIVESICCK SHELTERS

CCAFERENCE ON HEATING

DORHITORY DESICA WITH

CCAIINUOLS SOLARIUMS

500,000

INSTITUTICNAL ENERGY 
QUICK LOOK INDEX--ALPHABETIC

This report lists alphabetically all active, inactive and closed prime contracts. It provides:

- Contractor (1)

- Vendor Code (2)

- Contract Number (3)

- Task Order or Project Agreement Number (4)

- Contract Status (5)

- Cumulative Obligations (6)

- Initial or Latest Procurement Action (7)

- Work Category (8)

- Contract Type (9)

- Date of Last Change (10)

- Organizational Code (11)

See Appendix for coding information. 
LV CHS EY-76-S-02-3173

PSN NV9 (4) 015

YUV SF9 AT-04-3-66

$A T$
$A$
$C$
$A$
$A$
$C$
$C$
$C$
$C$
$C$
$C$
$C$
$C$
$C$
$C$
$C$
$C$
$C$
$C$
$C$
$C$
$C$
$C$
$C$
$C$
$C$
$C$
$A$
$C$
$A$
$A$
$A$
$A E$
$A$
$A$
$C$
$C$
$C$
$A$
$C$
$C$
$C$
$C$
$C$
$C$
$A$
$A$
$C$
$C$
$A$
$C$
$A$
$A$
$C$
$C$
$A$

(AMCUNTS IN CCLLARS)

ITEM 26 LAST ITEM 18

(6) 208,589

80,600

211,850

743,668

$1,423,462$

187,000

419,000

422,042

36,994

83,775
57,470

57,470
43,900

43,900
241,027

241,027

83,326

121,623

91,158

300,617

588,300

4,640

4,640
12,702

12,702
26,315

26,315
46,960

46,960
49,278

49,278
7,500

13,590

120,000

5,000

64,000

141,287

250,000

1,680

364,716

248,570

56,266
67,905

67,505
67,066

67,066
463,472

463,472
510,435

510,435
143,452

143,452

158,000

85,900

101,720

159,880

42,061

42,061

33,364

19,028

$4,063,345$

063,345
15,000

(7) 49,100

18,000

271.834

661.731
187.000

419.000

105

36,750

83.775
100

100
43,900

1,795

600

1,348

91.158

3.132

c 2.300

400,000

400,000
3,000

3,000
12,702

12,702
26,315

975
1.550

7,500

13,590

25,000

5,000

32,000

141,287

250,000

840

248,570

56,266

5,796

2,289
8,272

24,015

5,055

26,177

158,000
85,900

2,821

159.880

42.061

32,061

33,364
19,028

15,000
168,500

518

178,434

15,000

(1) $A=A C T I V E$ (10) AE = ACTIVE EXTENSION IN PROCESS (11) AT = ACTIVE - TERMINAL PERQID (12) T = CONPLETEO (30)

ACTICN TYPE 329 THIS PD WRK CON DATE \begin{tabular}{lll}
3 & $S$ & CEC \\
\hline & $S$ & SEP
\end{tabular} CANGED 3 , SAN CHANGED 39P JAN $\begin{array}{ll}8 & F \\ 2 & \text { SEP } \\ 2 & \text { SEP }\end{array}$ $\begin{array}{lll}2 & F & \text { SEP } \\ 2 & F & S E P\end{array}$ 2 F SEP 2 F SEP

4 F SEP

CHANGED 2 F SEP SEP SEP SEP SEP SEP SEP SEP SEP

CHANGED

\footnotetext{
IC = COMPLETED - CONIINGENCY FOR WARRANIY OR CLAIM STILL EXISTS 1311 C = CLOSED-OUT (TC)
} 


\section{QUICK-LOOK INDEX--NUMERIC}

This monthly report lists all active, inactive and closed prime contracts. It provides:

- Organizational Code (1)

- Contract Number (2)

- Task Order or Project Agr aement Number (3)

- Contractor (4)

- Contractor Code (5)

- Status Code (6)

- Cumulative Obligations (7)

- Initial Amount or Latest Action Amount (8)

- Work Category (9)

- Contract Type (10)

- Date of last change (II)

See Appendix for coding information. 
STATUS

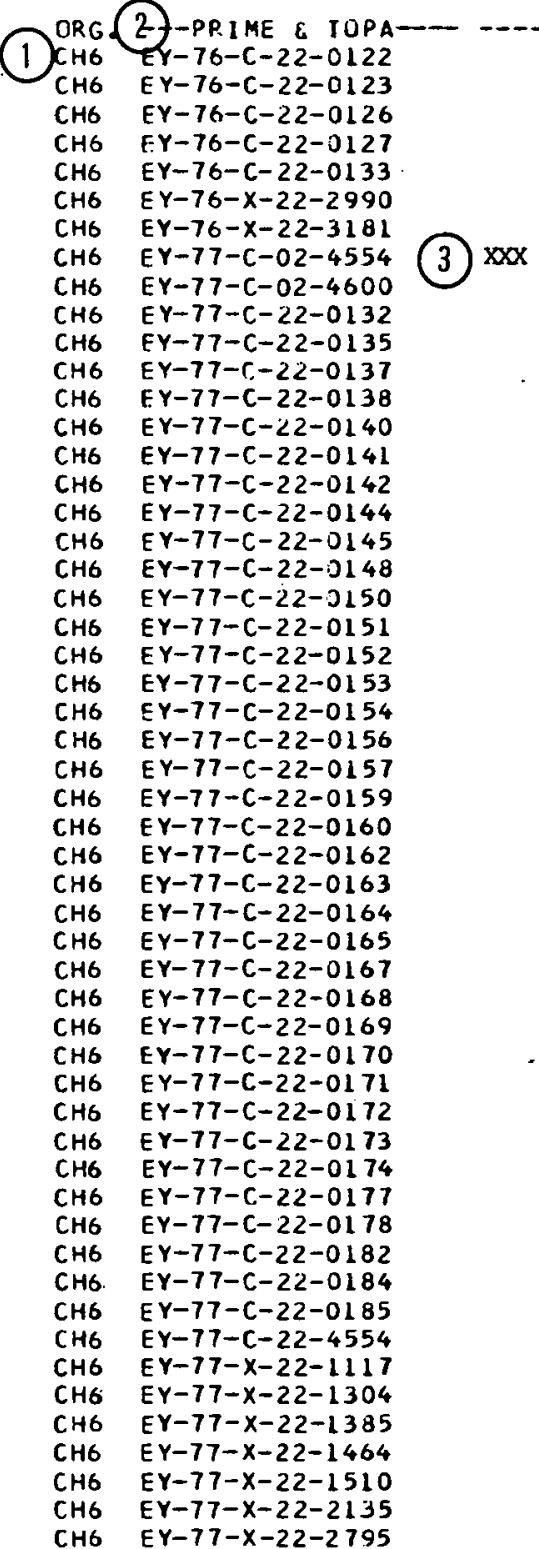

- --VENUOR NAME AND CODE-_- 11 SENECA ENVIRONAENTAL PRO TZO C

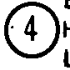

HYORAMET AMERICAN INC LECO CORP

TEWILO INDUSTRIES INC MND $C$

WIDDING F B ASSOCS INC KFO C
FUREST BUILDERS INC

BISSELL ANO COFFEY

AUTOCLAVE ENGINEERS INC XIH AE

AUTOCLAVE ENGINEERS INC XIH A

FISHER SCIENTIFIC CO INC MVV C

LAWRENCE MARIANI SR EUD A

NORHAN JACKSON CONTRACT OUD

HYLAND ELEC SUPPLY CO NLF C
OURIRON CO INC

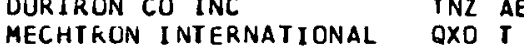

HEAT EXCHANGE $E$ TRANSFER FVO C-6

GENERAL CRYOGENI SS CORP MDI

INDUSTRIAL DISP. SUP CO PMD C

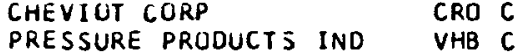

PRESSURE PRODUCTS IND VHB $C$

INTERNATL TEL $\&$ TEL CORP ZJY C

ALBAN LIFT TRUCK INC OZO C

HEAT EXCHANGE \& TRANSFER FVO $C$

GEORGE SIRAKOS YOO $C$

SCALE ELECTRONICS INC VOO

FISHER SCIENTIFIC CO INC MVV

AUTOCLAVE ENGINEERS INC XIH

IONICS INC

TEA T

RAPID ELECTRIC CO INC YVK C

HOFFMAN ENGINEER ING INC LYS AT

COPE MATERIAL HANOLING WSO C

ENPRO INC

NORTHRUP INCORPORATED FSO A

RAMSEY ENGINEERING COMPA XUO C

RAPIO. ELECTRIC CO INC YVK A

NORMAN JACKSON CONTR \#\# C

YALE INDUSTRIAL TRUCKS AHR C

BIRTLEY ENGINEEF.ING CORP LAR A

BALOWIN ELECTRIC CD \# A

CROMPION AND KNOWLES CO

FOREST BUILDEKS INC \$Y AY A

FOREST BUILDERS INC SYS

CHEMICAL DATA SYSTEMS WZO

FISHER SCIENTIFIC CO INC MVV

VIDEO LOGIC CORP COO

LEEDS \& NORTHRUP CO TIF $C$

DIGITAL EQUIPMENT CORP ZRV C
LEEDS \& NORTHRUP CO 5 IF
(AMOUNTS IN OOLLARS)

10,477 THIS PO WRK CON OF 329

10,477

35,900

13,443

14,240
116,446

10,650

6,558

743,000

386,400

122,990

162.350

24,495

21.726
5.356

8,258

8.258
16.652

16.652

41,500

9,162
3,868

23,460

2,807

(7) 24,780

16.200
7,030

7,030

49,072

12,02

34,193

19,525

19,525

21,980
25,469

25,469
12,181

12,181
10,135

10,135
9,160

10,250

16,685

8,000

14,019

13,558

11,391

22,005

20,680

71.600

9,850

743,000

15,488

58.416

42,000
40,525

40,525
107,185

10,504

11,746
10,477

35.900
13.443

13.443
14.240

14,240
116,446

116,446
1,250

6.558

743,000

386.400

122,990

162,350

24,495

$10,8 \in 8$

5,356
8,258

8,258

16,652

41,500

9,162

1.050

2,807

4,320

16,200

7,030

9,655
49,072

168 CHANGED

44- CHANGED

525 CHANGED

21,980 CHANGED

25,469 CHANGED

12,181 CHANGED

10.135 CHANGED

, $l$ EO CHANGED

300 CHANGED

16.685 CHANGED

8,000 CHANGED

14,019 CHANGED

13,958 CHANGED

2,911 CHANGED

9,662 CAANGED

20,680

9,850

743,000

43.000
175

58,416

15.750

5,245

107,185

10,504
11,746

CHANG

CHANGED

CHANGED

CHANGED

CHANGED

CHANGED

CHANGED

CHANGED
632- CHANGED

= CONPLEIEO 1301

TC = COMPLETED - CONTINGENCY FOR HARRANTY OR CLAIM STILL EXISTS 131 C $\mathrm{C}$ = CLOSEO-OUT (7OI 


\section{ALPHABETICAL INDEX}

This monthly report is an alphabetical list of all active and inactive contracts. It provides:

- Contractor (1)

- City and State Work Site (2)

- Cumulative Obligations (3)

- Contract Amount (4)

- Organizational Codes (5)

- Contract Number (6)

- Task Order or Project Agreament Number (7)

- Congressional District Code (8)

- Organizational Code (9)

- Agency Administering the Contract (10)

- Contract Status (11)

- Work Category (12)

- Contract Type (13)

- Award Date (14)

- Completion Date (15)

- Budget and Reporting Number (16)

- Brief Work Description (17)

See Appendix for coding information. 
REPORT NO. H-0402307-021

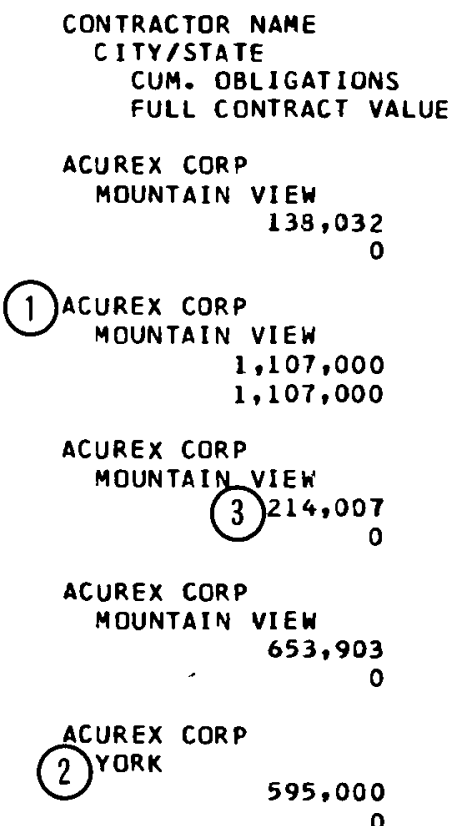

$$
\begin{array}{r}
33,697 \\
0
\end{array}
$$

337,247 338,194

U. S. DEPART MENT OF ENERGY

PROCUREMENT ANO CONTRACTS MANAGEMENT DIRECTORATE CONTRACTS INFORMATION SYSTEM

$$
\text { A LPHA I NDEX }
$$

AS OF DATE

$77 / 12 / 30$

ORG. CONTRACT NO. E TOPA CONG DISTRICT CODE

STATUS

ORIG AHRD CORRESPONDENCE CODE TYPE CONTR BUDG-ACTV

TYPE WORK

EST COMPL

(5)AL9 EG-77-C-04-41217 $\mathrm{xX}$

$A L 960-77-C-04-4159$

CA 3100061295

CA

SF9 EG-77-C-03-1713

$3700 \quad 061295$

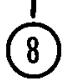

SF9 EY-76-C-03-1218

CA $3700 \quad 061295$

(10) Doe

WA2 EF-77-A-01-2573

PA $9902 \quad 421995$

DOE

SF9 EG-77-A-03-1518

CO $3700 \quad 08 * * 95$

VA EX2 EX-76-X-10-1046

VA $9902 \quad 511095$

DOE

BR1 EX-77-C-65-0050

CO $6070 \quad 080495$ OT HER

DOE

BR1 EX-76-C-65-0004 6070190695

(9) TI HER

SD $6 R 1$ EX-76-C-65-0008

SD $6070 \quad 460295$

OTHER
AC TIVE

RED

CPFF

AC TIVE

RED
CNF

\section{(11) $A C T I V E$ \\ RED \\ $77 / 09 / 26$ \\ $78 / 06 / 26$ \\ EAO 1}

CPFF

(12) ACTIVE

$R E D$

CPFF

ACT IVE

O PGM

(13) $\mathrm{CO}-\mathrm{CP}$

ACTIVE

$R E D$

FFP

CAPL-TERM

MSE'-O

FFP

$77 / 09 / 16$

$78 / 11 / 15$

EAO I

$77 / 09 / 30$

EAO3

DETAILED DESIGN

$N$ AND CHECKOUT OF 150

IRRIGATICN PROJECT

76/03/15 17APPLICATION OF SOLAR ENERGY TO THE SUPPLY OF 77/03/31 17NOUSTRIAL PROCESS HOT WATER EAO1

$76 / 11 / 15$ $78 / 10 / 28$ $\mathrm{BAO} 3$

COOPERAT IVE AGREEMENT TO UNDERTAKE AND MANAGE A DEMO PROJECT TO INSTALL INSTRUMENTATION

$77 / 03 / 01$ $83 / 03 / 01$ EAO 1

\section{ACT IVE}

CONST
FFP

$75 / 07 / 02$ $76 / 09 / 30$ UA 20

ACTIVE

CONST

CONS
FFP

ACT IVE

CONST

FFP

57
$77 / 03 / 31$ $78 / 06 / 01$

800

$75 / 08 / 14$ $77 / 09 / 12$ (16) 800

$76 / 04 / 29$ $77 / 08 / 24$ 800
SOLAR HEATING AND COOLING DEMO PON PROJECT

FURNISHING OF GRAPHICS SUPPLIES DURING FY76 AS

BEAVER CREEK SUBSTATION STAGE 04 ADDITIONS 1 THRU 31

CONSTRUCT HURON SUBSTATION STAGE 10
SOLAR PRODUCTION OF INDUSTRIAL PROCESS STEAM 
This monthly report lists all active and inactive contracts alphabetically within each contract category, in field office sequenze. It. includes:

- Organizational Code (1)

- Contractor (2)

- City and State Work Site (3)

- Cumulative Obligations (4)

- Contract Amount (5)

- Organizational Code (6)

- Contract Number (7)

- Task Order or Project Agreement Number (8)

- Congressional District Code (9)

- Agency Administering the Contract (10)

- Contract Status (1I)

- Contract Type (12)

- Award Date (13)

- Completion Date (14)

- Brief Work Description (15)

- Work Category (16)

See Appendix for coding information. 
REPORT NO. H-0402303-019

CONTRACTCR NAME

CITY/STATE

CUM. OBLIGATIONS

FULL CCNTRACT VALUE

(2) ATCHISEN TOPEKA SF RR AL BUOUER OUE RT NM 780.818

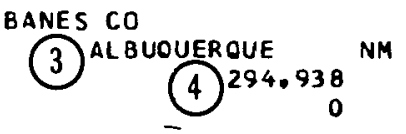

BANES CO

AL BUOUEROUE NM

$$
\text { (5) } 72.192
$$

BANES CO

$$
\begin{gathered}
68,868_{0}^{N M} \\
0
\end{gathered}
$$

BARBER NICHOLS ENG CO ARVACA

$$
19.800^{\circ}
$$

BARBER NICHOLS ENGINEERG ARVADA

$$
\begin{array}{r}
242.958^{\circ} \\
0
\end{array}
$$

$$
\begin{gathered}
\text { BECKETT HARMON ETC } \\
\text { DENVER } \\
16.194 \\
0
\end{gathered}
$$

BERGER ASSOC

COLUMBUS

$$
12.602^{\mathrm{OH}}
$$

BLACK E VEATCH ENGRS

$$
\text { AL BUOUEROUE }
$$

$$
\begin{aligned}
& 1.211 .074 \\
& 1.211 .074
\end{aligned}
$$

BLACK E VEATCH ENISRS

$$
\begin{array}{r}
\text { KANSAS CIIY } \\
157.900 \\
0
\end{array}
$$

ORG CONTRACT NC. E TOPA CONG. DISTRICT CODE CONTRACT ADMIN
(1) ${ }_{3100}^{\mathrm{ELY}} \mathrm{EY}_{350195} \mathrm{C}-04-0829$ (8) $\mathrm{Xx}$ DP

AL9 EY-77-C-04-4100 3100350195

DOE

AL9 EY $-77-C-32-3930$

3100350195 OP DOE

AL9 EY-77-C-32-3938 310350195

(6) DOE

AL9 EG-77-C-04-4162 $3100 \quad 080595$

\section{7}

AL9 EY-76-C-04-3715

$3100 \quad 080595$

$$
\text { DOE (9) }
$$

AL9 EY-76-C- $04-3724$ 3100

$$
\text { DOE }
$$

AL9 EY-77-C-04-3907 3100390895

$$
\text { (10) } \mathrm{DOE}
$$

AL9 EY-76-C-04-3688

$7100 \quad 350195$

$$
\text { DOE }
$$

AL9 EY-76-C-33-0071

$3100 \quad 290595$

MA

\section{STATUS AWARDED \\ TYPE WORK COMPLETED \\ CONTRACT}
ACT IVE
OTHER
$\begin{array}{r}49 / 11 / 01 \\ 78 / 06 / 30\end{array}$ FFP

CONST OPER \& MAINT OF SPUR TRACK SANDIA BASE

ACTIVE CONST FFP

$77 / 09 / 07$ $14)^{78 / 03 / 19}$

CENTRAL RECEIVING AND

ITR I LOVELACE

(11) Active FFP

\section{ACT IVE CONST \\ 12) $F F P$}

AC T IVE

6FP

$\begin{array}{ll}\text { ACTIVE } & 76 / 06 / 08 \\ \text { RED } & 76 / 11 / 01 \\ \text { FFP } & \\ \text { ACTIVE } & 76 / 07 / 12 \\ \text { A-E } & 77 / 12 / 31 \\ \text { FFP } & \\ \text { ACTIVE } & 77 / 05 / 02 \\ \text { A-E } & 79 / 01 / 31 \\ \text { FFP } & \\ & \\ \text { ACTIVE } & 76 / 02 / 12 \\ \text { A-E } & 79 / 02 / 28 \\ \text { FFP } & \\ & \\ \text { CMPL-TERM } & 76 / 08 / 23 \\ \text { A-E } & 78 / 01 / 15 \\ \text { FFP } & \end{array}$

DESIGN E FABRICATIONATING GEOTHERMAL PUMP WASH DESIG DGE-76-54

HOODS SCRUBBERS \& BLDG 123 ROCKY FLATS CARRIER \& DAY INC

\section{PLANT}

UPGRADE ORAINAGE SM-PP

HILL AT MOUND LAB

5MW SOLAR THERMAL TEST FACILITY AT SANDIA LABS DSE-76-39 S35 HASH AUTHOR IZATION NO

ARCHITECT-ENGINEERING SERVICES IN CONNECTION WITH FIREWALL CONSTRUCTION 
This monthly index lists all active prime contracts by work category in field office sequence. It includes:

- Contractor (1)

- City and State Work Site (2)

- Cumulative Obligations (3)

- Contract Amount (4)

- Organizational Code (5)

- Contract Number (6)

- Task Order or Project Agreement Number (7)

- Congressional District Code (8)

- Organizational Code (9)

- Agency Administering the contract (10)

- Contract Status (11)

- Procurement Source (12)

- Contract Type (13)

- Award Date (14)

- Completion Date (15)

- Brief Work Description (16)

See Appendix for coding information. 
REPORT NO. H-0402309-022

ALBUQUERQUE OPERATIONS OFFICE

$$
\begin{gathered}
\text { CONTRACIS INEORMATION SYSTEM } \\
\text { T P E D F W OR K I N DEX } \\
\text { RESEARCH AND DEVELOPMENT }
\end{gathered}
$$

ORG

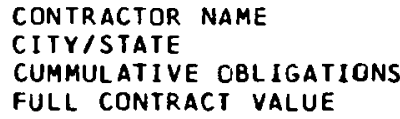

(1) SOLAR KING CORP

$$
\begin{array}{r}
\text { RENO } \\
41,000 \\
0
\end{array}
$$

SOLAR ROOM CO INC TAOS

$$
25,875
$$

\section{SOLARON CORP}

(2) DENVER

$$
99.495
$$

SOLATION PRODUCTS INC

$$
\text { YORK (3) } 98,899
$$

SOUTHWEST RESEARCH INST SAN ANTONIO

$$
\begin{array}{r}
258,699 \\
0
\end{array}
$$

SOUTHWESTERN PBLC SVS AMAR ILLO

\section{IX}

$$
\begin{aligned}
& 400,000 \\
& 400,000
\end{aligned}
$$

SPECTROLAB INC

$$
\text { SYLMAR }
$$

$$
\text { (4) } 561,016
$$

STANFORD LELAND JR UNVR STANFORD

$$
250.000
$$

STANFORD LELAND JR UNVR STANFORD

$$
\begin{array}{r}
75,000 \\
0
\end{array}
$$

STANFORD LELAND JR UNVR STANFORD

$$
69,965 \text { CA }
$$

ORG CONTRACT NO. E TOPA CORRESPONDENCE CODE CONTRACT ADMIN

3100320195 DOE

AL9 EG-77-G-04-4129

3100350195

DOE

AL9 EY-77-C-04-3713 3100080195

$9 \mathrm{DXE}_{\mathrm{XXX}}$

AL9 EG-77-G-04-4137 $3100 \quad 421995$

\section{(6)}

AL 9 EY-76-C-04-3684

$3100 \quad 482195$

DOE

AL9 EN-77-C-04-3970

$3100 \quad 481395$

(10) DOE

AL9 EY-76-C-04-2748

3100060595

DOE

$\begin{array}{ll}\text { AL } 9 & E Y-76-S-04-3570 \\ 3100 & 061295\end{array}$

DOE

AL9 EY-76-S-04-3580

$3100 \quad 061295$

DOE

AL9 EY-76-S-04-3605

$3100 \quad 061295$

DOE 18
(5) AL $9 \quad E Y-76-C-04-3751$

STATUS

AHARDED

SRCE PRDC COMPLETED

CONTRACT

ACTIVE $\quad 76 / 06 / 15$

SMALL. BUS 76/12/31

C-SHR

ACTIVE

12) SMALL EUS

GRAN T

$77 / 09 / 30$

$78 / 09 / 30$

AC T I V

(4)

ACTIVE

FFP
(11) ACTIVE SMALL BUS 77/09/30 SMALL BUS $1578 / 09 / 29$
GRANI

$\begin{array}{ll}\text { ACIIVE } & 76 / 09 / 01 \\ \text { RESCH LAB } & 77 / 11 / 30 \\ \text { CPFF } & \end{array}$

CPFF

\section{ACTIVE $\quad 77 / 03 / 01$} BIG BUS $77111 / 30$ (13) $\mathrm{CNF}$

CMPL-TERM 75/06/16 BIG BUS $77 / 05 / 31$

CPFF $\begin{array}{ll}\text { ACTIVE } & 75 / 05 / 01 \\ \text { UNIV/COL } & 77 / 09 / 30\end{array}$ UNIVICOL SRSA

ACTIVE $75 / 06 / 15$ UNIV/COL $\quad 77 / 12 / 31$ SR SA

\section{CMPL-TERM} UNIVIEOL

$75 / 06 / 15$ SRSA

61

RESEARCH \& DEVELOPMENT MEDIUM GRADE THERMAL MACHINE

PERFORMANCE ANALYSIS

INFLATED HYBRIO SOLAR

A DOUBLE POLYETHYLENE SPACE HEATING SYSTEMS

16 improvement of solar

AIR HEATERS

AS OF DATE $77 / 12 / 30$ PROCESSING DATE 78/01/23 


\section{STATISTICS REPORT}

This monthly report contains statistical data for each field office and Headquarters (Procuremen= Operations).

It includes:

- Field Office's Total Active Contracts (1)

- DOE's Total Active Contracts (2)

- Classification (3)

- Work. Category (4)

- Acticn Category (5)

- Procurement Type (6)

- Latest Award Amount (7)

- Procurement Source (8)

- Contract Type (9)

- Cumulative Obljgations (10)

- Required Performance Reports (11)

- Contract Status (12)

- Small Business Set-Aside Program (13)

- Minority Firm (14).

- Existence of Safety and Health Clause (15)

- Cost-Pricing Data (16)

- Small Business 8(a) Program (17)

- Labor Surplus Area (18)

See Appendix for coding information. 
CLASSIFICATION

(3) UNCL CONF SECRET T.S. UNKN

TYPE OF MORK

TYPE OF ACTION

(4)

$\triangle E E$ CON

CONSTR

(5) LETTER DEFINITIVE P.0. INTR AGREE UNKN

TYPE OF PROCUREMENT

INTRA-CO GSA-F

SA-FS

GSA-LSC OTH-GOV ACVFRT

2

24

N-COMPT N-OTHR

UNS PROP

SVS
2

LATEST AHARD AMT

(7) UN 9.9 10-24.9 $25-49.9$ 50-99.9

$3 \quad \begin{array}{rrr}2 & 225-999.9\end{array}$

PROCUREMENT SOURCE (8) GOVT DOE OFA SELG F-GOV S-BUS B-BUS F-COM UNIV STLAB NPLAB HOSP IRLAB DTHER UNKN

TYPE OF CONTRACT

(9) 21 FPP FPI FPR CNF

2 CPIF CPFF

CPAF TEM

LH SRSA GRANT

NF CS CA
5

TOTAL AWARD AMT

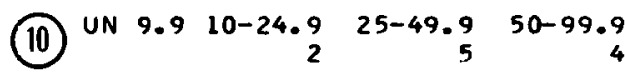

$100-224$

$225-999.9 \quad 1000 \quad U P$

(THOUSANOS OF DOLLARS)

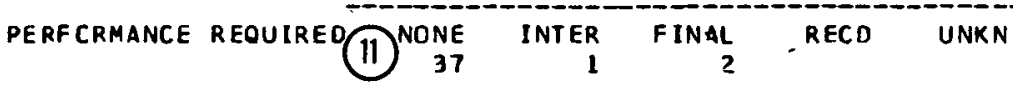

STATUS OF CONTRACT (12)

5 BUS SET-ASIDE PGM (13) 10 NES 26 UNKN

KNOWN MINOR FIRM

(14) YES $\begin{array}{ccr}\text { NO } & \text { INKN } \\ & 1 & 45\end{array}$

SAFETY-HEALTY

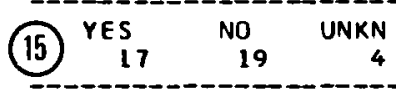

COST-PRICING CATA WAIVEO OBT-AU-WAIVEO

(16) WAIVED OBT-AU-WAIV

OBT-AU-MADE

NOT REQ UNKN

(17) YES ${ }_{36}$ UNKN

SM BUS 8 PROGRAM (17) YES NO UNKN

LABOR SURPLUS AREA (18) OERTFO OTH LS AREA NON-PERF NOT LS UNKN

$\begin{array}{lccc}\text { AREA } & \text { NON-PERF } & \text { NOT LS } & \text { UNKN } \\ 1 & 22 & 13 & 4\end{array}$

ALBUOUERQUE OPERATNS OFF

40 COMPLETED PRIME CONTRACTS
15

4




\section{CONTRACTOR RANKING BY CURRENT FISCAL YEAR OB:IGATIONS}

This monthly report lists the 101 contractors with the largest current FY obligations in descending order. It also provides:

- Contractor's Address (1)

- Contract Number (2)

- Task Order or Project Agreement Number (3)

- Prior FY Obligations (4)

- Cumulative Obligations (5)

- Program Area (6)

- Brief Work Description (7)

See Appendix for codinf information. 


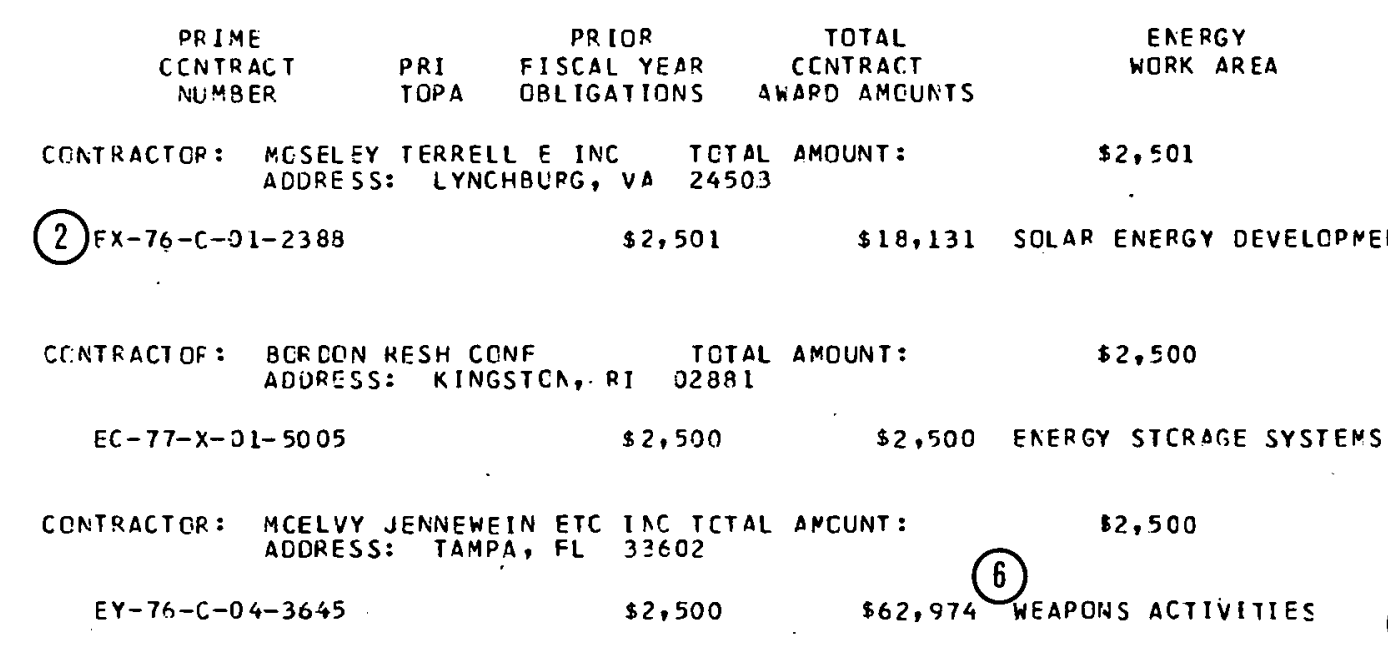

(1)

Cointractor: angor

AVON ELECTRICAL SUPPLIES TOTAL ANOUNT: AODRESS: HAUPPAIJEE LONG ISLAND, NY 11787 $E Y-77-C-21-81 \in 23 \times X X$

52,405

CONTRACTOF: : CLEAN SWEEP INC
AUDRESS: OAK RICGE, TR 37830

$E Y-76-C-05-5156$

$\$ 2,380$

(5) $\$ 85,760$

INFORPATICN SERVICES

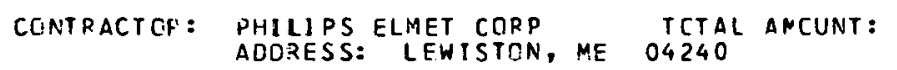

52,321

$E Y-77-C-05-5326$

$\$ 2,321$

$\$ 2,321$

NUCL EAR MAIERIALS

CONTRACTOF: AMERICAN INSTRUMENT CO TETAL AMOUNT:
ACURESS: SILVER SPRINGS, MD $20 \subseteq 10$

EY $-76-X-21-3094$

$\$ 2,134$

$\$ 17,500$

COAL

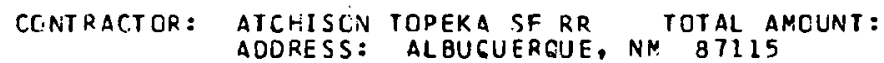

1,901
$\$ 2,1.34$
$\$ 1.901$
$\$ 780,399$
EY-76-C-04-0829

$\$ 780.399 \mathrm{~min}$

INSTALL CPERATE AND MAINTAIN A SPACE AND WATER HEATING SOLAR SYSTEN CN AN OFFICE BUILCING LYNCHBURE VA
DESCRIPTION
CF

\section{CONDUCT $\triangle$ FIVE DAY CCNFERENCE ON}

FLECTROCHEMISTRY IN SAMTA BARBARA CA

(7) FIRE SAF ITY E CFERATIGASCONOITICNS JENNEWEIY STEFANY E HCWARE ARCFITECTS PLANELV JENNEWEI I STEFAAY \& HGWARC ARCHITECTS PLANNERS INC

ELECTRICAL SUPPLIES PROVIDE BUILDING CLEAN-ING SERVICES E SUPPLIESIN
ACCORD WITHSPECS ATFCUR ERDA RUILCINGS

ESTIMATED ANNUAL REGUIFEMENTS FOR WELCING PARTSS ITEMS

SPECTROPHCTOFLLCROMETERWITH NC-ZCNE HCUSINE NAGNETIC ARC STABIL IZER ANCG-FIXEC SPEED SCANASSEFBLY ANC CORRECTELSPECTRA ONC X-
RECORDER

CONST OPER E MAINT OF SPUR TRACK SANOIA BASE

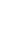


SUMMARY OF TOP 101 CONTRACTORS FOR FISCAL YEAR

This monthly report lists in descending order the top 101 contractors, who have the most finds obligated for the current fiscal year. 


$$
\begin{aligned}
& \text { CONTRACTCR } \\
& \text { TOTAL } \\
& \text { CURRENT FY } \\
& \text { OBLIGATIONS }
\end{aligned}
$$

IOWA STATE UNIV - AMES

$\$ 13,653,015$

ROCHESTER UNIVERSITY OF

$\$ 12,517,279$

$\$ 12,436,419$

TALLEY FRAC CCRP

$\$ 11,849,919$

PARSONS RALPH M COMPANY

UNITED TECHNOLCGIES CORP

$\$ 11,583,657$

$\$ 11,357,000$

$\$ 10,000,000$

WALLACE / BROWN-OLDS/HOHAR

$\$ 9,705,069$

$\$ 9,637,900$

NAIIONAL RUREAU SIANDARD

$\$ 9,080,700$

$\$ 9,029,065$

$\$ 8,970,082$

$\$ 8,830,370$

$\$ 8,680,074$

$\$ 8,028,256$

$\$ 7,650,000$

$\$ 7,417,505$

$\$ 7,201,773$

$\$ 7,198,267$

$\$ 6,945,171$

$\$ 6,840,302$

$\$ 6,676,377$

$\$ 6,664,297$

$\$ 6,292,000$

$\$ 6,264,500$

$\$ 6,219,730$

$\$ 6,187,000$ 
THIS PAGE

\section{WAS INTENTIONALLY LEFT BLANK}




\section{SPECIAL REPORTS}

The public can request some special reports that are already available for ten cents a page to cover the cost of reproduction. However, we cannot provide reports requiring differing formats or other data elements than those contained in the reports in this publication. If individualized programming is necessary to compile a special report, there are additional charges of $\$ 7.98$ an hour plus computer time charge. These fees are subject to change.

Requests for special reports should be addressed to:

Data Management and Analysis Branch Procurement Management Systems and Analysis Division U.S. Department of Energy Washington, DC 20545 
THIS PAGE

WAS INTENTIONALLY

LEFT BLANK 
Region

I

Harold J. Keohane

Analex Building, Room 700

150 Causeway Street

Boston, Massachusetts 02114

I I

Robert A. Low

26 Federal Plaza

Room 3206

New York, New York 10007

III Bernard Snyder (Acting)

1421 rherry Street

10th Floor

Philadelphia, Pennsylvania 19102

IV

Roy Pettit (Acting)

1655 Peachtree Street, NE.

8th Floor

Atlanta, Georgia 30309

V Robert H. Bauer

175 West Jackson Boulevard

Room A-333

Chicago, Illinois 60604

VI Curtis E. Carlson, Jr.

P.0. Box 35228

2626 West Mockingbird Lane

Dallas, Texas 7.5235

VII James R. Newman

324 East 11 th Street

Kansas City, Missouri 64106

VIII Charles P. Netzger

P.0. Box 26247 - Belmar Branch

1075 South Yukon Street

Lakewood, Colorado 80226

IX William C. Arntz

111 Pine Street

Third Floor

San Franciso, California 94111

$X$ Jack B. Robertson

1992 Federal Building

915 Second Avenue

Seattle, Washington 98174
Commercial No. FTS No.

$617-223-3701 \quad 223-3701$

$212-264-1021 \quad 264-1021$

$215-597-3890 \quad 597-3890$

$404-881-2837 \quad 257-2837$

$312-353-0540 \quad 353-0540$

214-749-7345 749-7345

$816-374-2061 \quad 758-2061$

$303-234-2420 \quad 234-2420$

$415-556-7216 \quad 556-7216$

$206-442-7280 \quad 399-7280$ 
THIS PAGE

\section{WAS INTENTIONALLY LEFT BLANK}




\section{Alabama}

David Dyer

Director, Al abama Energy

Extension Service

Auburn University

Auburn, Alabama 36830

(205) $826-4576$

Connecticut

Bradford S. Chase

Director, Connecticut Energy

Extension Service

Office of Policy and

Management

$20 \mathrm{Gr}$ and Street

Hartford, Connecticut 06115

(203) 566-5803.

\section{Michigan}

Dennis Sykes

Director, Michigan Energy

Extension Service

209 Holister Building

Lansing, Michigan 48933

(517) 373-0480

New Mexico

Frank Marquez

Director, New Mexico Energy

Extension Service

P.0. Box 00

Santa Fe, New Mexico 87501

(505) $827-2472$

Pennsylvania

William B. Harral

Governor's Energy Council

State Street Bldg., 12th Floor

Harrisburg, Pennsylvania 17101

(717) $787-9749$ 


\section{Tennessee}

Donald R. Pitts

Director, Tennessee Energy

Extension Service

250 Capitol Hill Building

Nashville, Tennessee 37219

(615) 74l-2994

\section{Texas}

Stephen Riter

Texas A\&M University

College Station, Texas 77849

(713) 845-8025

\section{Washington}

Lloyd Craine

Program Director, Washington

Energy Extension Service

Washington State University

Pullman, Washington 99164

(509) 335-2531

\section{Wisconsin}

William Bernhagen

Energy Programs Coordinator

University of Wisconsin

Extension

437 North Lake Street

Madison, Wisconsin 53706

(608) 263-1562

Wyoming

Sam D. Hakes

Director, Wyoming Energy

Extension Service

University Station

Box 3295

Laramie, Wyoming 82071

(307) 766-4253 


\begin{tabular}{|c|c|c|c|c|c|}
\hline & Fr & TI TLE & & FY & TITLE \\
\hline$\triangle D$ D. & 79 & ENERGY SUPPLY-RESEARCH \& TECHNOLCGY DEV & ADO1 & 78 & THERMAL APPLICATIONS \\
\hline AAUO & 78 & COAL & $\triangle D O 2$ & 78 & TECHNOLOGY SUPPORT AND UT ILIZATICN \\
\hline$A A D L$ & 78 & NINES RESEARCH AND DEVELOPMENT & $\triangle D 03$ & 78 & SOLAR ELECTRIC APPLICATIONS \\
\hline$A A \cup ?$ & 78 & LI QUEFACTION & $\triangle E O D$ & 78 & GECTHERMAL \\
\hline$\triangle A U_{3}$ & 78 & GASIFICATICN & $A E O l$ & 78 & ENGINEERING RESEARCH ANC OEVELOPMENT \\
\hline$A A B) 4$ & 78 & ADVANCED POWER SYSTEMS & $A E U 2$ & 78 & RESOURCE EXPLORATION AND ASSESSMENT \\
\hline $4 A^{\prime} 5$ & 78 & CIRECT COMBUSTICN & AEO3 & 78 & HYOROTHERMAL TECHNOLOGY APPLICATIONS \\
\hline AAO6 & 78 & ADVANCEO KESEARCH AND SUPPORTING, TECH & $A E O 4$ & 78 & ADVANCED TECHNOLOGY APPLICATIONS \\
\hline AAOT & 78 & MAGNE TOHYDREOYNAMICS & AES 5 & 78 & UT IL IZATION. EXPER IMENTS \\
\hline$A B 00$ & 78 & PETROLEUM & $\triangle E 06$ & 78 . & ENVIRONMENTAL CONTROL $\varepsilon$ INSTITUTICNAL \\
\hline$\triangle B O 1$ & 78 & ENHANCED OIL RECOVERY & AFDO & 78 & FUSION \\
\hline$A B D 2$ & 78 & CIL FRCM OIL SHALE & AFOL & 78 & MAGNETIC FUSION \\
\hline$A B \cup 3$ & 78 & DRILLING AND OFFSHORE TECHNOLOGY & $A S 00$ & 78 & FISSION \\
\hline 180.4 & 78 & REFINING CHARACTER IZATICN \& UTILIZATION & AGOl & $\mathbf{8}$ & URANIUM RESDURCE ASSESSNENT \\
\hline $4(00)$ & 78 & GAS & $A G O 2$ & 78 & FUEL CYCLE RESEARCH AND DEVELCPMENT \\
\hline$A C O L$ & 78 & ENHANCED GAS RECOVERY & $\Delta G \Omega_{3}$ & 18 & BREEDER REACTOR \\
\hline $\mathrm{ACO} 2$ & 78 & GAS FROM OIL SHALE & $A G 04$ & 73 & NUCLEAR RESEARCH AND APFLICATIONS \\
\hline$\triangle 00:$ & 78 & SOLAR & $A G 05$ & 73 & LIGHT WATER REACTOR FACILITIES \\
\hline
\end{tabular}




\begin{tabular}{|c|c|c|}
\hline & FY & TITLE \\
\hline$\Delta G_{6} 6$ & 78 & INTERNATIONAL SPENT FUEL DISPJSITION \\
\hline$\triangle H O O$ & 78 & HYOROELECTRIC \\
\hline AHOI & 78 & LDW HEAD HYDROELECTRIC DEVELCPIMENT \\
\hline AJOS & 73 & BI GMASS \\
\hline nJol & 78 & FUELS FQOM BIOMASS \\
\hline$\triangle K O O$. & 7.8 & MULTI-RESDURCE \\
\hline$\Delta K \cap I$ & 78 & BASIC ENERGY SCIENCES \\
\hline AKO9 & 78 & USED NUCLEAR EQUIPMENT GRANIS (NONFUNCI) \\
\hline \multirow[t]{2}{*}{ P Di) } & 77 & FOSSIL ENEP.GY DEVELCPMENT \\
\hline & 78 & ENEPSY SUPPLY PROOUCTION DEME \& DISTRIB \\
\hline \multirow[t]{2}{*}{$B \triangle 00$} & 77 & COAL \\
\hline & 78 & COAL \\
\hline \multirow[t]{2}{*}{8401} & 77 & LIGJEF ACTION \\
\hline & 78 & COAL UTILIZATION, INDUSTRIAL \\
\hline \multirow[t]{2}{*}{$\mathrm{BAO} 2$} & 77 & HIGH-BTU GASIFICATION \\
\hline & 78 & COAL UTILIZATION, UTILITY \\
\hline \multirow[t]{2}{*}{$\mathrm{BAO} 3$} & 77 & LOK-BTU GASIFICATICN \\
\hline & 78 & COAL LGAN GUARANTEE PROGRAM \\
\hline 8404 & 77 & ADVANCED POWER SYSTEMS \\
\hline BAO5 & 77 & CIRECT COMBUSTION \\
\hline
\end{tabular}

3407 77 DENONSTRATION PLANTS

BADB 77 MAGNETOHYORDOYNAMICS

BBO0 77 PETROLEUM AND NATURAL GAS

78 PETR.OLEUM

BBOL 77 GAS AND OIL EXTRACTION

78 NAVAL PETROLEUM AND SHALE RESEP.VES

BBO2 77 SUPPORTIING RESEARCL

BCUO 77 IN SITU TECHNOLOGY PROGRAM

BCOL 77 OIL SHALE

BCO2 77 IN SITU COAL GASIFICATION

BCO3 77 SUPPORTING RESEARCH

BDOO 78 SCLAR

BOD1 78 HEATING ANE COOLING DEMCNSTRATION PROG

BDO2 78 FEDERAL BUILDINGS SOLAR PROGRAM

BD03 78 SOLAR COMMERCIALIZATION

BEOO 78 GECTHERMAL

BEO 18 GECTHERMAL RESOURCES DEVELOPMENT FUND 


\begin{tabular}{|c|c|c|c|c|c|}
\hline & FY & TITLE & & FY & TITLE \\
\hline 8500 & 78 & FISSO'v & $\mathrm{C} 104$ & 78 & CONSUMER OFFICES \\
\hline 8601 & 78 & URANIJM FNRICHMENT ACTIVITIES & C 105 & 78 & RATE REFORM \\
\hline $\operatorname{sgo} 2$ & 78 & URANIUM RESOURCE ASSESSNENT & $\left(20^{\circ}\right)$ & 78 & PESIDENTIAL \\
\hline$B K i) !$ & 78 & MULTI-RESOURCE & $\mathrm{C} 201$ & 78 & BUILDINGS AND COMMUNITY SYSTEMS \\
\hline BKul & 78 & ELECTRICITY & 0202 & 78 & WEATHER IZATION \\
\hline$B K D 2$ & 78 & AL TERNATE FUELS DENO PRCGRAM & $\mathrm{C} 203$ & 78 & APPLIANCES \\
\hline$B K \cup 3$ & 79 & FEDERAL LEASING, & C.204 & 78 & UTILITY INSULATION SERVICE \\
\hline 81.00 & 73 & POWER MAEKETING & $C 205$ & 78 & CTHER RESIDENTIAL \\
\hline BLOL & 78 & ALASKA PGWER ADMINISTRATIGN & $c 300$ & 78 & RESI DENTIAL/COMMERCIAL \\
\hline$B L 02$ & 78 & BONNEVILLE POWER ADMINI STRATION & $c 301$ & 78 & BUILDINGS AND COMMUNITY SYSTEMS \\
\hline BLO3 & 78 & RECLAMATION POWER ACMINISTRATION & (30). & 78 & OTHFR RESI CFNT IAL/CCMMERCIAL \\
\hline BLO4 & 78 & SGUTHWESTERN POWER ADMIMISTRATION & $C 303$ & 78 & $\triangle P P L I A N C E$ \\
\hline $8 L 05$ & 78 & SOUTHEASTERN PDWER ADMINISTRATION & 6.324 & 78 & UT ILITY INSULATION SERVICE \\
\hline$C 00$ & 78 & CONSERVATION & $C 305$ & 78 & WFA THER IZATION \\
\hline C100 & 78 & UTILITIES & $(4) 0$ & 78 & INDUSTRIAL \\
\hline $\mathrm{C} 101$ & 78 & ELECTRIC ENERGY SYSTEMS & $(4) 1$ & 78 & INDUSSTRY \\
\hline$C 102$ & 78 & UTILITIES DEMONSTRAIIGN & $(4) 2$ & 78 & INDUSTRIAL MONITOR ING \\
\hline C 193 & 78 & DISTRICT HEATING & $C 403$ & 78 & COGENERATION \\
\hline
\end{tabular}




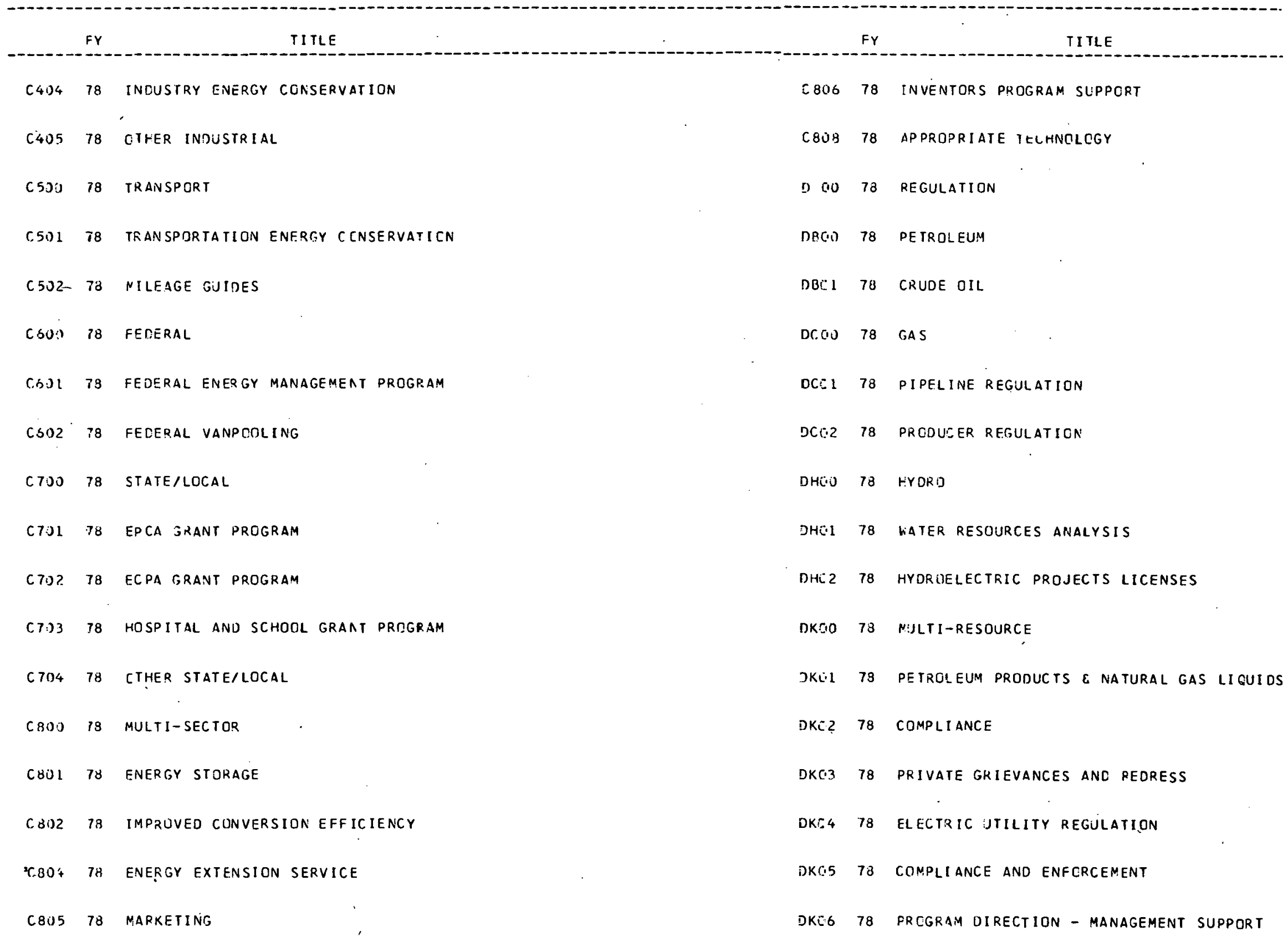




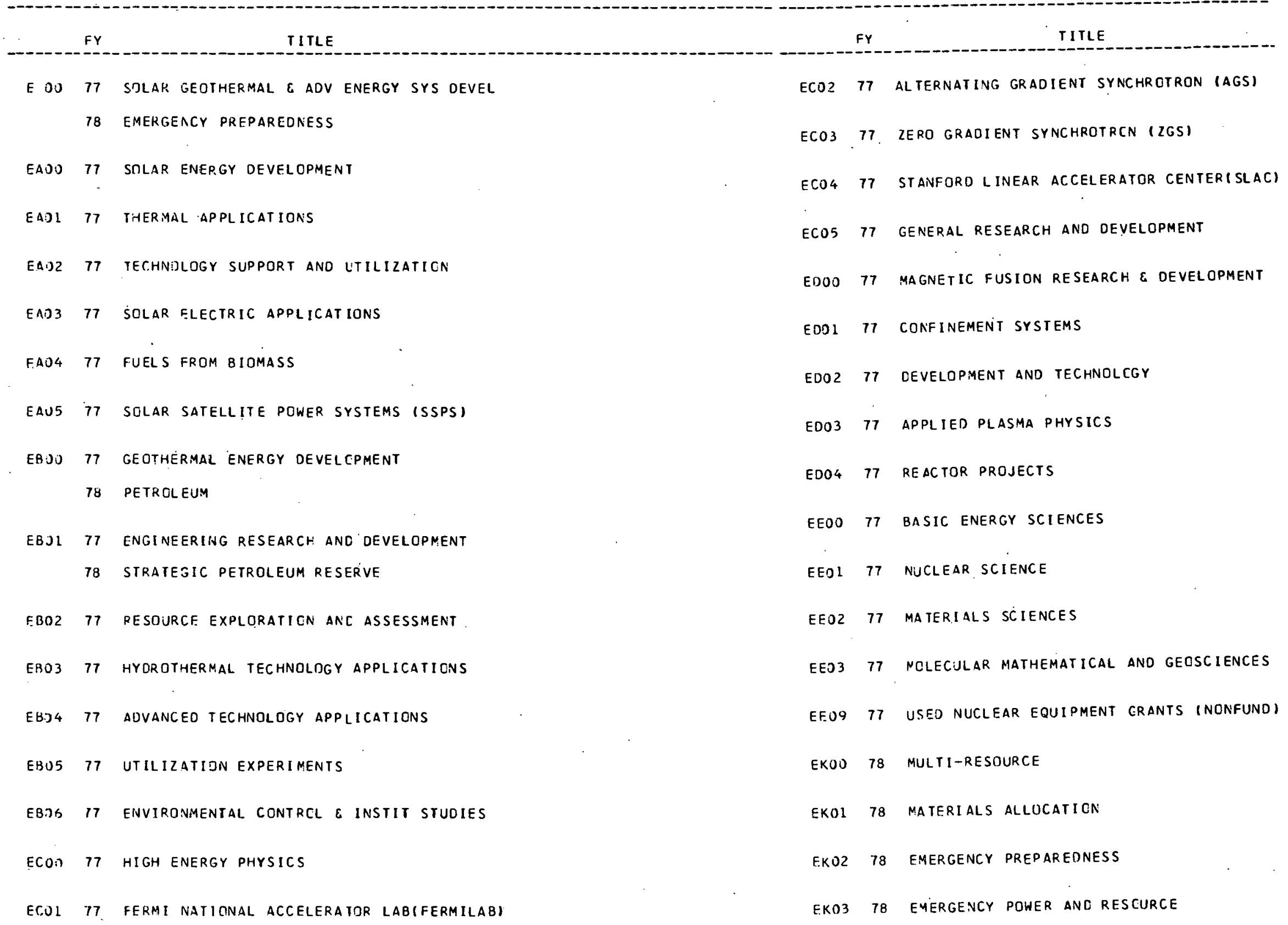




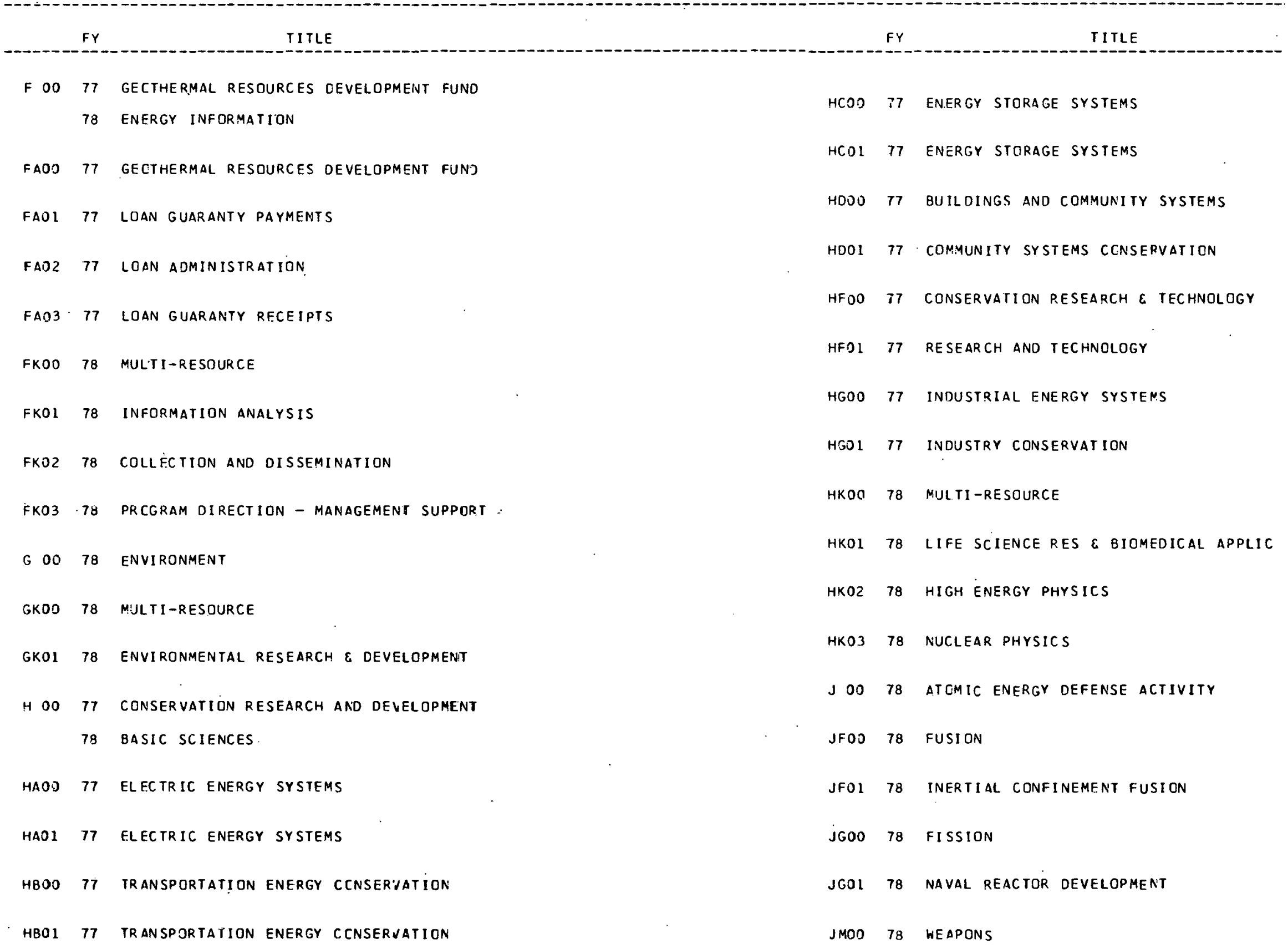




\begin{tabular}{|c|c|c|c|c|c|}
\hline & FY & TITLE & & FY & TITLE \\
\hline JMiJl 1 & 78 & REAPONS ACTIVITIES & $\mathrm{KHOL}$ & 77 & SUBMARINE PROPULSION REACTORS \\
\hline J:M02 & 78 & INTELL IGENCE AND ARMS CCNTROL & $\mathrm{KHOL}$ & 77 & SUSPFACE SHIP PROPULSION REACTORS \\
\hline$J M 33$ & 78 & SPECIAL MATERIALS PRODUCTION & $\mathrm{KHO} 3$. & 77 & SUPPORTING RESEARCH \& OEVELCPMENT \\
\hline 51904 & 78 & NUCLEAR MATERIALS SECURITY \& SAFEGUARDS & KJoO & 77 & NUCLEAR RFSEARCH AND APPLICATIONS \\
\hline $\mathrm{K}$ oO & 77 & NUCLEAR ENERGY DEVELOPMENT & KJOI & 77 & KATER COOLED BREEDER REACTORS \\
\hline & 78 & POLICY AND MANAGEMENT & $\mathrm{KJ} 02$ & 77 & GAS CODLED THERMAL REACTORS \\
\hline$K 600$ & 77 & LIGUID METAL FAST BREEOER REACTOR-LMFBR & $\mathrm{KJO} 3$ & 77 & GAS COOLED FAST BREEDER REACTORS (GCFR) \\
\hline KGOl & 77 & LARGE PLANTS & $\mathrm{KJ} 04$ & 77 & MOLTEN SALT BREEDER P.EACTGRS (MSBR) \\
\hline KG02 & 77 & CLINCH RIIVER BREEDER REACTOR PLANT PROJ & KJ05 & 77 & LIGH I WATER REACTOR TECHNOLCGY \\
\hline $\mathrm{KGO3}$ & 77 & FAST FLUX TEST FACILITY (FFTF) & KJo6 & 77 & TECHNOLOGY DEVELOPNENT \& SPECIAL PROJECT \\
\hline $\mathrm{K} 504$ & 77 & TEST FACILITIES & $\mathrm{KJ} 07$ & 77 & SPACE APPL ICATIONS \\
\hline kroj 5 & 77 & SAFETY & KJ08 & 77 & ADVANCED ISOTOPE SEPARATIGN TECHNOLOGY \\
\hline KGO6 & 77 & ENGINEERED SYSTEMS AND COMPONENTS & KJo9 & 77 & NUCLEAR ENERGY ASSESSMENTS \\
\hline$K G 07$ & 77 & PHYSICS & KKOn & 78 & MULTI-RESOURCE \\
\hline KGO8 & 77 & NATERIALS & KKOL 1 & 78 & POLICY AND PROGRAM ANALYSIS \\
\hline KGO9 & 77 & FUELS & $\mathrm{KKO2}$ & 78 & GENERAL SYSTEMS STUDIES \\
\hline KG11 & 77 & REACTOR. CORE COMPONENTS & KNOO & 78 & OTHER \\
\hline K.HOO & 77 & NAVAL R.EACTOR DEVELGPMENT & KNO! & 78 & PR.GGRAM DIRECTION - MANAGEMENT SUP \\
\hline
\end{tabular}




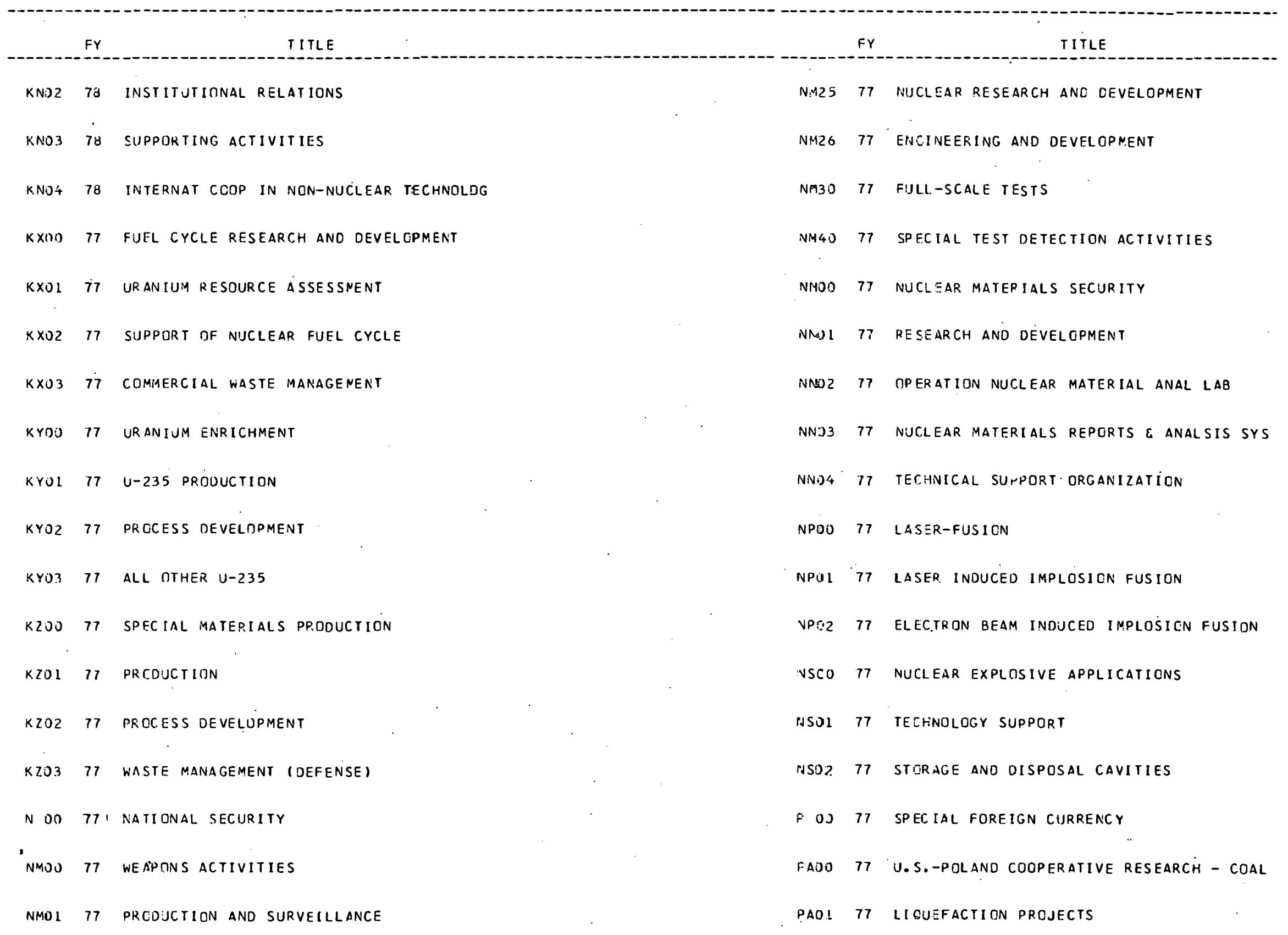




\begin{tabular}{|c|c|c|c|c|c|}
\hline & FY & TITLE & & FY & TITLE \\
\hline PAO2 & 77 & MAGNETOHYORODYAMICS (MHC) & RW02 & 77 & U MILL TAILINGS REMEDIAL ACTION PROGRAM \\
\hline PAO3 & 77 & ADMINISTRATIVE COST & RW03 & 77 & SAFETY STUDIES $\&$ OEVEL CPER GUIDELINES \\
\hline 8 un & 77 & ENUIRONMENTAL \& SAFETY FESEARCH & RYOJ & 77 & REACTOR SAFETY FACILITIES \\
\hline RTUOO & 77 & BICMEOICAL \& ENVIRONMENTAL RESEARCH & RYOl & 77 & LOSS OF FLJID TEST (LOFT) \\
\hline RTOL & 77 & HEDLTH STUDIES & RYO2 & 77 & PLENUM FILL EXPFRIMENTAL FACILITY (PFE) \\
\hline RTU2 & 77 & BIOLOGICAL STUDIES & U OU & 77 & PRCIGRAM SUPPORT \\
\hline RTJ3 & 77 & ENVIRONMENTAL STUDIES & UAOU & 77 & PRCGRAM DIRECTION \\
\hline RTu 4 & 77 & PHYSICAL AND TECHNOLOGICAL STUDIES & UALI & 77 & PERSONAL SERVICES \\
\hline pros & 77 & ANALYSIS AND ASSESSMENT & UAL 2 & 77 & PERSCNNEL BENEFITS \\
\hline ET06 & 77 & EDLCATION AND TRAINING & UA13 & 77 & BENEFITS FOR FORMER PER SGNNEL \\
\hline R.uou & 77 & ENVIRONMENTAL CONTRCL TECHNOLOGY & JA2J & 77 & CONTRACTUAL SERVICES $\varepsilon$ SUPPLIES \\
\hline RUD I & 77 & FOSSIL ENERGY & UA21 & 77 & TRAVEL \& TRANSPORTATION OF PERSONS \\
\hline RUit2 & 77 & SOLAR GEOTHERMAL \& ENERGY CGNSERVATION & UA40 & 77 & TAXES \& INDEMNITIES \\
\hline RUO3 & 77 & NJCLEAR ENERGY & JBOO & 77 & COMMUNITY OPERATIONS \\
\hline RUN04 & 77 & MGMT OF SURPLUS CONTAMINATED FACILITIES & UBO & 77 & MUNICIPAL SERVICES \\
\hline RUO5 & 77 & TRA.VSP ORTATIDN & UBO 7 & 77 & COMMUNITY ASSISTANCE \\
\hline RwOO & 77 & CPERATIONAL SAFETY & UCOO & 77 & SECJRITY INVESTIGATIONS \\
\hline RWOL & 77 & AERIAL RAUIOLOGICAL MONITCRING SYS(ARMS) & UC.OI & 77 & FED $: R A L$ BUTREAU OF INVESTIGATION \\
\hline
\end{tabular}




\begin{tabular}{|c|c|c|c|c|}
\hline & FY & TITLE & FY & TITLE \\
\hline UCO2 & 77 & CIVIL SERVICE COMMISSION & 77 & MANPOWER INFORMATIGN \\
\hline j000 & 77 & INFORMATION SERVICES & 77 & GENERAL TECHNOLOGY SUPPCRT \\
\hline JDúl & 77 & PUBLIC AWARENESS & 77 & TECHNOLDGY SUPPORT \\
\hline U002 & 77 & TECHNICAL INFORMATION SERVICES & 77 & COST OF WORK FOR OTHERS \\
\hline U003 & 77 & MAJOR INTERNATIONAL CONFERENCES & 78 & COST OF WORK FOR OTHERS \\
\hline UEOS & 77 & EEO CUNTRACT COMPLIANCE & 77 & COST OF WORK FOR OTHERS \\
\hline UE 11 & 77 & PERSONAL SERVICES & 77 & COST OF PRODUCTS SOLD \\
\hline UEL 2 & 77 & PERSONNEL BENEFITS & 87 & COST OF SERVICES PERFORNED \\
\hline UE 13 & 77 & BENEFITS FOR FORMER PERSONNEL & 77 & COST OF UEA SERVICES \\
\hline UE20 & 77 & CONIRACTUAL SERVICES $\varepsilon$ SUPPLIES & 77 & COST OF SEPARATIVE WORK - TOLL ENRICHING \\
\hline UE21 & 77 & TRAVEL $\varepsilon$ TRANSPORTATICN OF PERSONS & 77 & COST DF SS MATERIAL SOLD \\
\hline UFOO & $? 7$ & GENERAL SYSTEMS STUDIES & 77 & COST OTHER. SPECIAL PRODUCTS SOLO \\
\hline UFO 1 & 77 & SYSTEMS STUDIES AND ANALYSES & 77 & COST OF RECOVERY OF SS MATERIAL \\
\hline UGOn & 77 & GENERAL TECHNOLOGY TRANSFERS & 78 & COST OF WORK FOR OTHERS \\
\hline UGO1 & 77 & RESEARCH \& DEVELOPMENT COMMERCIALIZATION & 78 & COST OF PRODUTS SOLD \\
\hline UGO2 & 77 & TECHNOLOGY UTILIZATION & 78 & COST OF SERVICES PERFORNED \\
\hline UG03 & 77 & ENERGY INVENTION EVALUATION & 78 & COST OF UESA SERVICES \\
\hline UHOO & 77 & MANPOWER DEVELOPMENT & 78 & COST OF SEPARATIVE WORK - TOLL EN \\
\hline
\end{tabular}




\begin{tabular}{|c|c|c|c|c|c|}
\hline & FY & TITLE & & FY & TITLE \\
\hline YA41 & 77 & DETAILS OF URANIUM ENRICHMENT DPR COSTS & $W N: 36$ & 78 & COST OF SS MATERIAL SILC \\
\hline$Y 450$ & 77 & CPERATING INDIRECT LABCF & WN 7 & 73 & COST OF OTHER SPECIAL PRODUCTS SELD \\
\hline YA55 & 77 & SUPPORT & WNOS & 78 & COST OF RECOVERY OF SS NATERIAL \\
\hline YAGO & 77 & WORK FOR ERDA CONTRACTORS - INIDENT IF I & WNI 7 & 78 & COST DF SALE OF PETROLEUM \\
\hline YA9, & 77 & NONRE I MBUR SABLE TRANSFERS & Y 00 & 77 & CTHER COSTS \& CREOITS \\
\hline YA95 & 77 & INTEREST ON GOVERNMENT INVESTMENT & & 78 & GTHER COSTS AND CREDITS \\
\hline YNOO & 78 & OIHER COSTS AND CREDITS & YADS & 77 & OTHER COSTS \& CREDITS \\
\hline YNOL & 78 & UNOISTRIBUTED COST & YAOL & 77 & UNOISTRIBUTED COSTS \\
\hline YNO? & 78 & AJUSTMENT PRIOR YRS COST CF OPERATIONS & YAD? & 77 & ADJUSTMENT PRIOR YRS COST OF GPERATIONS \\
\hline YNO3 & 78 & PLANT ANO EQUIPMENT ADJUSTMENTS & YAD3 & 77 & PLANT \& EQUIPMENT ADJUSTMENTS \\
\hline YNO4 & 78 & CONATIONS COMMIJNITY PROPERTY & YAO' & 77 & DONATIONS OF COMMUNITY FROPERTY \\
\hline YNO7 & 78 & DISTRIBUTION OF RADIOSOTOPES WITHIN DOE & YAOT & 77 & DIST OF RAOIOISOTOPES WITHIN ERDA \\
\hline YNOB & 78 & DISTRIBUT OF STABLE ISOTOPES WITHIN DOE & YAOB & 77 & DIST OF STABLE ISOTOPES HITHIN ERDA \\
\hline YNUS & 78 & MI SCELLANEOUS GTHER COSTS - NONFUND & YAO9 & 77 & MISCELLANEOIJS OTHER COSTS - NCNFUNO \\
\hline YNLO & 78 & EXTRA OROINARY ADJUSTMENTS-CURRENT YEAR & YALO & 77 & EXTRA OROINARY AOJUSTMENTS-CURRENT YEAR \\
\hline YN21 & 78 & TRAVEL DUTSIDE THE UNITED STATES & YA21 & 77 & TRAVEL OUTSIDE THE UNITEO STATES \\
\hline YN31 & 78 & DOE PERSONAL SERVICES CCSTS $\&$ DIST & $Y A 31$ & 77 & ERDA PERSONAL SERVICES COSTS $\varepsilon$ OIST \\
\hline YN32 & 78 & DOE CJST OF TRAVEL SUBJ TO TRA REG \& DIS & YA32 & 77 & ERDA COST TRAVEL SURJ TRAVEL REG \& DIST \\
\hline
\end{tabular}




\begin{tabular}{|c|c|c|c|c|c|}
\hline & FY & TITLE & & FY & TI ILE \\
\hline Y N4 1 & 70 & DETAILS OF URANIUM ENRICHMENT DPER COSTS & $2 A 19$ & 77 & CTHER REVENUE \\
\hline YN50 & 78 & CPERATING INDIRECT LABCR & ZNDO & 78 & REVENUES \\
\hline YN55 & 73 & SUPPURT & éNOL & 73 & REVENUE FKOM PRGDUCTS SCLO \\
\hline YNGO & 78 & WORK FOR DOE CCNTRACTCRS - INCENTIFIED & ¿NO2 & 73 & REVENUE FROM SERVICES \\
\hline Y.N90 & 79 & NDNREI MBUR SABLE TRANSFERS & ¿NO3 3 & 78 & REVENUE FROM JUESA SERVICES \\
\hline Y.N95 & 78 & INTEREST ON GOVERNMENT INVESTMENT & $2 . \mathrm{No} 4$ & 18 & REV FM SCH ADJ RELAT AFC TOLL ENRICH CCN \\
\hline 2 1): & 77 & REVENUES APPLIED & 2 No5 5 & 13 & FEV RELATED SALES CF SEP WORK - TOLL ENR \\
\hline & $7 d$ & REVENUES & ZNNO & 78 & REVFNUE FROM SALE OF SS MATERIALS \\
\hline ZAJ: & 77 & REVENIJES APPLIED & 2 NOT 7 & 78 & REV FROM SALES OF OTHER SPECIAL PRODUCTS \\
\hline $2 A 01$ & .77 & REVENUE FROM PRDDUCTS SCLD & $\therefore$ No8 & 78 & REVENUE FROM LEASE GF MATERIALS \\
\hline $2 A() 2$ & 77 & RE.VEN!JE FRGM SERVICES & ZNoG & 73 & REVENUE FROM RECOVERY OF SS MATERIAL \\
\hline $2 A D 3$ & 77 & REVENUE FROM UEA SERVICES & ENLS & 78 & REVENUE (INTEREST) FROM DEFERRED SALES \\
\hline $2 \Delta .75$ & 77 & REV RELATED SALES SEP KCRK-TOLL EARICH & ZNI I & 73 & REV FM TERMINATIONS TOLL ENRICH CCNTRACT \\
\hline ZADG & 77 & REVENUE FROM SALE OF SS MATERIALS & $? N 16$ & 78 & REV FM OIL ROYALTIES ON LEASED LAND \\
\hline $2 A) T$ & 77 & REV FM SALES OF CTHER SPECIAL PROCUCTS & ?NI 7 & 78 & REVENJE FROM SALE GF PETRCLEUM \\
\hline ZAOB & 77 & REVENUE FRCM LEASE CF MATERIALS & ZNN 8 & 78 & REV FM SALE \& TRANSMISSION ELECTRIC ENG \\
\hline 22409 & 77 & REVENUE FRDM RECOVERY OF SS MATERIALS & ZN19 & 78 & OTHER REVENUE \\
\hline $\mathrm{ZAL} \mathrm{i}$ & 77 & REVENUE (INTEREST) FROM DEFERRED SALES & $35 \mathrm{~A}$ & 78 & ENERGY SUPPLY - RES ANO TECHNO $\cdots$ DEVEL \\
\hline
\end{tabular}




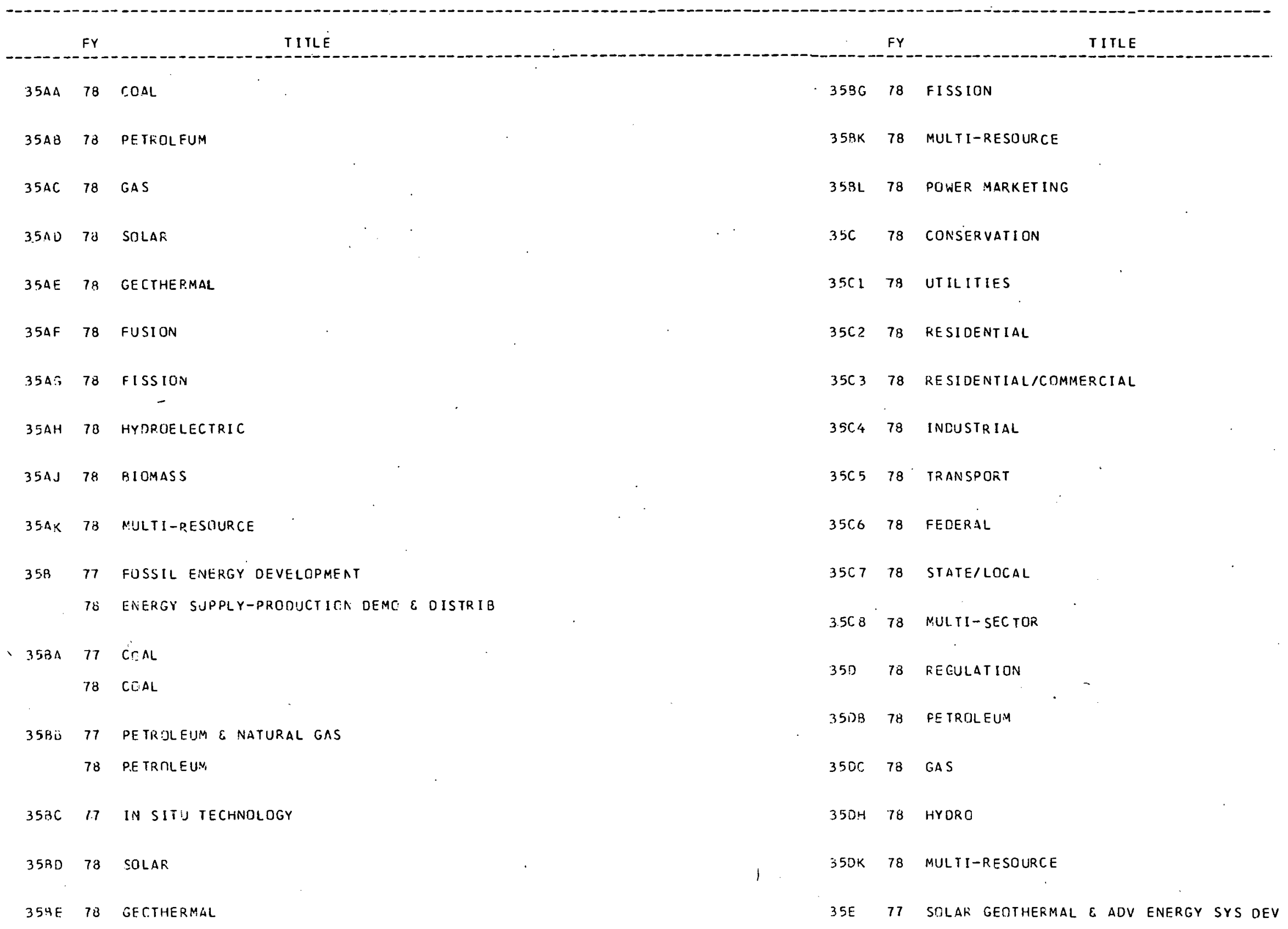




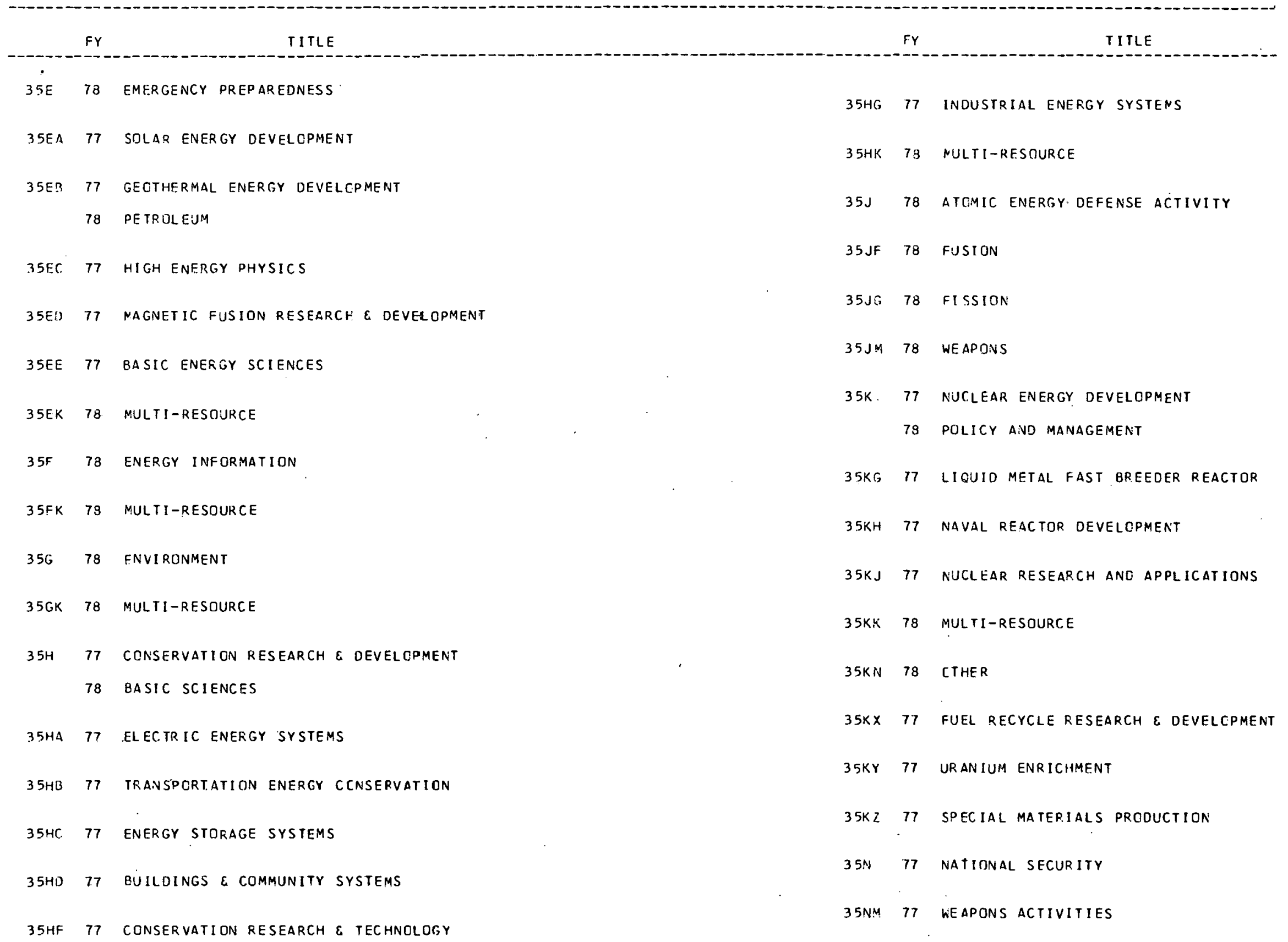




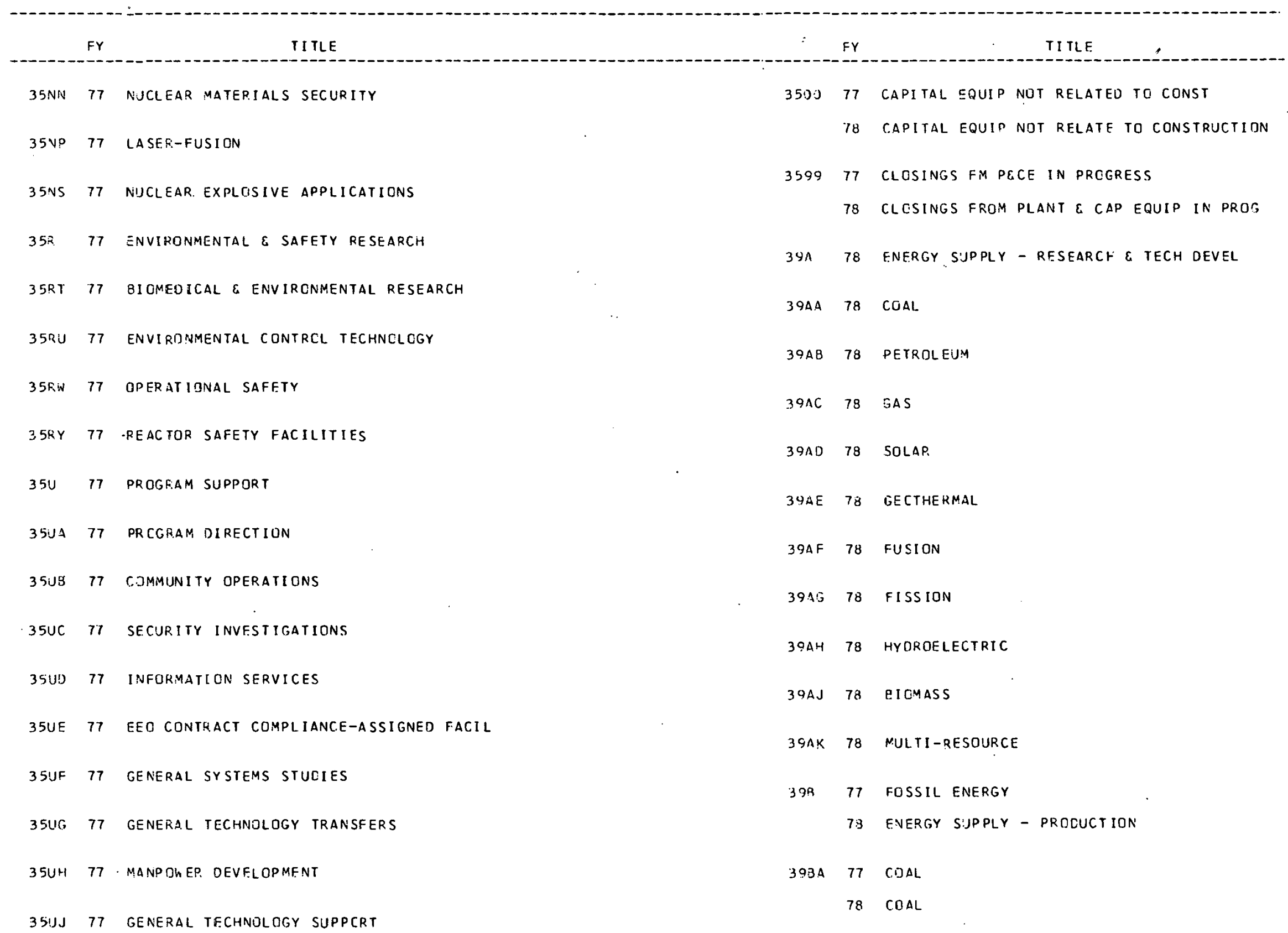




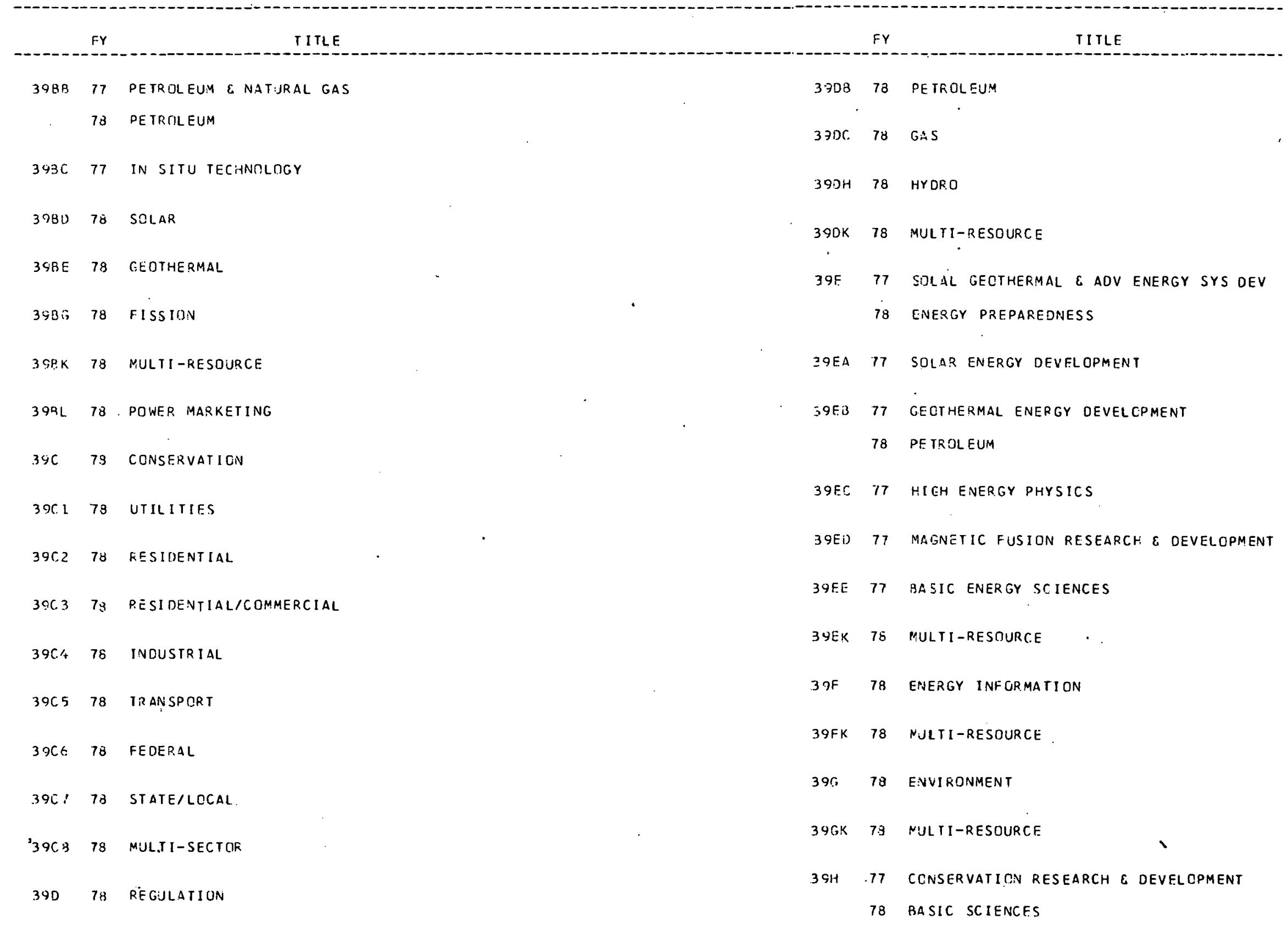




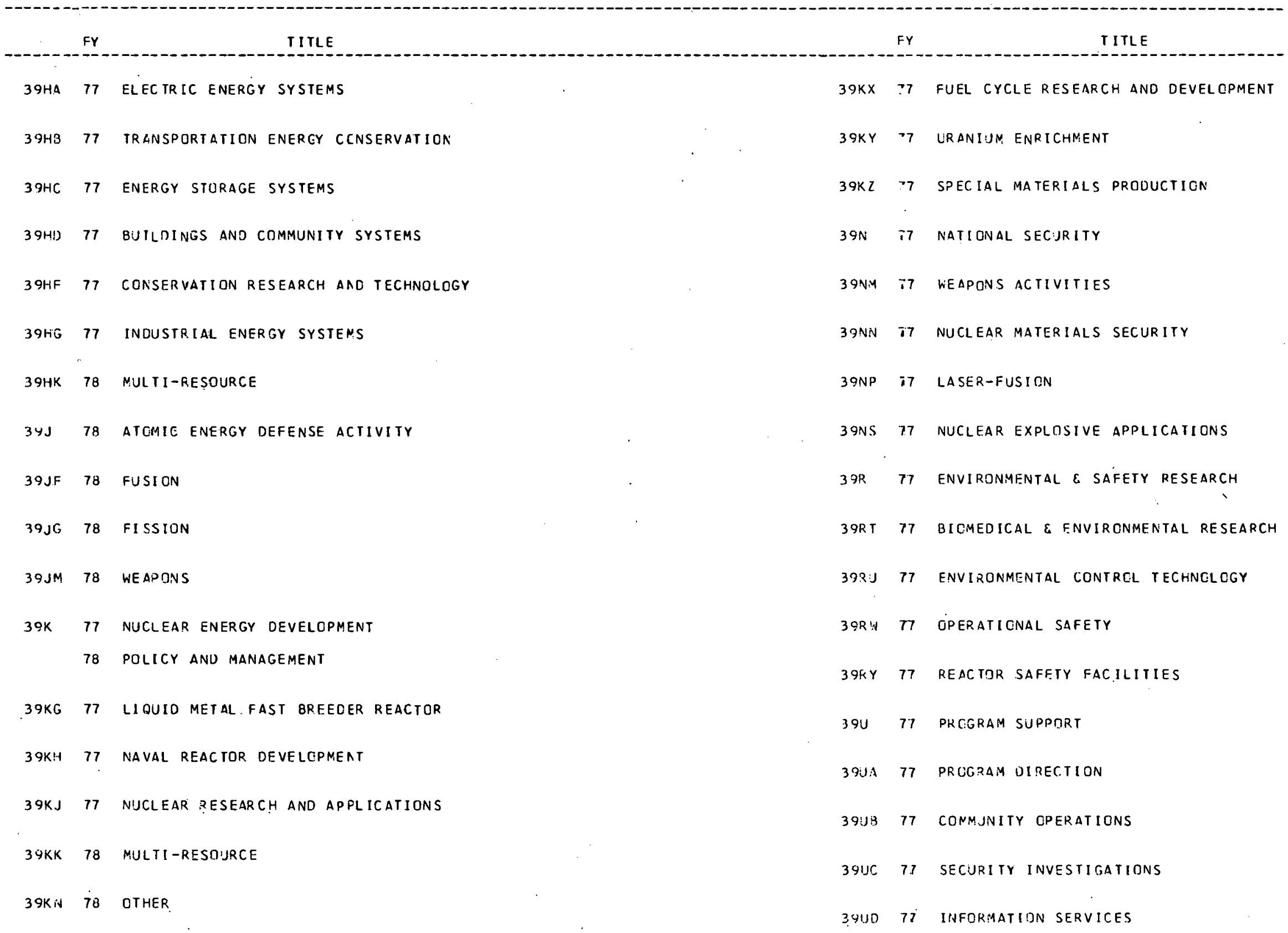




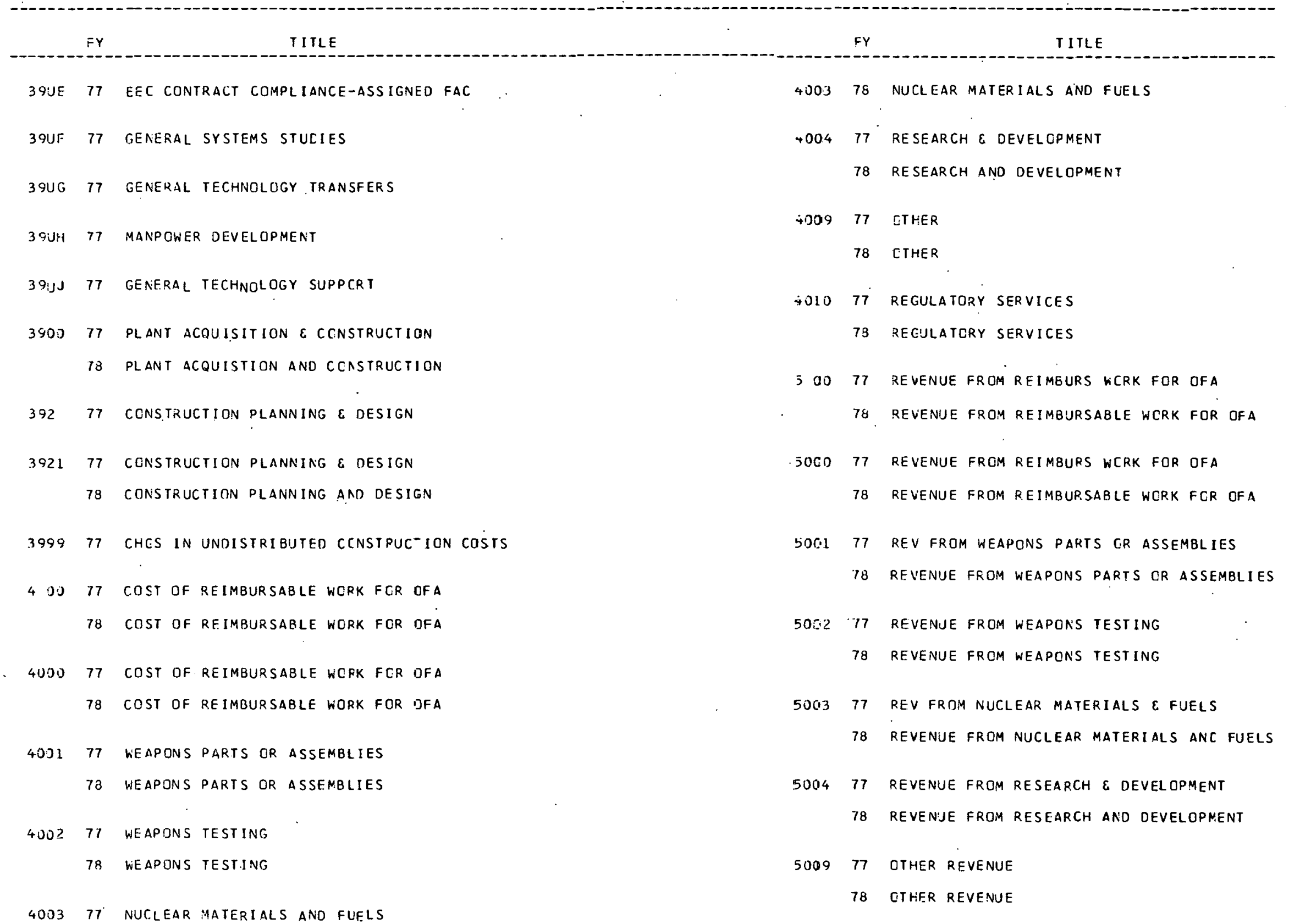




\begin{tabular}{|c|c|c|c|c|c|}
\hline & FY & TITLE & & $F Y$ & II TLE \\
\hline 5010 & 77 & $\begin{array}{l}\text { REVEN:JE FROM REGULATORY SERVICES } \\
\text { CEVENUE FRGM REGULATORY SERVICE }\end{array}$ & U1KN & 78 & OTHER \\
\hline 800 & $\begin{array}{l}77 \\
78\end{array}$ & $\begin{array}{l}\text { DUMMY TITLE } \\
\text { RECONCILING ITEMS }\end{array}$ & 8100 & 77 & $\begin{array}{l}\text { CHANGES IN INVENTORIES } \varepsilon \text { COLLATERAL FUND } \\
\text { CHANGE IN INVENTORIES }\end{array}$ \\
\hline $81 \mathrm{~A}$ & 78 & ENERGY SUPPLY-RESEAR.CH \& TECHNOLGGY DEV & 8101 & 77 & INVENTORY CHANGES \\
\hline BlAF & 78 & FUSION & 8102 & 77 & CHANGES IN COLL FUNOS \& OTHER DEPOSITS \\
\hline SLAG & 78 & $F I \subseteq S I J N$ & 8140 & $7 \overline{3}$ & REIMBURSABLE WORK \\
\hline AlAK & 78 & MULTI-RESOURCE & 8201 & 78 & $\begin{array}{l}\text { RECONC ILING TRANSFERS \& OTHER RECIP ITEM } \\
\text { RECONC ILING TKANSFER \& OTHER RECIP ITEMS }\end{array}$ \\
\hline $81 B$ & 78 & ENERGY SIJPPLY-PFODUCTIOA DEMO \& DISTRIB & 8201 & $7 i$ & INTERFINANCIAL PLAN TRANSFERS \\
\hline BIBG & 78 & $F I \subseteq S I O N$ & & 78 & INTERF.INANCIAL PLAN TRANSFERS \\
\hline $81 G$ & 78 & ENVI RONMENT & $82.0 ?$ & 77 & INTRAF INANCIAL PLAN TRANSFERS \\
\hline 31GK & 78 & MULTI-RESOURCE & & 78 & INTRAF INANCIAL PLAN TRANSFERS \\
\hline $81 \mathrm{H}$ & 78 & BASIC SCIENCES & 8203 & $\begin{array}{l}77 \\
78\end{array}$ & $\begin{array}{l}\text { ADVANCFS TO INTEGRATEO CONTRACTORS } \\
\text { ADVANCES TO INTEGRATED CONTRACTORS }\end{array}$ \\
\hline $31 \mathrm{HK}$ & 73 & MULTI-RESOURCE & 8300 & 77 & CHANGES IN UNDELIVEREO CRDERS \\
\hline $31 \mathrm{~J}$ & 73 & ATOMIC ENERGY DEFENSE ACTIVITY & & 78 & CHANGES IN UNDELIVEREC CRDERS \\
\hline $81 \mathrm{JF}$ & 78 & FUSION & 8301 & 77 & $\begin{array}{l}\text { OBLIGATIONS INCURRED } \\
\text { OBLIGATIONS INCURREC }\end{array}$ \\
\hline $81 \mathrm{~J}$ & 78 & WEAPDNS & 8302 & 77 & $\begin{array}{l}\text { PAYMENTS } \\
\text { PAYMENTS }\end{array}$ \\
\hline
\end{tabular}




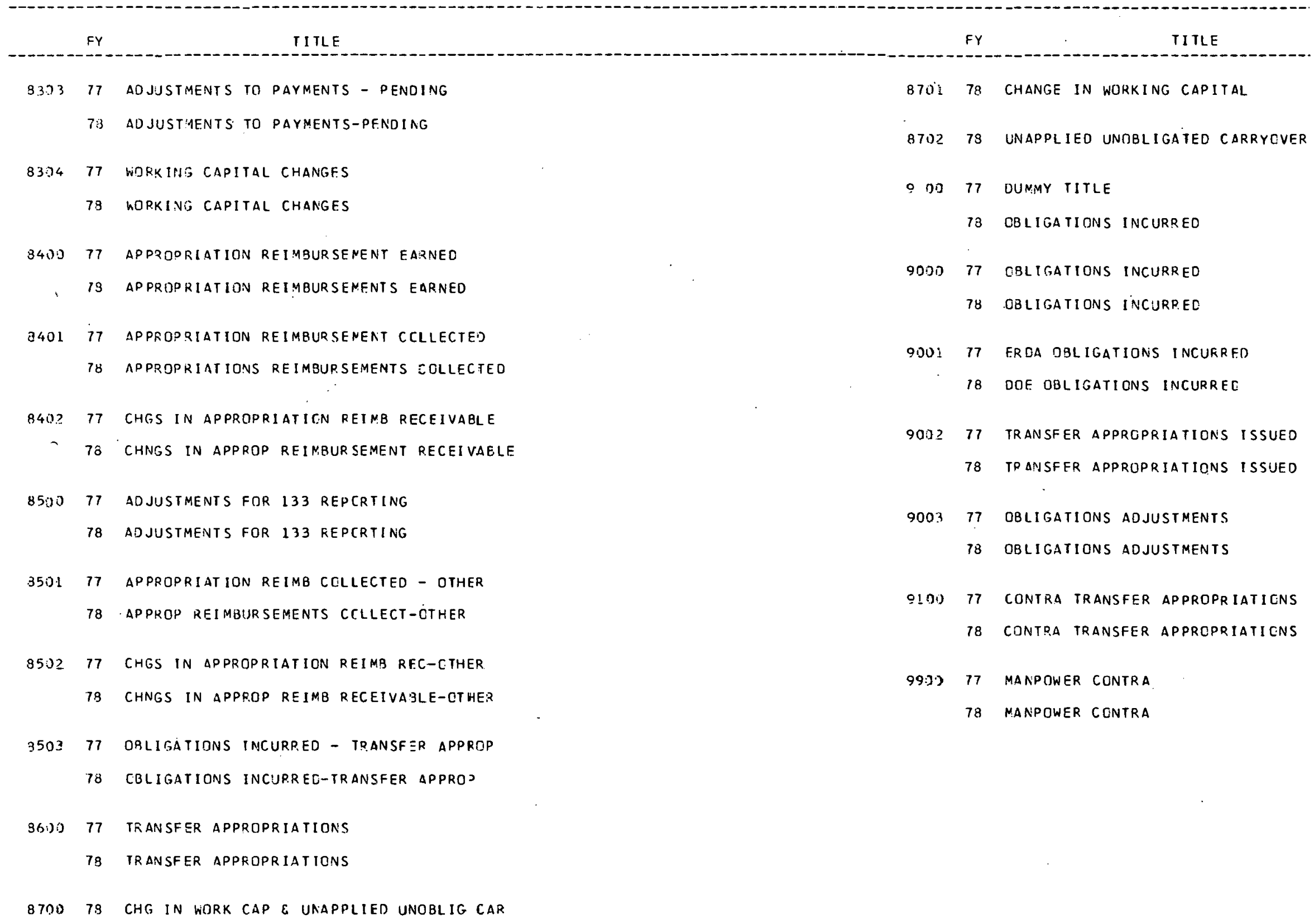


Organizational Code DOE Office

AL8-3400 Laramie Energy Research Center

AL9-3100 Albuquerque Operations Office

AP1-7070 Alaska Power Administration

BM2-6051 Bureau of Mines, Pittsburgh

BM3-6052 Bureau of Mines, Denver

BPA-7059 BPA--Pasco Maint.--Walla Walla

BPB-7060 BPA--Maint.--Wenatchee

BPC-7061 BPA--Ross Whse--Whse Unity

BPD-7062 BPA--Library

BP1-7050 BPA--Materials and

Procurement Branch

BP2-7051 BPA--Branch of Land

BP3-7052 BPA--Contracts Managers Office

BP4-7053 BPA--Customer Service

BP5-7054 BPA--Transmission Constr.

BP6-7055 BPA--Substation Constr.

BP7-7056 BPA--Ross Whse--Parts Rm.

BP8-7057 BPA--Covington Whse.--Seattle

BP9-7058 BPA--Bell Substation--Spokane

BR1-6070 Western Area Power Admin.

CH6-4600 Pittsburgh Energy Research Center

CH7-4900 Grand Forks Energy Research Center

CH8-4800 Bartlesville Energy Research Center

CH9-4200 Chicago Operations Office

CR9-4300 Clinch River Breeder Reactor Office

FRA-6040 Federal Region X Office, Seattle

FR1-6041 Federal Region I Office, Boston
Organization Code

DOE Office

FR2-6042 Federal Region II Office, New York City

FR3-6043 Federal Region III Office, Philadelphia

FR4-6044 Federal Region IV Office, Chicago

FR5-6045 Federal Region V Office, Atlanta

FR6-6046 Federal Region VI Office, Dallas

FR7-6047 Federal Region VII Office, Kansas City

FR8-6048 Federal Region VIII Office, Denver

FR9-6049 Federal Region IX Office, San Francisco

GJ9-1100 Grand Junction Office

ID9-4100 Idaho Operations Office

INA2-8020 Petroleum Reserves--Wyoming

NA3-8030 Petroleum Reserves--California

NV9-3300 Nevada Operations Office

OR8-2800 Morgantown Energy Research Center

OR9-2400 Oak Ridge Operations Office

PN9-4400 Pittsburgh Naval Reactors Office

RL9-2300

SE1-7010

SF9-3700

SR9-2600

ST9-4500

SW1-7030

WA2-9902

WA9-9900
Richland Operations Office

Southeastern Power Administration

San Francisco Operations Office

Savannah River Operations Office

Schenectady Naval Reactors Office Southwestern Power Administration

Procurement Operations, $\mathrm{HQ}$

All Other Offices - HQ 
PROCUREMENT AND FINANCIAL ASSISTANCE AWARD CODES

\section{Type Of Work Codes/Type Of Contract Codes}

Type Of Work Code

$\begin{array}{ll}\text { A-E } & \text { Architect Engineer } \\ \text { CONST } & \text { Onsite Construction } \\ \text { R\&D } & \text { Research \& Development } \\ \text { MSE-C } & \text { Materials, Supplies \& Equipment For Construction } \\ \text { MSE-O } & \text { Materials, Supplies \& Equipment For Other Than Construction } \\ \text { R\&US } & \text { Rent \& Utility Services } \\ \text { OPR } & \text { Operating Contract } \\ \text { OTHER } & \text { Other Services } \\ \text { OPGM } & \text { Other Programmatic }\end{array}$

\begin{tabular}{ll} 
Code & Fixed Price Types \\
\hline FFP & Firm Fixed-Price \\
FPE & Fixed.Price With Escalation \\
FPI & Fixed-Price With Incentive \\
FPR & Fixed-Price, Redeterminable
\end{tabular}

\section{Cost Types}

$\begin{array}{ll}\text { CNF } & \text { Cost, No Fee } \\ \text { CPIF } & \text { Cost-Plus-Incentive-Fee } \\ \text { CPFF } & \text { Cost-Plus-A-Fixed-Fee } \\ \text { CPAF } & \text { Cost-Plus-Award-Fee } \\ \text { T\&M } & \text { Time \& Materials } \\ \text { LH } & \text { Labor-Hour } \\ \text { COST-SHR } & \text { Cost-Sharing }\end{array}$

\section{Other Contractual Codes}

SRSA . Special Research Support Agreements

GRANT Grants

NF No Fund

COOP AGR Cooperative Agreements

\section{PKI-TOPA}

Prime Task Order or Project Agreement Number (PRI-TOPA) is part of the contract number. This number identifies separate distinct tasks under a contract. 
JCUREMENT AND FINANCIAL ASSISTANCE AWARD CODES

\section{Type Of Work}

\section{Code}

\begin{tabular}{ll}
\hline 1 & Architect-Engineer \\
$\mathbf{2}$ & Onsite Construction \\
$\mathbf{3}$ & Research \& Development \\
$\mathbf{4}$ & Materials, Supplies \& Equipment For Construction \\
$\mathbf{5}$ & Materials, Supplies \& Equipment For Other Than Construction \\
$\mathbf{6}$ & Rents \& Utility Services \\
7 & Operating Contract \\
$\mathbf{8}$ & Other Servires \\
$\mathbf{9}$ & Other Programmatic
\end{tabular}

\section{Type Of Procurement}

\section{Code}

1

2

3

4

5

6

7

8

9
Intracompany Or Interdepartmental

GSA Federal Supply Schedule

GSA Local Service Contract

Other Government Sources

Advertised

Negotiated Competitive

Negotiated Other

Unsolicited Prupusal

Sole Source

\begin{tabular}{ll} 
Code & Fixed-Price Types \\
\hline F & Firm Fixed-Price \\
E & Fixed-Price With Escalation \\
W & Fixed-Price With Incentive \\
R & Fixed-Price, Redeterminable
\end{tabular}

\section{Cost Types}

C

I

$P$

A

T

H

B
Cost, No Fee

Cost-Plus-Incentive-Fee

Cost-Plus-A-Fixed-Fee

Cost-Plus-Award-Fee

Time \& Materials

Labor-Hour

Cost-Sharing 
Type Of Contract (Cont.)

Code Other Contractual Codes

S

G

N

U
Special Research Support Agreement (SRSA)

Grant

No Fund (No Commitment To Provide ERDA Funds)

Cooperative Agreenıents

\section{Source of Procurement}

\section{Code}

Government

\section{1}

12

DOE

Other Federal Agencies

State \& Local Government

14

Foreign Governments

\section{Commercial}

20

30

60

Small Business

Big Business

Foreign

\section{Nonprofit Organizations}

41

University Or College

42

State-Owned Or Operated Laboratory Or Institute

44

45

46

47

Nonprofit Research Foundation Or Laboratory

Hospital

Industrial Or Commercial Research Laboratory

Foreign \& All Other

Type Of Action

Code

$\begin{array}{ll}\text { L } & \text { Leller Contract } \\ \mathrm{D} & \text { Definitive Contract } \\ \mathrm{P} & \text { Purchase Urder } \\ \mathrm{I} & \text { Interagency Agreements }\end{array}$

\section{Contract Status}

\section{Code}

\section{0}

11

12

30

31

70

\author{
Active \\ Active - Extension In Process \\ Active - Terminal Period \\ Completed \\ Completed - Contingency For Warranty Or Claim Still Exists \\ Closed-Out
}

\section{PRI-TOPA}

Prime Task Order or Project Agreement Number (PRI-TOPA) is part of the contract , number. This number identifies separate distinct tasks under a contract. 


\section{United States}

\section{Department of Energy}

Washington, DC 20545

Postage and Fees Paid

U.S. Department of Energy

DOE-350

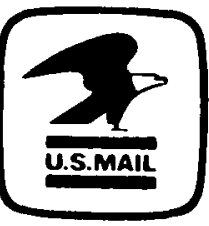

Official Business

Penalty for Private Use, $\$ 300$ 